University of Louisville

ThinkIR: The University of Louisville's Institutional Repository

\title{
The relationship between trauma and health-related quality of life in lung cancer patients : the potential protective role of mindfulness.
}

Rene Bayley-Veloso

University of Louisville

Follow this and additional works at: https://ir.library.louisville.edu/etd

Part of the Clinical Psychology Commons, and the Health Psychology Commons

\section{Recommended Citation}

Bayley-Veloso, Rene, "The relationship between trauma and health-related quality of life in lung cancer patients : the potential protective role of mindfulness." (2017). Electronic Theses and Dissertations. Paper 2761.

https://doi.org/10.18297/etd/2761

This Doctoral Dissertation is brought to you for free and open access by ThinkIR: The University of Louisville's Institutional Repository. It has been accepted for inclusion in Electronic Theses and Dissertations by an authorized administrator of ThinkIR: The University of Louisville's Institutional Repository. This title appears here courtesy of the author, who has retained all other copyrights. For more information, please contact thinkir@louisville.edu. 
THE RELATIONSHIP BETWEEN TRAUMA AND HEALTH-RELATED QUALITY OF LIFE IN LUNG CANCER PATIENTS:

THE POTENTIAL PROTECTIVE ROLE OF MINDFULNESS

\author{
By \\ René Bayley-Veloso, M.A. \\ B.A., University of Illinois at Chicago, 2012 \\ M.A., University of Louisville, 2014

\begin{abstract}
A Dissertation
Submitted to the Faculty of the

College of Arts and Sciences of the University of Louisville

in Partial Fulfillment of the Requirements

for the Degree of
\end{abstract}

Doctor of Philosophy in Clinical Psychology

Department of Psychological and Brain Sciences

University of Louisville

Louisville, Kentucky

August 2017 
Copyright 2016 by René Bayley-Veloso

All rights reserved 



\title{
THE RELATIONSHIP BETWEEN TRAUMA AND HEALTH-RELATED QUALITY OF LIFE IN LUNG CANCER PATIENTS: \\ THE POTENTIAL PROTECTIVE ROLE OF MINDFULNESS
}

\author{
By \\ René Bayley-Veloso, M.A. \\ B.A., University of Illinois at Chicago, 2012 \\ M.A., University of Louisville, 2014 \\ A Dissertation Approved on
}

June 30, 2016

By the following Dissertation Committee:

Dissertation Director Dr. Sandra Sephton

Dr. Paul Salmon

Dr. Benjamin Mast

Dr. Elizabeth Cash

Dr. Tamara Newton 


\section{DEDICATION}

This dissertation is dedicated to my mother, Laura Bayley, whose love and support never ceased. It was her grace, compassion and unyielding strength during her cancer experience that served as the inspiration for this research 


\section{ACKNOWLEDGEMENTS}

This dissertation would not have been possible without the steadfast support and guidance of Dr. Sandra Sephton. Her unending support, both personally and professionally, has allowed me to find confidence in my voice as a researcher. I am extremely grateful for the co-mentorship of Dr. Paul Salmon, who invited me into his lab, and provided the foundation of my graduate training. I would not be the scientistpractitioner I am today if not for their expert mentorship. I am also sincerely grateful for the guidance and support from my friend and unofficial mentor, Dr. Elizabeth Cash, whose encouragement lifted my spirits when I needed it most. I would like to thank Drs. Tamara Newton and Benjamin Mast for providing helpful suggestions and serving on my dissertation committee. I would like to thank everyone in the Biobehavioral Research Laboratory, especially Lauren Zimmaro for her dedication to the study, Kala Phillips for her friendship and unwavering cheerfulness, and Chelsea Siwik for her unfaltering dependability and support. I would also like to express my sincere gratitude to my dear friend and colleague, Yvette Szabo, for her tireless support, encouragement and friendship over the last four years. Finally, I would like to thank my husband, Jan Veloso, my father, David Bayley Sr., my sister, Heather Bayley, my brother, David Bayley Jr., and my grandmother, Lucy Candela, for providing their unconditional love and support over the years that have allowed me to pursue my passion. Funding for this study was provided by the Commonwealth of Kentucky Lung Cancer Research Program. 


\title{
ABSTRACT \\ THE RELATIONSHIP BETWEEN TRAUMA AND HEALTH-RELATED QUALITY OF LIFE IN LUNG CANCER PATIENTS: \\ THE POTENTIAL PROTECTIVE ROLE OF MINDFULNESS
}

\author{
René Bayley-Veloso
}

June 30, 2016

Lung cancer is the most common malignant disease worldwide, and the rapid decline in functioning due to the often-later stage diagnosis can strongly impact a patient's health-related quality of life (HRQOL). Palliative care, with the aim of improving later-stage HRQOL, is often a main goal of treatment. Compared to other cancer types, patients with lung cancer experience the greatest amount of psychological distress during and after treatment. Prior trauma exposure, and the potentially traumatic nature of the cancer experience, can further complicate HRQOL. Mindfulness, an innate or acquired capacity for sustaining attention in the present moment with qualities of selfwarmth and compassion, may serve as a protective factor that promotes well-being following a cancer diagnosis. The present study investigated the associations of trauma, HRQOL and mindfulness in a sample of lung cancer patients. It also examined the potential moderating role dispositional mindfulness may take in buffering the relationship between trauma and HRQOL.

Forty-six participants diagnosed within the last five years with non-small cell lung cancer $(34$ females, 12 males, mean age $=61.5)$ were administered self-report assessments of trauma history, traumatic distress appraisal, cancer-specific distress, HRQOL and mindfulness. Hierarchical linear regression analyses were employed to investigate the relationships of interest. 
Primary analyses revealed that traumatic distress appraisal and cancer-specific distress were negatively associated with HRQOL. Mindfulness was negatively associated with cancer-specific distress and positively associated with HRQOL. The relationships between trauma (as measured by traumatic distress appraisal and cancer-specific distress) and HRQOL were not moderated by mindfulness.

The current study supports the notion that trauma factors engender a vulnerability to having poorer HRQOL and that mindfulness may serve as a protective factor in the psychological adjustment to lung cancer and can improve quality of life. Future studies should further investigate mindfulness as both an explanatory construct and an intervention target to improve HRQOL in lung cancer patients. 


\section{TABLE OF CONTENTS}

PAGE

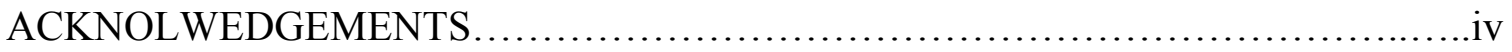

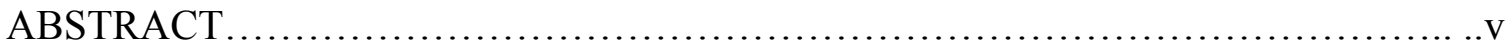

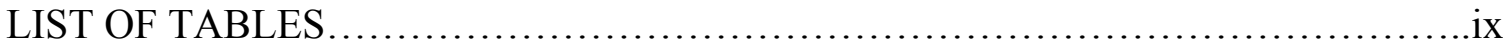

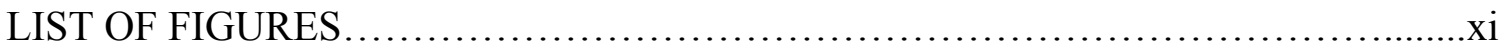

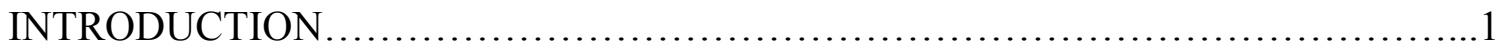

The Relationship Between Trauma and Cancer HRQOL..........................

Mindfulness.................................................................

The Relationship Between Trauma and Mindfulness..........................22

The Relationship Between Cancer HRQOL and Mindfulness.....................31

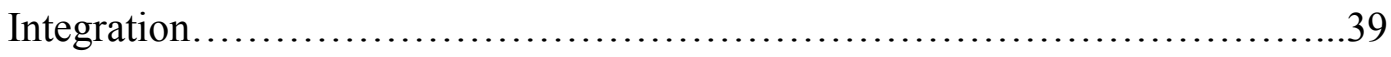

Focus of Current Study ................................................. 46

Summary and Hypotheses................................................47

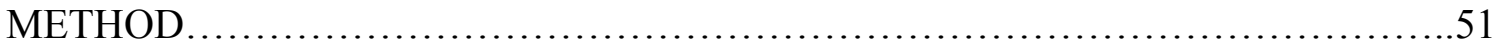

Recruitment............................................................ 51

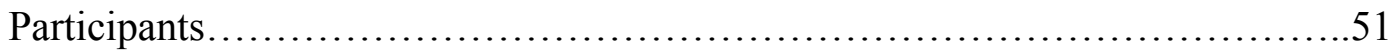

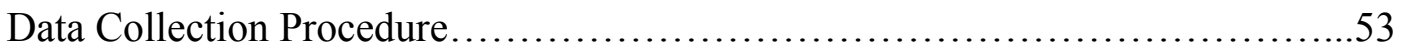

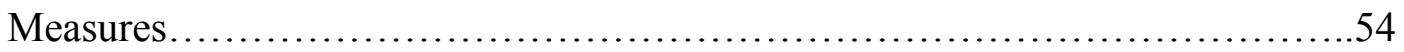

Statistical Analyses...................................................... 57

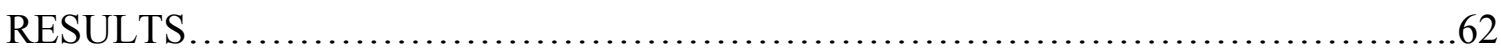

Sample Characteristics..................................................62

Preliminary Analyses....................................................67

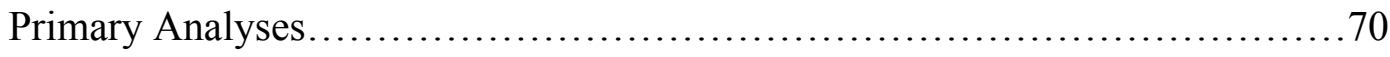

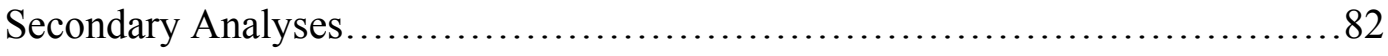


DISCUSSION ........................................................ 94

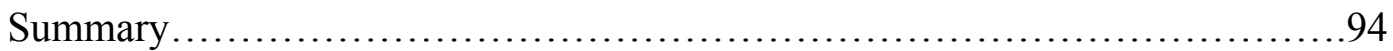

Main Findings..................................................... 95

Strengths and Limitations........................................... 108

Future Directions................................................... 111

Conclusions.....................................................113

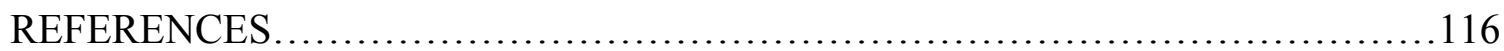

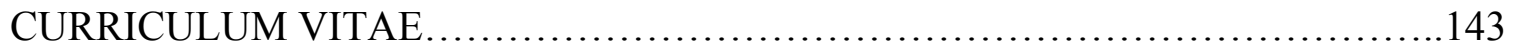




\section{LIST OF TABLES}

TABLE

PAGE

1. Demographic Characteristics of the Sample......................................61

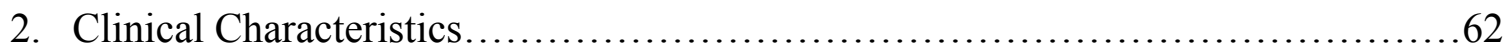

3. Mean and Standard Deviation for Study Variables..............................63

4. Bivariate Pearson Correlations Between Independent and Dependent

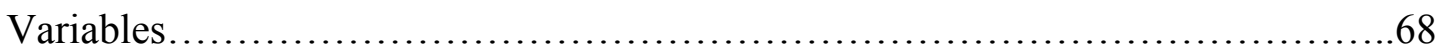

5. Bivariate Spearman Correlations Between Predictor, Control and Outcome

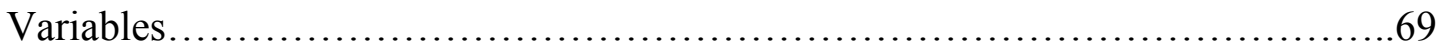

6. Summary of Hierarchical Regression Analyses Entering Frequency of Traumatic

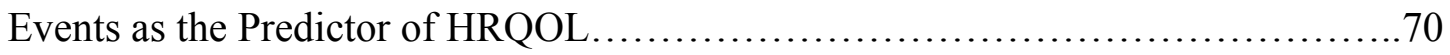

7. Summary of Hierarchical Regression Analyses Entering Traumatic Distress Appraisal

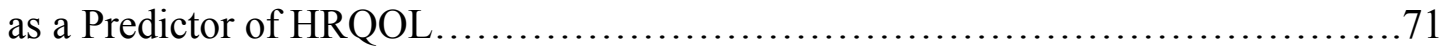

8. Summary of Hierarchical Regression Analyses Entering Cancer-Specific Distress as

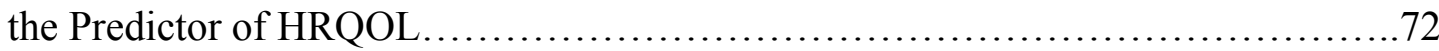

9. Summary of Hierarchical Regression Analyses Entering Mindfulness as the Predictor

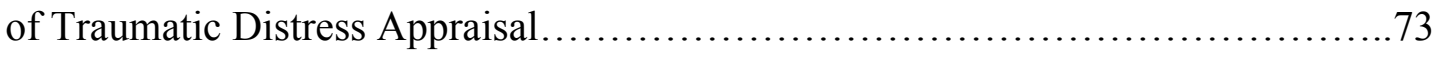

10. Summary of Hierarchical Regression Analyses Entering Mindfulness as the Predictor

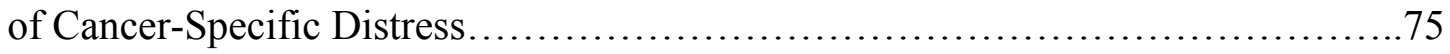


11. Summary of Hierarchical Regression Analyses Entering Mindfulness as the Predictor

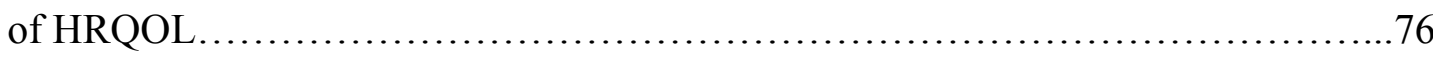

12. Summary of Hierarchical Regression Analyses Testing for Moderation of Mindfulness on Traumatic Distress Appraisal and HRQOL..........................79

13. Summary of Hierarchical Regression Analyses Testing for Moderation of Mindfulness on Traumatic Distress Appraisal on HRQOL with Three Control

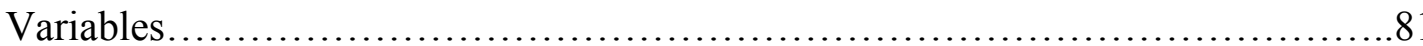

14. Bivariate Pearson Correlations Between Trauma Variables and Subscales of the

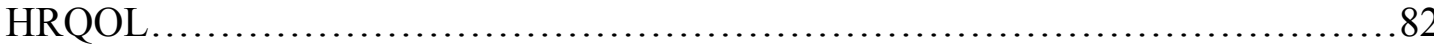

15. Bivariate Pearson Correlations Between the Subscales of the Cancer-Specific Distress

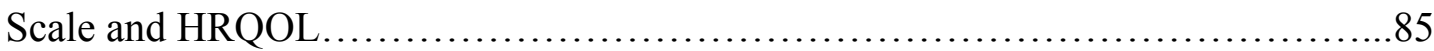

16. Bivariate Spearman Correlations Between the Subscales of the Cancer-Specific

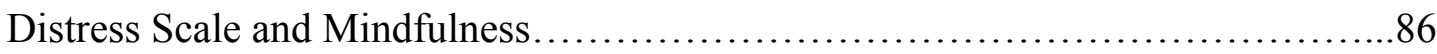

17. Bivariate Spearman Correlations Between the Subscales of Mindfulness and Cancer-

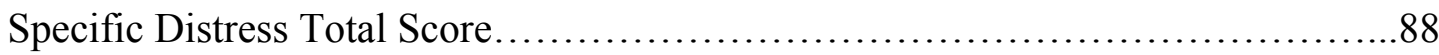

18. Bivariate Spearman Correlations Between Mindfulness and Specific Cancer

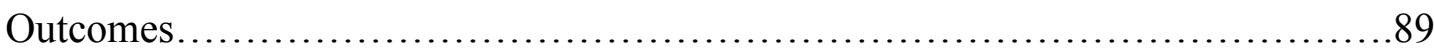

19. Bivariate Spearman Correlations Between the Subscales of Mindfulness and Total

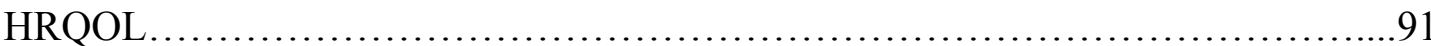




\section{LIST OF FIGURES}

FIGURE PAGE

1. Stress-Reducing Aspects of Mindfulness: Adaptation of the Transactional Model of

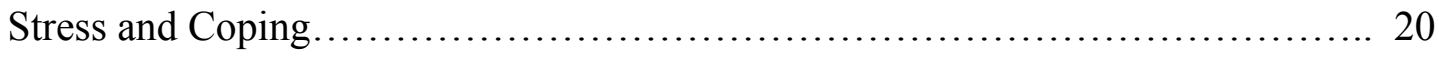

2. A Conceptual Model That Proposes the Potential Pathways of Intersection Between Variables of Interest..................................................48

3. Mindfulness moderates Traumatic Distress Appraisal on HRQOL .................49

4. Mindfulness Moderates Cancer-Specific Distress on HRQOL.......................50

5. Histogram of Clinical Cutoff Scores for the IES-R ..........................65

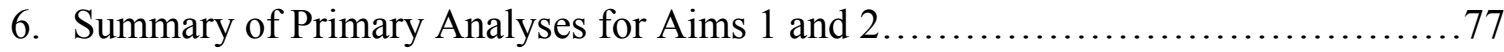

7. Associations Between Traumatic Distress Appraisal and Subscales of HRQOL.....83

8. Associations Between Cancer-Specific Distress and Subscales of HRQOL...........84

9. Associations Between subscales of Cancer-Specific Distress and Total HRQOL......86

10. Associations Between Total Mindfulness and Cancer-Specific Distress Subscales...87

11. Associations Between the Subscales of Mindfulness and Total Cancer-Specific

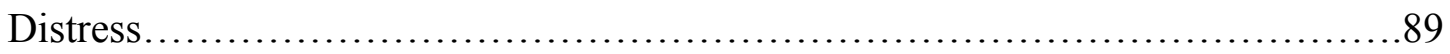

12. Associations Between Total Mindfulness and Subscales of HRQOL ................90

13. Associations Between Subscales of Mindfulness and Total HRQOL................92 


\section{INTRODUCTION}

For many, coping with cancer is marked by a sense of uncertainty, loss of control and constant change (Carlson, Speca, \& Segal, 2010), which complicates both adjustment to the diagnosis and perceived health-related quality of life (HRQOL). Due to frequently rapid decline, lung cancer patients experience high rates of anxiety, depression and distress compared to other cancer patients, which can negatively impact HRQOL (Carlsen, Jensen, Jacobsen, Krasnik, \& Johansen, 2005). Compared to other cancer types, patients with lung cancer experience the greatest amount of psychological distress during and after treatment (Cataldo, Slaughter, Jahan, Pongquan, \& Hwang, 2011). Prior trauma exposure, and the potentially traumatic nature of the cancer experience, can further complicate HRQOL (Fallah, Keshmir, Kashani, Azargashb, \& Akbari, 2012). Mindfulness, an innate or acquired capacity for sustaining attention in the present moment with qualities of self-warmth and compassion (Gilbert \& Procter, 2006), may serve as a protective factor that promotes well-being following a cancer diagnosis.

Lung cancer is the most common malignant disease worldwide, and the rapid decline in functioning due to the often-later stage diagnosis can strongly impact a patient's HRQOL. Palliative care, with the aim of improving later-stage HRQOL, is often a main goal of treatment (Carlsen et al., 2005). Common themes expressed by those receiving a diagnosis of lung cancer include an experience of uncertainty, experience of hope, reaching out for support, thoughts of death, feelings of shame and guilt, and 
concern for next of kin reactions (Berterö, Vanhanen, \& Appelin, 2008). Stigma associated with the diagnosis of lung cancer is commonly experienced by patients, regardless of smoking history, and often leads to internal causal attributions and blame that increase distress (Cataldo et al., 2011). The unique challenges presented by the experience of lung cancer compound the already stressful experience of cancer treatment (Berterö et al., 2008).

This dissertation reviews the relevant literature and explores how these three correlates of stress and coping - trauma, HRQOL and mindfulness - may be related to one another. Although relationships between each pair of variables have been investigated separately, a unified view of their inter-relationships has yet to be formulated. This paper proposes two theoretically derived models examining how these three constructs may be related within the context of lung cancer. The intention is to explain how mindfulness may influence both trauma and HRQOL by exploring: (1) the relationship between trauma and lung cancer HRQOL; (2) the relationship between trauma and mindfulness; and (3) the relationship between lung cancer HRQOL and mindfulness. With this as a foundation, the potential mechanisms through which mindfulness may promote resilience within the context of both trauma and lung cancer HRQOL are discussed, and one model is tested.

HRQOL is conceptualized as the extent that one's expected physical, emotional and social well-being are affected by a medical condition or treatment (Khanna \& Tsevat, 2007). HRQOL is increasingly being used as a primary outcome measurement in studies that evaluate the effectiveness and impact of cancer treatments (Guyatt, Feeny, \& Patrick, 1993). HRQOL can be used to estimate the burden of illness, as an end-point to clinical 
trials, to monitor clinical practice, and to help individuals track their own health utility to aid in making treatment decisions (Khanna \& Tsevat, 2007). Many factors influence HRQOL for cancer patients, including illness-related variables such as stage, cancer type and treatment (Wilson \& Cleary, 1995), and psychosocial variables such as coping, mental health status, and trauma history (Kangas, Henry, \& Bryant, 2002). The field of psycho-oncology now recognizes the importance of HRQOL as a main objective for psychosocial treatments, given that it has been shown to impact physical and mental health outcomes for patients (Casso, Buist, \& Taplin, 2004). As therapeutic options for patients with advanced lung cancer are largely palliative, improving HRQOL is considered an essential aspect of treatment (Camps, del Pozo, Blasco, Blasco, \& Sirera, 2009).

The cancer experience, and HRQOL, can be complicated by the possible presence of traumatic events in one's history that can impact coping with the stress of the disease (Green et al., 2000). This may be due to the potentially traumatic nature of the cancer experience that exacerbates distress symptoms caused by prior trauma. Trauma is conceptualized as experiencing, witnessing or learning about an event that involves actual or threatened death or injury (Gurevich, Devins, \& Rodin, 2002). Individuals with trauma histories often have poorer health outcomes (Lanius, Vermetten, \& Pain, 2010), although little has been explored in the context of cancer. Trauma history can be a vulnerability factor for increased distress during cancer treatment and can negatively impact HRQOL (Kangas et al., 2002). Furthermore, conceptualizing cancer as a traumatic event in itself can lead to increased distress, decreased HRQOL and poorer coping (Fallah, et al. 2012). 
Despite these challenges, some patients undergoing cancer treatment report positive subjective well-being throughout the experience. How is it possible, when faced with a potentially life-threatening illness, that some patients respond with grace and compassion instead of reflexive distress? One reason may be that they possess psychological resilience, the capacity to overcome psychological distress and adjust positively to the aftermath of potentially traumatic events (Thompson, Arnkoff, \& Glass, 2011). Resilience may be related to mindfulness - present-moment, non-judgmental awareness - a construct that is receiving increasing empirical support as an effective buffer against stressful life events (Grossman, Niemann, Schmidt, \& Walach, 2004).

\section{The Relationship Between Trauma and Cancer HRQOL}

Stressful life events are thought to influence biological processes and behavioral patterns that influence disease risk by causing negative affective states (i.e. feelings of distress, anxiety or depression) that lead to dysregulated biological functions (Cohen, Janicki-Deverts, \& Miller, 2007) and behavior. For example, childhood trauma may cause dysregulation in the hypothalamic-pituitary-adrenal (HPA) axis later in life (Heim, Newport, Mletzko, Miller, \& Nemeroff, 2008). The biological connection between stress and health has been well-documented in cancer (for a complete review, see: Antoni et al., 2006; Cohen et al., 2007; and Lillberg et al., 2003). Behavioral changes that occur in response to stress can include poorer health behaviors (i.e. smoking, drinking, decreased exercise) and poorer adherence to medical regimen (Cohen et al., 2007). Examining the impact of trauma history on the cancer experience is still an emerging domain of study in the empirical literature. Specifically, the relationship between traumatic stress and 
HRQOL has only been recently explored (e.g., Kangas, Williams, \& Smee, 2012; Wachen, Patidar, Mulligan, Naik, \& Moye, 2014).

The impact of trauma history on cancer. In a review that examines the association between trauma history and breast cancer progression, researchers found that women who reported having at least one traumatic event in their past had a significantly shorter time in remission as compared with women who did not have a trauma history (Palesh et al., 2007). From a biological standpoint, this may be due the long-lasting impact of trauma on the stress response system, such as the HPA axis, reducing resistance to tumor growth (Palesh et al., 2007). This biological mechanism has been explored in detail elsewhere and is beyond the scope of this review (see Heim et al., 2008). The important implication of these findings, however, is that changes in biology and behavior related to stressful life experiences can make one more vulnerable to extreme reactions (including post-traumatic stress disorder (PTSD)) to stressors later in life (Green et al., 2000).

Behaviors that might impact the incidence and trajectory of lung cancer may be related to poor health behavior following a traumatic event. For example, Brown and colleagues (2010) found that individuals who experienced a traumatic event in childhood had were more likely to engage in smoking behavior, have a higher incidence of lung cancer, and earlier mortality from lung cancer when compared with patients that did not have a childhood traumatic event. While the mechanism through which lung cancer incidence and earlier mortality is not completely explained through smoking behavior, the connection between prior trauma and a poorer lung cancer experience is clear. 
The prevalence of PTSD incidence in oncology patients has ranged from $2 \%$ to $32 \%$ (Kangas et al., 2002). However, comparison across studies is complex due to differences in procedure, samples and measures (Thompson, Eccleston, \& Hickish, 2011). The journey to a cancer diagnosis is not smooth or linear, and sometimes takes months. These anticipatory months include on-going tests, uncertainty and distress as one waits for the final diagnosis from a doctor. This period of distress, during and after diagnosis, can encompass many of the symptoms of PTSD including intrusive thoughts, re-experiencing aspects of the illness after recovery, avoidant behaviors, having a sense of foreshortened future and hyperarousal (Green et al., 2000).

As many of these symptoms are natural reactions to the cancer experience, to pathologize these reactions with a formal diagnosis of PTSD is insensitive. Within the cancer literature, many studies used self-report measures to assess for PTSD symptoms in a continuous measurement, instead of to categorically diagnose their participants with PTSD. Following this trend, the symptoms related to cancer-related posttraumatic reactions will now be operationally defined as cancer-related posttraumatic stress symptoms (PTSS) for this review unless the authors specified their participants as meeting full diagnostic criteria of PTSD. It should be noted that regardless of conceptualization, the presence of either having PTSS (without meeting diagnostic criteria for PTSD) or having been diagnosed with PTSD, symptoms were related to decrements in HRQOL (e.g., Gold et al., 2012; Shand, Cowlishaw, Brooker, Burney, \& Ricciardelli, 2014).

In a review article that examined the prevalence of cancer-related PTSS in adult cancer patients, Kangas et al (2002) noted that the majority of studies used a cross- 
sectional design to index cancer-related PTSS between 2 months and 12 years following a cancer diagnosis (Kangas et al., 2002). Researchers also observed a high incidence of both intrusive and avoidance symptoms that were specifically related to the cancer diagnosis. Several methodological limitations were noted (e.g., use of cross-sectional designs, insufficient assessment tools, sampling issues) and researchers asserted a need for improved research design, more varied sampling, and the use of gold-standard interview-based diagnostic measures of PTSD for future studies (Kangas et al., 2002). After a review of the literature, these critiques are still relevant today.

Lung cancer as a traumatic stressor. Classifying a potentially terminal illness as a traumatic stressor has been well documented in the cancer literature (Cordova et al., 2007; Gurevich, Devins, \& Rodin, 2002; Wilson \& Keane, 2004). Cancer’s consideration as a traumatic stressor stems from its potential to overwhelm ordinary adaptive capacities to stress (Gurevich et al., 2002). Each stage of the cancer experience (i.e. the risk, diagnosis, treatment, progression and recurrence) may constitute as profoundly stressful events that can elicit cancer-related PTSS (Rodin, Craven, \& Littlefield, 1991). While it may not be a single event, such as diagnosis, but an accumulation of stressful events that promote posttraumatic reactions, the conceptualization of cancer as traumatic event is becoming widely accepted. Those with prior trauma histories, often regard cancer as the worst trauma experienced as compared to others (Alter et al., 1996).

Cancer is a distinct traumatic event as compared to other traumatic stressors, because (1) the traumatic stressor is still present, and (2) symptoms are not due to a misinterpreted fear of an event reoccurring because the feared outcome (i.e. chemotherapy, radiation or death) may in fact occur (Kangas et al., 2002). Cancer is a 
unique traumatic event given its chronic nature, and the psychosocial consequences and uncertainty of the disease trajectory having a devastating impact on HRQOL. Cancer is not only a trauma distinct from other traumatic life events, but it also increases a patient's vulnerability to developing PTSS (Fallah et al., 2012). The stressor that can trigger cancer-related PTSS can be any stressful experience during the cancer trajectory including the testing, diagnosis and treatment (Gurevich et al., 2002).

Lung cancer, specifically, can elicit strong traumatic reactions given the frequently rapid disease trajectory and later-stage diagnosis. In the first study to examine the classification of lung cancer as a Criterion A stressor as defined by both the DSM-IV criteria and the DSM-5 criteria, Andrykowski and colleagues (2015) found that more individuals met stressor criteria utilizing the DSM-5 stressor criterion ( $57 \%$ of patients) as compared to the DSM-IV stressor criteria (37\%). Furthermore, those that identified lung cancer as a Criterion A stressor had poorer psychological outcomes as compared with those that did not, demonstrating a clear relationship between perception of lung cancer as a traumatic event and subsequent psychological distress (Andrykowski, Steffens, Bush, \& Tucker, 2015). The cancer literature has identified several factors that heighten risk of cancer-related PTSS, including early age at diagnosis, prior trauma history, current comorbid depression and anxiety, and avoidant coping style. These risks are now discussed in detail.

Age. Several studies have demonstrated a negative relationship between age and incidence of cancer-related PTSS (Gold et al., 2012; Gonçalves, Jayson, \& Tarrier, 2011; Green et al., 2000; Koopman et al., 2002; Wachen et al., 2014). For example, in a longitudinal study that examined the incidence of PTSS in a cohort of 121 ovarian cancer 
patients, researchers found that between $36 \%-45 \%$ of the total sample experienced cancer-related PTSS over the course of four assessments (Gonçalves et al., 2011). One of the most significant and consistent predictors of developing cancer-related PTSS during the course of treatment was younger age. Researchers speculated that younger women, in whom the disease may be more aggressive, perceived the diagnosis as more traumatic (Gonçalves et al., 2011).

Prior trauma history. Several studies indicated that a history of being exposed to a traumatic event prior to the cancer diagnosis made a patient more vulnerable to developing cancer-related PTSS (Banou, Hobfoll, \& Trochelman, 2009; DuHamel et al., 2001; Gold et al., 2012; Green et al., 2000; Jahn, Herman, Schuster, Naik, \& Moye, 2012; Lutgendorf et al., 2013; Mystakidou et al., 2012; Wachen et al., 2014). For example, both Green and colleagues (2000) and Banou and colleagues (2009) found that adult victimization predicted cancer-related PTSS, while childhood traumatic events did not. Researchers hypothesized two different reasons why adult victimization was significant over childhood trauma: one theory is that trauma survivors are more likely to have intrusive recollections of their most recent trauma experience (i.e. adult victimization) that may be activated during the cancer experience (Green et al., 2000). Another theory focused on the nature of the trauma, and hypothesized that prior adult interpersonal trauma may compound PTSS as interpersonal traumas may deplete available resources (e.g., social support) to cope with subsequent stressors (i.e. cancer; Banou et al., 2009). While the mechanism between prior trauma history and increased vulnerability to cancerrelated PTSS has not been elucidated, a possible cumulative stress burden is clearly demonstrated in the literature. 
Emotional distress. Depression and anxiety are often comorbid disorders with PTSD, and this relationship was found in several cancer populations. Concurrent high levels of both depression and anxiety predicted higher levels of cancer-related PTSS (Lutgendorf et al., 2000; Okamura, Yamawaki, Akechi, Taniguchi, \& Uchitomi, 2005; Shand, Brooker, et al., 2014; Wachen et al., 2014; Wong \& Fielding, 2007). Emotional distress can lead to avoidant coping, which negatively impacts HRQOL (Lutgendorf et al., 2000). Psychological comorbidity can further compromise a patient's emotional and physical well-being (Kangas et al., 2002), indicating a need to screen not only for cancerrelated PTSS, but also for other psychiatric disorders that may be complicating adjustment to the cancer diagnosis and negatively impacting HRQOL.

Other factors. Additional risk factors include lower education and more advanced disease stage (Jacobsen et al., 1998; Smith et al., 2009), shorter time since diagnosis and assessment of PTSS (Sprangers, Tempelaar, van den Heuvel, de Haes, \& Hanneke, 2002), lower perceived social support and prior psychiatric history (Green et al., 2000), and avoidant coping style (Amir \& Ramati, 2002; Lutgendorf et al., 2000; Shand, Brooker, et al., 2014). These factors may contribute to increased cancer-related PTSS and decreased HRQOL.

Research on the relationship between cancer-related PTSS and HRQOL is presently at an early stage. Many studies have examined the incidence or prevalence of PTSS following a cancer diagnosis, however, the direct relationship between PTSS and HRQOL has not been examined thoroughly via longitudinal-prospective studies. Studies that have examined cancer-related PTSS and HRQOL, have found a strong inverse relationship between the two (e.g. Golden-Kreutz et al., 2005; Kangas et al., 2012; Shand 
et al., 2014; Smith, Zimmerman, Williams, \& Zebrack, 2009). The association between cancer-related PTSS and HRQOL has not yet been explored in lung cancer patients and will be examined in this study.

All studies that examined the impact of cancer-related PTSS on HRQOL reviewed demonstrated that higher symptoms of cancer-related PTSS were significantly associated with lower levels of HRQOL (Amir \& Ramati, 2002; Geffen, Blaustein, Amir, \& Cohen, 2003; Gold et al., 2012; Kangas et al., 2012; Shand, Brooker, et al., 2014; Wachen et al., 2014). The risk factors associated with developing cancer-related PTSS (e.g., younger age and prior trauma history) were also associated with decreased HRQOL (e.g., Lutgendorf et al., 2013; Okamura et al., 2005). For example, in a cross-sectional study of veterans, Gold et al (2012) examined the rates of cancer-related PTSS incidence and its relationship with HRQOL. Of the patients included in this heterogeneous cancer population, $23 \%$ were diagnosed with lung cancer. Nearly half (45\%) of the sample met either full diagnostic criteria for PTSD $(n=78)$ or experienced cancer-related PTSS $(n=$ 58). Patients experiencing cancer-related PTSS had significantly lower Karnofsky Performance status scores, higher ratings of mood disturbance, and lower HRQOL when compared with patients that did not experience any cancer-related PTSS symptoms; patients that met diagnostic criteria for PTSD experienced more deficits in outcome measurements as compared to those that did not meet diagnostic criteria (Gold et al., 2012). This demonstrates that the more cancer-related PTSS symptoms a patient experienced, the lower the patient's HRQOL was as a result. This study was the first to examine the relationship between cancer-related PTSS and HRQOL in a heterogeneous cancer population, and demonstrated how patients meeting full diagnostic criteria for 
PTSD and those that only experienced some cancer-related PTSS were both associated with decreases in HRQOL.

In another cross-sectional study that examined cancer-related PTSS in a cohort of veterans, the majority of the sample $(86 \% ; n=166)$ reported at least some cancer-related PTSS, with a small portion of the sample (10\%) meeting full diagnostic criteria for PTSD (Wachen et al., 2014). Researchers also examined the impact of previous combatexposure and combat-related PTSD on current cancer-related PTSS. Results indicated that a third (37\%) of individuals with combat-related PTSD also reported higher cancerrelated PTSS as compared to individuals without combat-related PTSD. Cancer-related PTSS was negatively associated with physical and social HRQOL. The researchers concluded that psychosocial factors, particularly previous trauma histories, need to be considered when treating patients with cancer (Wachen et al., 2014). This study provides a much more comprehensive picture of how previous trauma history, with particular emphasis on current non-cancer related PTSS, can impact HRQOL.

In a recent longitudinal prospective study, researchers examined the impact of prior trauma history on HRQOL in a sample of ovarian cancer patients $(n=123)$ at two time-points: between their initial pre-operative appointment and surgery, and again at a one-year follow-up appointment (Lutgendorf et al., 2013). Researchers found that noncancer related traumatic events that occurred one year prior to surgery, were negatively associated with HRQOL at one-year follow-up post-surgery. This relationship suggests that patients who were exposed to trauma prior to surgery may be less resilient in their recovery from surgery and initial treatment (Lutgendorf et al., 2013). Researchers also found that the number of traumatic events was more strongly associated with decrements 
in HRQOL when adjusting for treatment variables such as having received chemotherapy, suggesting a cumulative stress burden and decreased resources to cope (Lutgendorf et al., 2013). The impact of trauma history on both cancer-related PTSS and HRQOL needs to be explored further as prior trauma history may substantially affect a patient's ability to cope with a cancer diagnosis and treatment. The inverse relationship between cancer-related PTSS and HRQOL has been shown in both cross-sectional and longitudinal studies. A history of trauma appears to complicate both adjustment to a cancer diagnosis and subsequent perception of HRQOL for a cancer patient during treatment.

Limitations of the research. Despite strong evidence of an inverse relationship between trauma and HRQOL, several limitations in research methodology are apparent which may detract from the strength of this association. In a review of the studies published before 2002, Kangas and colleagues (2002) critiqued the literature for being cross-sectional in nature, and this has not changed in the subsequent years. Of the 27 studies reviewed, 19 were cross-sectional. Cross-sectional methodology limits the causal inferences that can be drawn between trauma and HRQOL (Kangas et al., 2002). Longitudinal research that examines the trajectories of cancer-related PTSS and its impact on HRQOL over the course of treatment is needed. For example, researchers found that more patients met diagnostic criteria for cancer-related PTSD during followup assessments than at baseline (Gonçalves et al., 2011). The need for prospective and longitudinal research is necessary when considering that many people who display cancer-related PTSS symptoms in the initial weeks after diagnosis recover in the following three-months without formal intervention (Kangas et al., 2002). This highlights 
how a sample can change over time and multiple assessments are needed to understand the relationship between cancer-related PTSS and HRQOL over the course of treatment. Another limitation noted is the disproportionate demographic representation across studies. For example, there is a clear gender skew with more studies examining women with cancer (the majority with breast cancer) over men. The few studies that did examine the impact of trauma on HRQOL in men were in veteran samples (Hwang, Chang, Fairclough, Cogswell, \& Kasimis, 2003; Gold et al., 2012;Jahn et al., 2012; Wachen et al., 2014). As veterans are a population where the likelihood of having a prior trauma history that may complicate adjustment to cancer is high, more work is needed with this population. Another shortcoming prevalent in the research is that the majority of studies employed predominantly European American participants. While the two studies that examined these relationships in Asian populations found similar results (Okamura et al., 2005; Wong \& Fielding, 2007), these and other minority populations need to be examined further.

Overall, research needs to be extended to represent men, other cancer populations, and minority patients. For example, fears of how a diagnosis will impact their relationships with their partners, and fears of becoming a burden to the family can be barriers to treatment for African American females diagnosed with late-stage breast cancer, and can negatively impact domains of HRQOL differently as compared to European American women (Lannin et al., 1998). These data highlight the importance cultural beliefs when conceptualizing treatment for African American women as compared to European American women. Women are more likely to report mental health problems related to trauma exposure as compared to men (American Psychiatric 
Association, 2013). This is a promising avenue for further research, given different presentations of traumatic stress symptoms in men and minority populations.

A third limitation in research methodology is reliance on self-report measures. The majority of studies utilized self-report measures to infer a diagnosis of PTSD. While self-report measures can give a fairly accurate picture of symptoms, the gold-standard assessment for diagnosing PTSD is in a structured clinical interview such as the Clinician-Administered PTSD Scale (Forbes, Creamer, \& Biddle, 2001). Given the complex and chronic nature of cancer, self-report measures that are better suited to an acute traumatic event may not be accurately capturing the diagnostic presentation of cancer patients. For example, many symptoms that are listed in the diagnostic criteria for PTSD overlap with disease-related factors (e.g. impaired concentration and fatigue; Shand, Brooker, et al., 2014). Given this potential overlap in symptoms, a diagnostic interview may be more appropriate when seeking to give a diagnosis of PTSD.

The limitations noted weaken the strength of the relationship between trauma and HRQOL, and necessitate further study with more rigorous research designs, varied sampling, and more precise measurement instruments to confirm these findings. Little research has been done in the area of lung cancer to determine how the unique experience of lung cancer impacts both cancer-related PTSS and HRQOL. It is worth noting, however, that the findings of all studies examining trauma and HRQOL indicate there is a robust inverse relationship between trauma and HRQOL. Several studies clarified what was causing the posttraumatic reactions (either the cancer or a prior trauma; e.g., Jahn et al., 2012; Mehnert et al., 2014). The careful distinction between prior trauma-related PTSD and current cancer-related PTSS demonstrates a fundamental understanding that 
cancer is potentially a traumatic event itself that may result in posttraumatic reactions. Both prior trauma history and cancer-related PTSS have a negative impact on HRQOL. Understanding the vulnerability factors (e.g., trauma history and cancer-related PTSS) that contribute to poorer HRQOL for cancer patients has both research and clinical implications. The question remains: How do we promote resilience when faced with a cancer diagnosis?

\section{Mindfulness}

The innate resilience seen in some cancer patients and trauma survivors may be the product of mindfulness. Mindfulness first appeared in Western psychology as a behavioral medicine intervention initiated by Jon Kabat-Zinn for patients with chronic pain (Kabat-Zinn, 1990). The construct of mindfulness arose from the dialogue between Western medicine and Buddhist traditions, and evidence for both its popularity and clinical efficacy is growing (Kang \& Whittingham, 2010). Mindfulness-based interventions (MBI) such as Mindfulness-Based Stress Reduction (MBSR; Kabat-Zinn, 1990), Mindfulness-Based Cognitive Therapy (MBCT; Segal, Williams, \& Teasdale, 2002), and Dialectical Behavior Therapy (DBT; Linehan, 1993) are interventions that have been shown to be efficacious in improving both mental and physical outcomes for clinical populations (see Baer, 2003; Brown, Ryan, \& Creswell, 2007; Grossman, Niemann, Schmidt, \& Walach, 2004; Shapiro, Carlson, Astin, \& Freedman, 2006).

Despite a growing popularity, there is a notable lack of consensus in operationally defining mindfulness. The most widely accepted operational definition in Western healthcare applications is "the awareness that emerges through paying attention on purpose, in the present moment and nonjudgmentally to the unfolding experience" 
(Kabat-Zinn, 2003, pg. 145). Mindfulness is thought to exist as an inherent human quality that can be purposefully utilized through meditation and practice (Brown \& Ryan, 2003). Mindfulness has also been characterized as involving self-regulatory capacity (Brown \& Ryan, 2003), as a meta-cognitive skill (Bishop et al., 2004), and as a pathway to acceptance and change (Linehan, Tutek, Heard, \& Armstrong, 1994). This variance in the operational definition complicates the empirical study of the construct.

Grossman (2011) has published several critiques of the mindfulness literature and the state of current assessment tools. The main critique appears to stem from the lack of gold-standard measures with which to assess an individual's level of mindfulness, despite the existence of multiple self-report inventories (Grossman, 2011). Examples of selfreport measures include the Mindful Attention Awareness Scale (MAAS; Brown \& Ryan, 2003), the Toronto Mindfulness Scale (TMS; Lau et al., 2006) and the Kentucky Inventory of Mindfulness Skills (KIMS; Baer, Smith, \& Allen, 2004) to name a few (for a full discussion of mindfulness scales, see Sauer et al., 2013). The various self-report measures focus on separate facets of the overall construct of mindfulness as informed by the researcher's operational definition of the construct. The lack of a gold standard assessment tool brings into question the construct validity of these scales.

Another critique is that current mindfulness measures may assess qualities quite different from the original Buddhist characterization of mindfulness, and do not take into account Buddhist elements of mindfulness such as intention, tolerance, compassion, kindness, and ethical behavior (Grossman, 2008; Grossman, 2011). Grossman (2014) offers a more multifaceted definition of mindfulness as an "act of unbiased, openhearted, equanimous experience of perceptible events and processes as they unfold from the 
moment to moment." Mindfulness appears to be a broad construct, and researchers have attempted to formally operationalize it for research purposes to better understand how it can benefit clinical populations. This process, however, may have led to reductionist measurements that fail to capture the full picture of mindfulness as informed by Buddhist traditions.

Related to the question of how to best define mindfulness, there is also a debate of whether the construct of mindfulness exists as a trait (i.e. dispositional) or a state (i.e. acquired through practice). A trait, or dispositional characteristic, is something that remains stable over time, whereas a state fluctuates over time. In a review of the literature, mindfulness can be characterized as either, or in some cases, both, depending on the research. For example, the ability to be mindful is purported to exist in all individuals, and formal mindfulness practice can increase this ability. This definition implies mindfulness can be both a trait and a state construct (Bishop et al., 2004; Brown, Ryan, \& Creswell, 2007). Both Bishop and Kabat-Zinn assert that the ability to engage in non-judgmental, present moment focus must be present before a mindfulness state can be engaged. Brown and Ryan (2003), suggest that there is a spectrum of mindful capacity along which individual differences lie.

Regardless of whether it is conceptualized as an inherent dispositional trait, or acquired state (or perhaps both), evidence of the salutary effects of mindfulness has begun to accumulate. Based on an analysis of the mindfulness literature, mindfulness will now be conceptualized as the following for the duration of this review: dispositional mindfulness is operationally defined as the innate quality of awareness that exists independent of formal training. In contrast, acquired mindfulness is defined as the change 
in skills seen over the course of an intervention during formal mindfulness practice. Initial work into teasing these constructs apart shows that dispositional mindfulness has been most associated with the mindfulness-based skills of decentering and finding purpose (Pearson, Brown, Bravo, \& Witkiewitz, 2014), while acquired mindfulness has been most associated with the act of reappraisal of negative stimuli (Garland, Hanley, Farb, \& Froeliger, 2013).

While research has shown that MBIs may be effective in improving mental and physical outcomes, the mechanisms of action underlying these interventions are still in question. Several models have been proposed, each suggesting a different underlying mechanism of change. For example, these proposed mechanisms focus on changes in the metacognitive action of reperceiving (Shapiro et al., 2006), decreased rumination (Hawley et al., 2014), increased metacognitive capabilities (Teasdale et al., 2002), and the increased capacity to focus one's attention (Lutz, Brefczynski-Lewis, Johnstone, \& Davidson, 2008).

In a model that is most applicable to trauma survivors and cancer patients, the mindfulness stress-buffering hypothesis proposes that mindfulness acts by mitigating stress appraisals and reduces stress reactivity responses (Creswell \& Lindsay, 2014). A clear example of this hypothesis was previously developed by Salmon and colleagues (2011) and demonstrates the potentially ameliorative effects of mindfulness on stress and health (see Figure 1) 


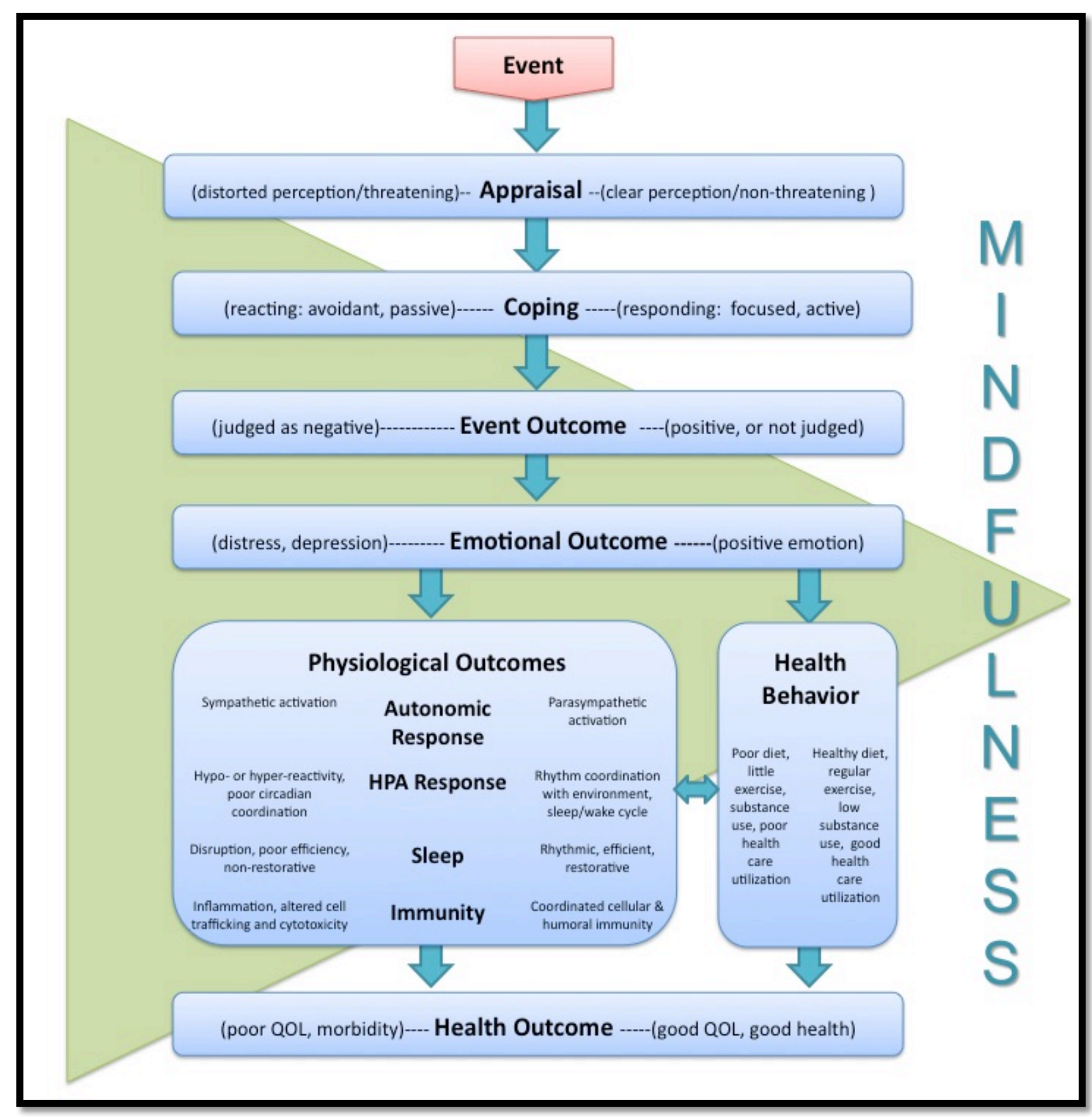

Figure 1: Stress-Reducing Aspects of Mindfulness: Adaptation of the Transactional Model of Stress and Coping (Salmon, Sephton, \& Dreeben, 2010). Reproduced with permission.

Specifically, mindfulness may facilitate the capacity to receptively observe stressors as they arise in the present moment with acceptance. Mindfulness has been shown to mitigate threat appraisals (Brown, Weinstein, \& Creswell, 2012; Weinstein, Brown, \& Ryan, 2009), which can increase the likelihood of using more adaptive coping 
strategies (e.g., improved health behavior or social support seeking; Weinstein et al., 2009).

One intriguing possibility to consider is that mindfulness may shift one's stress appraisals from negative threat-appraisals that engender a powerful biological reaction to an objective perspective, which allows an individual to take a broader viewpoint and choose to respond to the stressor instead of reacting to it. Figure 1 demonstrates how mindfulness may serve to take an individual from passive, avoidant or reactive coping methods to active, focused and responsive management of difficult events (Salmon, Sephton, \& Dreeben, 2011). This shifting perception not only impacts the presentmoment coping with stressful events, but can also affect future health-related outcomes as well. It could be that state, or acquired mindfulness, shifts into trait (i.e. dispositional) over time. At this point, there has been no empirical work into when this possible shift may occur.

The literature shows preliminary support for the mindfulness stress-buffering hypothesis. Initial studies show that mindfulness reduces both psychological and biological stress reactivity to exposure to a variety of laboratory stressors. For example, participants with higher dispositional mindfulness had lower psychological stress reactivity to laboratory stressors (Arch \& Craske, 2010; Weinstein et al., 2009) and acquired mindfulness also reduced blood pressure reactivity to a laboratory stressor in highly stressed community adults (Nyklíček, Mommersteeg, Van Beugen, Ramakers, \& Van Boxtel, 2013). Another study measured the impact of dispositional mindfulness on participants who were completing a high or low stress task, and found that higher dispositional mindfulness predicted lower cortisol responses, lower anxiety and lower 
negative affect in response to the stressor (Brown, Weinstein, \& Creswell, 2012). A notable trend in these studies is that mindfulness stress-buffering effects were most pronounced in the participants that were more stressed (e.g., Arch \& Craske, 2010; Brown et al., 2012). Mindfulness has powerful effects on both psychological and biological pathways of stress reduction (for a complete review of the biological pathways see Creswell, 2014). Therefore, the role of mindfulness in the context of traumatic stress may be a feasible extension of this work.

\section{The Relationship Between Trauma and Mindfulness}

The traumatic stress literature has identified complex ways that trauma impacts various domains of functioning. Despite these advances, there are still limitations in both theory and treatment of posttraumatic reactions, specifically in understanding etiological and maintenance factors (Brewin \& Holmes, 2003). Currently, cognitive-behavioral treatments (CBT), in particular Prolonged Exposure (PE), have the greatest empirical support for trauma treatment, (Watts et al., 2013). However, these techniques appear to be limited in the scope of symptoms they address. Exposure therapy emphasizes the reduction of avoidance (through repeated exposure exercises), and so it may be superior to other treatments in reducing avoidance, but not necessarily better at reducing other features of PTSD, such as the numbing symptoms. Indeed, in a study that examined the efficacy of relaxation treatment, EMDR and PE, none of the three interventions provided more than a 50\% reduction in numbing and hyperarousal symptoms at the end of treatment (Taylor et al., 2003). Among PTSD sufferers who were not responsive to PE, anger and guilt have been identified as the most memorable emotions related to trauma (Grunert et al, 2007). A focus on potential symptoms and emotions that may not respond 
to treatment as readily, as well as their potential maintenance factors, may be an area where future intervention development can be targeted.

Recent developments in the traumatic stress literature reveal a role for MBIs (Baer, 2003; Follette et al., 2006; Orsillo \& Batten, 2005). By increasing both acceptance and skillful behavior, negative effects of traumatic stress may be partially ameliorated (Follette et al., 2006). MBIs have been shown to buffer against the distress caused by PTSD and other disorders (Bernstein, Tanay, \& Vujanovic, 2011; Michal et al., 2007; Smith et al., 2011). The theoretical basis for this finding arises from the potential overlap of mechanisms in mindfulness and posttraumatic reactions, as well as responses that extend beyond symptoms of PTSD. For example, many individuals recovering from trauma engage in avoidant behaviors, which is the antithesis of mindful behavior (Follette, Palm, \& Pearson, 2006). By engaging in mindful behaviors, the cycle of avoidance is broken, and the individual can move towards more adaptive coping. The initial work in the area of mindfulness and traumatic stress provides preliminary evidence for the efficacy of these types of approaches for cancer patients (Becker \& Zayfert, 2001; Thompson \& Waltz, 2010).

Individuals who experience trauma symptoms often exhibit behaviors that reflect a narrowing of habitual responses in reaction to aversive internal experiences and can cause perseverative cognitions and feelings of numbing (Follette, Palm, \& Pearson, 2006). These behaviors can include avoiding certain places, people or situations that may prompt a negative reaction. This narrowing of behavioral responses has been attributed to psychological inflexibility, due to being less likely to utilize mindfulness-based skills (Follette, Palm, \& Hall, 2004), and involves the increased use of avoidant behaviors. This 
possible lack of mindfulness-based coping resources can contribute to avoidance of triggering stimuli and an unwillingness to engage in the present-moment due to increased distress.

Mindfulness is in direct conflict with this type of response, as it is associated with a greater willingness to engage painful thoughts and emotions (Thompson \& Waltz, 2008). This may serve to facilitate improved self-regulation, and increased self-care behavior (Allen \& Leary, 2010). An example of this is the wiliness to practice positive cognitive restructuring and treating oneself with kindness and compassion (Allen \& Leary, 2010). Individuals recovering from trauma exposure often struggle with selfcritical thoughts and remaining in the present moment, as evidenced by a tendency to ruminate on the past or to worry about the future. Such cognitive 'time travel' into the past and future markedly detracts from their capacity to direct and sustain attention in the present. Mindfulness helps to anchor the individual in present moment experience and offers an opportunity to explore alternative options, in terms of cognitive and behavioral responses by giving the individual space to respond to distressing events with kindness instead of reflexive distress.

Applying and empirically studying the efficacy of MBIs for individuals recovering from traumatic stress is a recent development in the last two decades. Despite its nascency in the clinical research literature, it has become apparent that there is a place for MBIs for those recovering from trauma. The studies published within the last two decades show promising results linking mindfulness and trauma outcomes. All of the studies that examined the relationship between trauma and mindfulness found a strong inverse relationship. Specifically, cross-sectional studies found that higher levels of 
dispositional mindfulness were associated with lower levels of PTSD or trauma-related psychopathology (Bernstein et al., 2011; Michal et al., 2007; Thompson \& Waltz, 2010; Smith et al., 2011; Vujanovic, Youngwirth, Johnson, \& Zvolensky, 2009). What is interesting about these studies is that despite agreeing on the overall level of mindfulness being inversely related to decreased levels of PTSD, the mindfulness skill that accounted for the variance was different across studies.

For example, two studies that reported that the 'acceptance without judgment' subscale, as measured by the KIMS, was most strongly associated with decreased PTSDrelated distress (both measured by the Posttraumatic Diagnostic Scale; Thompson \& Waltz, 2010; Vujanovic et al., 2009). Vujanovic and colleagues (2009) investigated the incremental validity of mindfulness-based processes in relation to PTSD-symptom severity in 239 healthy trauma-exposed adults and found that the acceptance without judgment subscale was significantly incrementally associated with all PTSD symptom clusters. Thompson and Waltz (2010) examined the relationship between mindfulness and experiential avoidance, a proposed maintenance factor of PTSD. They found that nonjudgment accounted for a unique portion of the PTSD avoidance symptom cluster in a cohort of 378 healthy trauma-exposed adults with PTSD. These findings indicate that a subset of mindfulness skills, such as pre-existing levels of nonjudgmental acceptance, may counteract avoidant coping strategies often employed by individuals coping with trauma.

Mindful attention and awareness are another set of mindfulness skills that were also seen to be negatively associated with PTSD symptoms ( Bernstein et al., 2011; Vujanovic et al., 2009). Mindful attention refers to the ability to self-regulate your 
attention to the immediate experience and mindful awareness refers to the conscious introspective attentiveness to your experience (Van Dam, Earleywine, \& Borders, 2010). Vujanovic and colleagues (2009) found that the 'acting with awareness' subscale of the KIMS was associated with decreased levels of re-experiencing symptoms in healthy trauma-exposed adults. Bernstein and colleagues (2011) found that in a sample of 76 healthy trauma-exposed adults, higher levels of mindful attention and awareness, as measured by the MAAS, were negatively associated with PTSD symptom severity, psychiatric multi-morbidity, anxious arousal and depression. These findings broadly support the hypothesis that mindful attention and awareness skills may be negatively associated with PTSS severity by counteracting habitual avoidance related to the trauma.

While these cross-sectional studies do address the relationship between trauma and mindfulness, causal inferences and relationships cannot be delineated due to the study designs; longitudinal studies can begin to explore causal relationships in more detail. Longitudinal studies that have begun to investigate the relationship between acquired mindfulness and trauma report positive outcomes. It appears that MBIs are both feasible and efficacious in increasing mindfulness skills in trauma-exposed adults and decreasing PTSD symptoms (e.g., Bormann, Oman, Walter, \& Johnson, 2014; Kearney et al., 2013; King et al., 2013). These studies have begun to examine not only the effects of MBIs on trauma-exposed adults, but also the mechanisms of action as well.

Researchers noted a connection between engagement in mindfulness skills use, compliance with the intervention, and decreased PTSS post-intervention suggesting a possible dose-response effect for mindfulness skills use over time. For example, in a study that investigated the impact of MBSR on 47 military veterans, those that attended 
four or more MBSR sessions had greater clinically significant improvements in PTSD and depression symptoms than the control group (Kearney, McDermott, Malte, Martinez, \& Simpson, 2013). These results suggest that greater engagement in a MBI yields more positive results indicating a possible dose-response effect.

Using more sophisticated methodology, two studies examined the impact of a MBI using a randomized controlled trial (RCT) design. King and colleagues (2013) examined the impact of MBCT on a group of 37 veterans that were randomly assigned to either the MBCT group or the treatment as usual group. Results showed that the MBCT group had significantly more improvements in PTSS as compared to the control group (King et al., 2013). Bormann and colleagues (2014) assigned 146 veterans diagnosed with PTSD to either a MBI that included mantra repetition or a treatment as usual group. The intervention group had significantly more improvements in both levels of mindfulness and PTSS reduction as compared to the control group (Bormann et al., 2014a). These recent studies demonstrate that MBIs for veterans with PTSD are both feasible and more efficacious in reducing posttraumatic reactions, compared to treatment as usual (i.e. psychotherapy).

Recently published studies have also begun to address the question of how mindfulness may work in reducing PTSS. Three studies found that increased levels of acceptance (the willingness to engage in experiences without the intention to change) were associated with decreases in PTSS (Goldsmith et al., 2014; Kearney, McDermott, Malte, Martinez, \& Simpson, 2012; Kearney et al., 2014). Kearney and colleagues (2012) piloted an MBSR intervention for 90 veterans diagnosed with PTSD and found that increased levels of acceptance were associated with improvements in PTSD (avoidance, 
emotional numbing and hyperarousal) at the six-month follow-up. Goldsmith and colleagues (2014) piloted an MBSR intervention for ten trauma-exposed adults and found that levels of PTSD, depression and shame-based trauma appraisals decreased while levels of acceptance increased post-intervention. Kearney and colleagues (2014) examined the impact of a meditation-based intervention on 42 veterans with PTSD and reported increased pleasant emotions, decreased unpleasant emotions, and increased acceptance immediately following the intervention and at three-month follow-up. Increased levels of acceptance appear to help individuals engage in more adaptive coping strategies, instead of avoiding stimuli related to the trauma experience.

Two recent studies examined the potential mediating effects of mindfulness on trauma symptoms (Bormann et al., 2014; Kearney, McDermott, et al., 2013). Kearney and colleagues (2013) examined the impact of a MBI for 42 veterans diagnosed with PTSD and found that increased levels of self-compassion, a component of mindfulness, mediated improvements seen in both PTSS and depression. Theory suggests that mindfulness is a necessary prerequisite to self-compassion, as mindfulness allows one to clearly see internal experiences as they arise (Neff, 2003b). In fact, research has demonstrated that changes in mindfulness predict changes in self-compassion (Birnie, Speca, \& Carlson, 2010) and that cultivating self-compassion explains much of the success from MBIs (Kuyken et al., 2010). Bormann and colleagues (2014) also found that increased levels of mindful attention mediated reduced PTSD and depression symptoms, and improved psychological well-being. Both studies found evidence that increased levels of mindfulness mediate reductions in PTSD. 
The mindfulness stress-buffering hypothesis may explain how mindfulness can protect against traumatic stress. In this context, mindfulness may offer a buffering effect against developing PTSD or improving gains when an individual participates in an MBI (Creswell \& Lindsay, 2014). Initial cross-sectional studies indicate support for this possible buffering effect of dispositional mindfulness on PTSD symptoms (Bernstein et al., 2011; Michal et al., 2007; Smith et al., 2011; Thompson \& Waltz, 2010; Vujanovic et al., 2009). Additionally, individuals suffering from traumatic stress symptoms also experience exceptional benefits from participating in an MBI with significant gains in outcome measures. The mindfulness stress-buffering hypothesis has yet to be studied in individuals recovering from traumatic stress, but preliminary research appears to lend support.

Limitations of the research. Despite having both cross-sectional and longitudinal studies that examine the relationship between trauma and mindfulness, there are several methodological limitations in this area. The most significant limitation is a distinct lack of cultural and ethnic variability. The vast majority of studies published in the last decade were in predominantly male, European American veteran populations (Bernstein et al., 2011; Boden et al., 2012; Jahn et al., 2012; Kearney et al., 2012; Kearney, Malte, et al., 2013; Kearney, McDermott, et al., 2013; Vujanovic et al., 2009;). Given that veterans face unique stressors in combat, the extent to which results may be generalized to other trauma populations is limited. Future studies need to examine diverse populations to explore whether effects generalize across gender and trauma type.

Another limitation noted is that the majority of studies reviewed were pilot and feasibility studies with small sample sizes, a fact noted by several study authors (Boden 
et al., 2012; Goldsmith et al., 2014; Jahn et al., 2012; Kimbrough, Magyari, Langenberg, Chesney, \& Berman, 2010; King et al., 2013). A small sample can limit statistical power, reducing, or enlarging, possible effect sizes and detracting from external validity. This can lead to overestimates of effects and low reproducibility of results (Button et al., 2013). Despite this potential publication bias, mindfulness appears to have an effect that is large enough in magnitude to produce significant results despite small sample sizes. This may reflect the "file-drawer" issue where only studies that produce significant results are being published.

The most common limitation noted in this subset of studies is a reliance on selfreport measures of both mindfulness and PTSD symptoms (Boden et al., 2012; Carlson, Silva, Langley, \& Johnson, 2013; Jahn et al., 2012; Kearney et al., 2012; Kearney, McDermott, et al., 2013; Michal et al., 2007; Smith et al., 2011; Thompson \& Waltz, 2010; Vujanovic et al., 2009;). A common criticism of mindfulness research is the lack of gold-standard measurement tools (Paul Grossman, 2011). This lack of clarity in the mindfulness literature resulted in several different measures being utilized across studies. As mentioned previously, diagnostic interviews are the most accurate way to assess for PTSD and very few studies employed this assessment method.

A final limitation to note is that the two RCTs did not utilize an active treatment comparison group, but instead used treatment as usual groups ( Bormann et al., 2014; Kearney, McDermott, et al., 2013). The statistically significant differences between two different active interventions offers stronger support to the power of the intervention as opposed to comparing it to standard care. It also removes the potential confounding 
variable of increased attention inherently given to those in the intervention group compared to a standard care group.

In sum, there is initial support for the positive impact of mindfulness on trauma symptoms and recovery. Although the mechanisms are unclear, increased mindfulness appears to decrease PTSS and improve well-being. Mindfulness may be particularly suited for patients recovering from trauma-exposure as mindfulness interventions help the participant to be increasingly focused in the present moment. This results in increased psychological flexibility when targeting avoidance by increasing attention and purposeful behavior that is often lacking in individuals with trauma histories (Follette et al., 2006). A facet of mindfulness that is encouraged throughout interventions, but has yet to be explored in the trauma literature, is the idea of decentering from one's emotions. The capacity to decenter is the ability to perceive thoughts and feelings as both impermanent and objective occurrences in the mind (Hofmann, Glombiewski, Asnaani, \& Sawyer, 2011) and is particularly salient for those recovering from traumatic stress as elements of self-blame and guilt are often common.

\section{The Relationship Between Cancer HRQOL and Mindfulness}

The capacity to decenter also has powerful implications for the lung cancer experience. As previously discussed, the diagnosis of lung cancer can be traumatic and can compromise HRQOL. Shame and guilt are also commonly present in patients with lung cancer and can exacerbate traumatic stress and promote the use of avoidant coping. Learning to decenter from one's reactions allows for more adaptive coping, increased self-compassion and the ability to choose how to respond. The lung cancer experience is often associated with physical and psychosocial impairments with pain, fatigue, 
depression and anxiety being the most common complaints for patients undergoing treatment. Acceptance, a key component of mindfulness, fosters an ability to embrace change and to acknowledge the reality of the situation by allowing an individual to decenter from thoughts of blame, and to face the present moment challenges with objectivity (Carlson et al., 2010: Ott, Norris, \& Bauer-Wu, 2006). This allows the patient to more readily cope with their diagnosis by actively engaging in their treatment and present-moment experience.

Given the unique psychosocial challenges posed by a lung cancer diagnosis, mindfulness is a well-suited intervention. Interventions, such as MBIs, designed to enhance coping with stress and to promote relaxation are appropriate for lung cancer patients given the chronicity of cancer and the complex emotional and physical stressors often associated with diagnosis and treatment (Ott et al., 2006). As mindfulness has proven to be an efficacious psychosocial intervention for cancer patients, it is no surprise that the study of mindfulness has become a focal point in cancer research in the last two decades (Ott et al., 2006). This focus on examining the impact of mindfulness on cancer outcomes, however, has yet to reach lung cancer patients (Shennan, Payne, \& Fenlon, 2011). The efficacy of MBIs such as MBCT, MBSR and Mindfulness-Based Cancer Recovery (MBCR; Carlson et al., 2010) have shown that some cancer patients respond well to MBIs. MBIs seem to be especially effective for symptoms of stress, depression, anxiety and reduced HRQOL (Cramer, Lauche, Paul, \& Dobos, 2012).

Both cross-sectional and longitudinal studies that have examined the impact of mindfulness on cancer outcomes report decreased distress and increased HRQOL (e.g., Stafford et al., 2013; Tamagawa et al., 2013; Witek-Janusek et al., 2008). These positive 
effects have been noted across a range of cancer populations. The effectiveness of MBIs in cancer treatment has been extensively reviewed elsewhere (see Ott et al., 2006;

Carlson et al., 2010; Cramer, Lauche, Paul, \& Dobos, 2012; Piet, Würtzen, \& Zachariae, 2012). Substantial evidence supports the efficacy of mindfulness as an intervention for cancer, and a brief summary of relevant research is presented below.

Despite the abundance of research in this area, dispositional mindfulness in the context of cancer has not been studied in detail. In only two cross-sectional studies that examined the impact of dispositional mindfulness on cancer outcomes, researchers found that higher levels of dispositional mindfulness were associated with decreased distress and mood disturbance (Tamagawa et al., 2013) as well as improved sleep and psychological outcomes (Garland, Campbell, Samuels, \& Carlson, 2013). Specifically, higher levels of dispositional mindfulness skills such as acting with awareness, nonjudging and non-reacting were associated with improved sleep, psychological status and improved mood (Garland, Campbell, et al., 2013). These results echo previous findings in other populations (e.g., trauma) that assert that increased use of dispositional mindfulness skills prior to an intervention is related to increased positive outcomes and greater natural resilience to stressful life events.

The majority of mindfulness research in cancer has involved longitudinal studies examining the efficacy of MBIs for diverse forms of cancer. These studies have consistently found positive outcomes post-intervention such as decreased depressive symptoms (Altschuler, Rosenbaum, Gordon, Canales, \& Avins, 2012; Ando et al., 2009; Würtzen et al., 2013) and anxiety (Bränström, Kvillemo, Brandberg, \& Moskowitz, 2010; Carlson \& Garland, 2005; Chambers, Foley, Galt, Ferguson, \& Clutton, 2012; Stafford et 
al., 2013; Würtzen et al., 2013). For example, in an RCT that examined the impact of MBSR on depression and anxiety for 336 women with breast cancer, women randomized to the MBSR group had significant improvement in both depression and anxiety as compared to the wait-list control group (Würtzen et al., 2013). These effects were maintained at both the six-month and 12-month follow-up assessments (Würtzen et al., 2013). Mindfulness has been shown to not only effectively improve negative emotional outcomes quickly, but to also be an effective coping mechanism long-term.

Not only has mindfulness been shown to reduce symptoms of psychopathology, but it has also been shown to improve mood as well (Altschuler et al., 2012; Bränström et al., 2010; Carlson \& Garland, 2005; Hoffman et al., 2012). In an RCT that assessed the effectiveness of MBSR on mood, HRQOL and well-being for 229 women with breast cancer, women who were randomly assigned to the MBSR group had statistically significant improvements in mood disturbance (as measured by the Profile of Mood States), and HRQOL both at eight and 12-week follow-up as compared to the control group (Hoffman et al., 2012). Similar to the other RCT noted above, researchers found evidence for long-term effects of mindfulness on well-being, HRQOL and decreased mood disturbance. These results further elucidate both the immediate and long-term gains of MBIs for cancer patients.

As emotional disturbances and other negative outcomes have decreased as a result of MBIs, subjective well-being and increased HRQOL have also been reported across studies (Altschuler et al., 2012; Carlson, Speca, Patel, \& Goodey, 2003; Hoffman et al., 2012; Stafford et al., 2013; Witek-Janusek et al., 2008). One study that piloted an MBSR intervention for 96 women recently diagnosed with breast cancer found that women who 
self-selected to join the MBSR group showed improved immune function, cortisol levels, HRQOL and increased coping effectiveness as compared to the non-MBSR group (Witek-Janusek et al., 2008). Specifically, researchers found that the psychologicalspiritual and family domains of HRQOL were most affected by the MBI. The selfselected method of sampling may have biased the results and should be interpreted with caution. Regardless, the researchers posit that mindfulness may facilitate individuals finding more meaning in family and psychological-spiritual domains and that mindfulness may not directly impact the health and socioeconomic components of HRQOL (Witek-Janusek et al., 2008). Learning to utilize mindfulness skills during the period of adjustment to a cancer diagnosis may lead to more effective coping and improved HRQOL.

For those that specifically measured the construct of mindfulness, researchers found that MBIs led to increased levels of mindfulness post-intervention (Bränström et al., 2010; Chambers et al., 2012; Stafford et al., 2013). In one study that examined the efficacy of group-based MBCT for 50 women with breast or gynecologic cancer, researchers found that there were improvements in distress, HRQOL, mindfulness and posttraumatic growth post-intervention (Stafford et al., 2013). Researchers noted that scores of distress, HRQOL and posttraumatic growth improved as a function of increased mindfulness at each time point and that these gains were evident at three-month followup. This indicates that mindfulness has a direct effect on outcome measurements, such as HRQOL.

In another study, 71 adults with heterogeneous cancer diagnoses were randomized into an MBSR group or a waitlist control group and data was collected over three time 
periods over the course of 6 months (Bränström et al., 2010). Compared to the control group, the mindfulness group had decreased stress, fewer posttraumatic avoidance symptoms, improved positive states of mind, and increased mindfulness. Increased mindfulness scores mediated the decreases in perceived stress, posttraumatic avoidance and improvements in positive states of mind (Bränström et al., 2010). These results indicated that increased levels of mindfulness explained how some of these improvements were facilitated throughout the intervention. As seen in other samples (e.g., trauma) increased mindfulness can potentially mediate positive outcomes for cancer patients and improve HRQOL.

The mindfulness stress buffering hypothesis posits that mindfulness may offer a protective effect against the pathogenic processes of tumor growth and metastasis that are affected by stress (for a complete review of proposed biological processes, see Creswell, 2014). From a psychosocial standpoint, the mindfulness stress-buffering hypothesis proposes a pathway that mindfulness may act on highly stressed individuals in order to bring them to a place of acceptance and peace. The pervasive sense of uncertainty in the period before and after a cancer diagnosis causes considerable stress and sometimestraumatic reactions. There are three phases that many cancer patients work through when facing a cancer diagnosis: denial and disbelief, turmoil, and adjustment (Holland \& Lewis, 2000). Mindfulness may help a patient navigate these phases more readily. The initial cross-sectional work in cancer patients supports this hypothesis as higher levels of dispositional mindfulness were associated with improvements in outcomes (Garland et al., 2013; Tamagawa et al., 2013). 
Additionally, the mindfulness stress buffering hypothesis asserts that individuals that are highly stressed with health conditions will benefit most from MBIs (Creswell, 2014). Longitudinal studies that have examined the impact of mindfulness on stress and related outcomes have found that mindfulness has been both efficacious and long-lasting in promoting improved HRQOL (e.g., Bränström et al., 2010; Stafford et al., 2013). Initial studies in the cancer and mindfulness literature appear to support this model of coping. Additional work is needed to examine whether these initial results are supported within a lung cancer population.

Limitations of the research. Despite evidence that demonstrates the positive impact of MBIs on HRQOL, there are several limitations worth noting. First, only two studies have examined the impact of dispositional mindfulness on cancer outcomes (Garland, Campbell, et al., 2013; Tamagawa et al., 2013). While cross-sectional studies are limited in that they are unable to infer causality, they do address the question of how dispositional mindfulness may be associated with the cancer experience at initial diagnosis and might offer hypotheses regarding natural resilience in the face of cancer. Research into the potential protective effect of dispositional mindfulness on cancer reactions is something that needs to be explored further in future work.

Another limitation is that the majority of studies are pilot and feasibility studies (Altschuler et al., 2012; Ando et al., 2009; Carlson et al., 2003; Carlson \& Garland, 2005; Chambers et al., 2012; Stafford et al., 2013; Witek-Janusek et al., 2008). For the few RCTs that were reviewed, none included an active control arm (Hoffman et al., 2012; Würtzen et al., 2013). As noted above, the conclusions drawn from pilot studies and RCTs that do not include an active control arm are limited. 
The most significant limitation of the literature, however, is the lack of diversity in sampling. All but one of the studies examined for this review were published with a sample consisting of predominately European American female breast cancer patients. This limitation has been noted in previous reviews of the cancer and mindfulness literature (e.g., Ott et al., 2006) and remains a relevant critique. The one study that examined the impact of an MBI for 19 men with prostate cancer found significant improvements in all outcome variables (Chambers et al., 2012). This study indicates the potential efficacy of MBIs for men with cancer and needs to be explored further. Having more diverse samples that include other cancer types, such as lung cancer, and that are more representative on all demographic variables (e.g., gender, age, ethnicity and SES) will allow for greater generalizability to the diverse populations of individuals that are diagnosed with cancer. Only two studies included a sampling of lung cancer patients (Altschuler et al., 2012; Garland, Campbell, et al., 2013); however neither study provided data that was specific to the lung cancer participants.

Despite these limitations, there is ample support for the positive impact of mindfulness on HRQOL. Although the mechanisms have not been explicitly explored, increased levels of mindfulness appear to decrease distress and improve HRQOL. Mindfulness training, with its focus on being present, fostering acceptance and being kind to oneself, may be an antidote to worrying about the future, and self-blame commonly experienced in patients with lung cancer (Glinder \& Compas, 1999). The development of acceptance and decentering through mindfulness practice may directly target the reactive processing that can both cause and worsen psychological distress and diminish HRQOL 
(Stafford et al., 2013). Exploring how mindfulness may serve to foster resilience in the face of lung cancer is both challenging and needed at this stage.

\section{Integration}

After reviewing the literature that examines the relationships between trauma, cancer HRQOL and mindfulness, it is apparent that while these areas of have been examined separately in other cancer types, very little work has begun to investigate these relationships within a lung cancer population. It is also apparent that these areas have been empirically researched in fundamentally different ways. For example, while the relationship between trauma and cancer HRQOL has been largely studied using crosssectional methodology, the relationship between trauma and mindfulness has been evaluated using both cross-sectional and longitudinal designs. Moreover, the relationship between cancer HRQOL and mindfulness has been studied in largely prospective longitudinal methodologies. Integrating the areas of trauma, cancer HRQOL and mindfulness into a cohesive narrative poses two different research questions that are related to the controversy of mindfulness mentioned previously: is mindfulness a trait (dispositional mindfulness)? Or, is it a changing state (acquired mindfulness)?

Mindfulness is best described as a dynamic process (Creswell, 2014). How a researcher conceptualizes mindfulness directly informs the study of the construct itself. For example, mindfulness in the context of trauma has been viewed as both a dispositional trait that can buffer the effects of trauma (e.g., Bernstein et al., 2011) and as an acquired skill that can aid in recovery from a traumatic event (e.g., Kearney, McDermott, et al., 2013). The study of mindfulness in the context of cancer HRQOL, however, has generally conceptualized mindfulness as an acquired skill that informs 
recovery and improves well-being (e.g., Hoffman et al., 2012). These contrasting ways of conceptualizing mindfulness suggest two different models through which mindfulness may operate to promote well-being.

In this regard, mindfulness may serve as both a moderator and a mediator in response to traumatic stress in lung cancer patients. A moderator is a variable that effects the direction and strength of the relationships between an independent predictor variable and a dependent variable (Baron \& Kenny, 1986). This indicates that dispositional mindfulness may act as a moderator in the association between trauma and HRQOL by altering the strength of this association. Mediators explain how or why (i.e. the mechanism through which) the independent variable is able to influence the dependent variable (Baron \& Kenny, 1986). This indicates that acquired mindfulness may be useful to understand the process through which trauma influences change in HRQOL (i.e. acquired mindfulness as a mediator mechanism of trauma effects). Hence, it can be conceptualized that changes in mindfulness can cause changes on trauma levels, which in turn helps to account for changes in HRQOL.

\section{Dispositional mindfulness as a moderator of the effects of trauma on}

HRQOL. In the context of trauma and lung cancer HRQOL, dispositional mindfulness may provide an explanation for why some people are able to face the traumatic experience of a cancer diagnosis with grace. A moderation model may explain how dispositional mindfulness might alter the inverse relationship between trauma and cancer HRQOL. As previously discussed, dispositional mindfulness is an innate human quality, and the ability to engage in non-judgmental, present moment focus is something that all people are capable of (Brown \& Ryan, 2003). Individuals that have higher levels of 
dispositional mindfulness may adjust to the potentially traumatic experience of lung cancer more easily. This innate capacity to be mindful may potentially explain the resilience seen in many traumatized populations.

One cross-sectional study that examined the impact of dispositional mindfulness on psychological health among women with breast cancer noted that high levels of dispositional mindfulness were related to fewer distress-related symptoms and less mood disturbance (Tamagawa et al., 2013). Unfortunately, these researchers did not test for moderation. Given that the outcome variables varied as a function of mindfulness levels, it has been strongly recommended that future work test for moderation (Tamagawa et al., 2013). The literature has shown that patients with high levels of dispositional mindfulness were less likely to experience psychopathological symptoms and engage in maladaptive coping behaviors resulting in more positive outcomes, such as increased HRQOL (Bernstein et al., 2011; Smith et al., 2011; Tamagawa et al., 2013). These findings together suggest that dispositional mindfulness may be an important protective factor for cancer patients.

In an RCT that examined the impact of a brief MBI on 66 healthy volunteers, researchers examined the interactive effects of dispositional mindfulness, and mindfulness meditation training on stress reactivity. Researchers found the following: (1) participants in the mindfulness training group had significantly lower stress perceptions compared to the control group; (2) participants with higher levels of dispositional mindfulness had lower stress perceptions overall; (3) and these main effects were qualified by their interaction. Specifically, receiving mindfulness training or reporting higher dispositional mindfulness buffered self-reported psychological stress (Creswell, 
Pacilio, Lindsay, \& Brown, 2014). These results demonstrate that not only does dispositional mindfulness buffer the effects of stress, but that mindfulness training (or increasing acquired mindfulness) is also efficacious in relieving the stress burden as well. Hence, there is evidence that the conceptualization of mindfulness as a dynamic construct that exists as both a dispositional trait and an acquired state is correct. These results also suggest that mindfulness can be trained to foster emotion regulation and active coping to relieve stress. However, this study is limited in its applicability to lung cancer patients because it was in healthy individuals and did not consider the impact of trauma.

\section{Acquired mindfulness as a mediator of the effects of trauma on HRQOL.}

Acquired mindfulness may operate as a mediator to promote well-being for traumatized lung cancer patients. The first three relationships in this potential model have been discussed in the earlier pages of this review; the novel piece to this model is the suggestion of mediation between trauma and lung cancer HRQOL through acquired mindfulness. The model first suggests that there will be a negative and inverse relationship between trauma and HRQOL in lung cancer patients. This may happen in two ways: (1) trauma history exacerbating adjustment to the cancer diagnosis (e.g., Jahn et al., 2012) and (2) having the cancer diagnosis itself being conceptualized as a traumatic event (e.g., Gold et al., 2012). The second relationship illustrated in the model asserts the inverse relationship between trauma and mindfulness. As shown in the literature, as levels of mindfulness increase, symptoms of PTSD decrease (e.g., Kearney et al., 2012) which can increase levels of HRQOL. The third relationship portrayed in the model is the positive association between cancer HRQOL and mindfulness. This relationship is 
supported by research demonstrating a positive association between HRQOL and participation in MBIs (e.g., Carlson et al., 2003) through longitudinal study designs.

In one study that has tested possible mediation of acquired mindfulness on psychological outcomes in cancer patients, including measures of trauma and HRQOL, researchers found that changes in outcomes were mediated by the changes in mindfulness (Bränström et al., 2010). This study shows the impact of acquired mindfulness on improvements in both cancer-related trauma symptoms and HRQOL (Bränström et al., 2010). The researchers, however, did not examine the potential mediating impact of mindfulness on trauma symptoms and HRQOL together, so the question of how these constructs interact is still lingering.

Acquired mindfulness may mediate the relationship between trauma and HRQOL in lung cancer patients by mitigating the stress appraisals and reducing stress-reactivity responses (Creswell \& Lindsay, 2014). For example, trauma increases one's tendency to become hypervigilant and avoidant of present-moment experiences, hence decreasing one's coping resources and enabling potential misappraisal of ambiguous stimuli as threatening (Follette et al., 2004). This shift in perception may enable more adaptive coping and foster improved HRQOL. The study of mindfulness as a mediator of trauma and HRQOL empirically proposes this potential interaction.

Research shows that participants with trauma histories and current cancer-related PTSS respond well to MBIs and improve in measurements of well-being postintervention in longitudinal studies. Specifically, engagement in the MBI and increased mindfulness skills use has been associated with decreased PTSS (Carlson et al., 2013; 
Goldsmith et al., 2014; Kearney, McDermott, et al., 2013; Kearney et al., 2014) and improved cancer HRQOL (Bränström et al., 2010; Stafford et al., 2013). MBIs teach individuals to be more tolerant and open to present-moment experience. This fosters more proactive coping instead of avoidant coping, and can lead to improved outcomes. These relationships suggest that increased levels of mindfulness skills use, such as acceptance, may be a mechanism through which mindfulness improves HRQOL in the context of traumatic stress.

It would appear that mindfulness has strong implications for lung cancer patients. The current proposed conceptualization of mindfulness as both a dispositional trait and acquired state would provide structure to explore our understanding of the evolving nature of mindfulness research. Trauma history engenders an increased risk for maladaptive adjustment to a lung cancer diagnosis. The trauma of the lung cancer diagnosis itself can exacerbate pre-existing PTSS making the patient more reactive, which can negatively impact HRQOL. Mindfulness, however, may buffer the stress reactivity by fostering more engaged and active coping to enhance HRQOL during the stressful time prior to, and following, a lung cancer diagnosis. Examining the impact of both of these constructs (trauma and mindfulness) together on HRQOL is novel and relevant to lung cancer patients. According to the trauma literature, dispositional mindfulness serves as a protective factor that may foster innate resilience to later stressful circumstances. Research suggests additional training only enhances the use of these latent skills and can improve outcomes. The review of the literature conducted here suggests that cancer patients with high levels of dispositional mindfulness may be less vulnerable to negative psychological outcomes, and that cancer patients can increase acquired 
mindfulness through MBIs to increase HRQOL. In this regard, both dispositional and acquired mindfulness may operate within the same cancer population to promote wellbeing.

The question of how mindfulness may moderate and/or mediate the relationship between trauma and HRQOL in lung cancer patients is not necessarily straightforward. Findings strongly suggest that the addition of mindfulness skills to psychosocial treatment for trauma may enhance both treatment efficacy and acceptability (Follette et al., 2004). Increased use of mindfulness skills has resulted in significant improvements in PTSS, depression, mindfulness and HRQOL for cancer patients (Hoffman et al., 2012; Kearney, McDermott, et al., 2013). These findings suggest that increasing the level of acquired mindfulness, and utilization of mindfulness-based skills (e.g., decentering), may lead to better outcomes.

Individuals who are recovering from a traumatic event, such as lung cancer, often present with attention deficits and functional impairments that are characterized by limited awareness and emotional numbing (Vasterling et al., 2002). Mindfulness can be conceptualized as an emotion regulation strategy by transforming negative emotions into a more positive feeling state by addressing them with kindness, non-judgment and equanimity (Creswell \& Lindsay, 2014). Mindfulness also engenders self-compassion, which seeks to increase feelings of self-assurance and self-soothing by giving individuals the tools to confront negative stimuli with kindness and forgiveness (Neff, 2003). These shifts in a patient's worldview may help to foster improved HRQOL, and improve both physical and mental health outcomes for lung cancer patients. 


\section{Focus of Current Study}

Examining the potential protective role of mindfulness for lung cancer patients within the context of trauma has both research and clinical implications. The empirical literature demonstrates that trauma history and the perception of cancer as a traumatic event can decrease cancer HRQOL, and lead to poorer outcomes during cancer treatment. Most of this literature has been conducted in breast cancer samples. Given that lung cancer is often diagnosed at later stages, has a shorter disease trajectory and the likelihood of early mortality is significantly increased, investigating factors that may impact HRQOL for this population is essential. The literature also suggests that mindfulness not only serves to buffer (i.e. moderate) posttraumatic stress, but it can also foster resilience and facilitate (i.e. mediate) recovery and well-being following a cancer diagnosis.

The relationships between trauma, cancer HRQOL, and mindfulness have been studied independently of one other and have yet to be examined in a unified framework. Additionally, none of these relationships have been studied within the context of lung cancer. The diagnosis of lung cancer brings with it a unique set of challenges and stressors including internalized blame, stigma, and poor prognosis (Cataldo et al., 2011). Improving HRQOL for advanced cancer patients remains a primary treatment goal for any type of cancer, and is especially salient for lung cancer patients. Specifically, understanding how trauma fosters vulnerability to poor outcomes and how mindfulness promotes resilience can enable researchers and clinicians to better care for their lung cancer patients. 
The proposed study endeavors to take the next step in understanding the relationships between trauma, HRQOL and mindfulness in lung cancer patients. This exploratory cross-sectional study will examine the potential moderating role mindfulness may play within a lung cancer sample. Dispositional mindfulness may be conceptualized as a pre-existing factor that is similar to race and sex, which makes the timing of measurement for the assumptions needed for moderation less problematic. As this is a cross-sectional study, mediation will not be tested.

\section{Summary and Hypotheses}

Based on the reviewed literature, and the previously discussed models of how these variables may intersect, this dissertation will explore three specific aims: The first is to explore the relationship between trauma (as measured by frequency of traumatic events, traumatic distress appraisal and current cancer-specific distress) and HRQOL in a sample of lung cancer patients. The second aim is to examine the relationships of mindfulness with trauma and HRQOL. The third aim will explore the potential protective role mindfulness may play on trauma and HRQOL. 


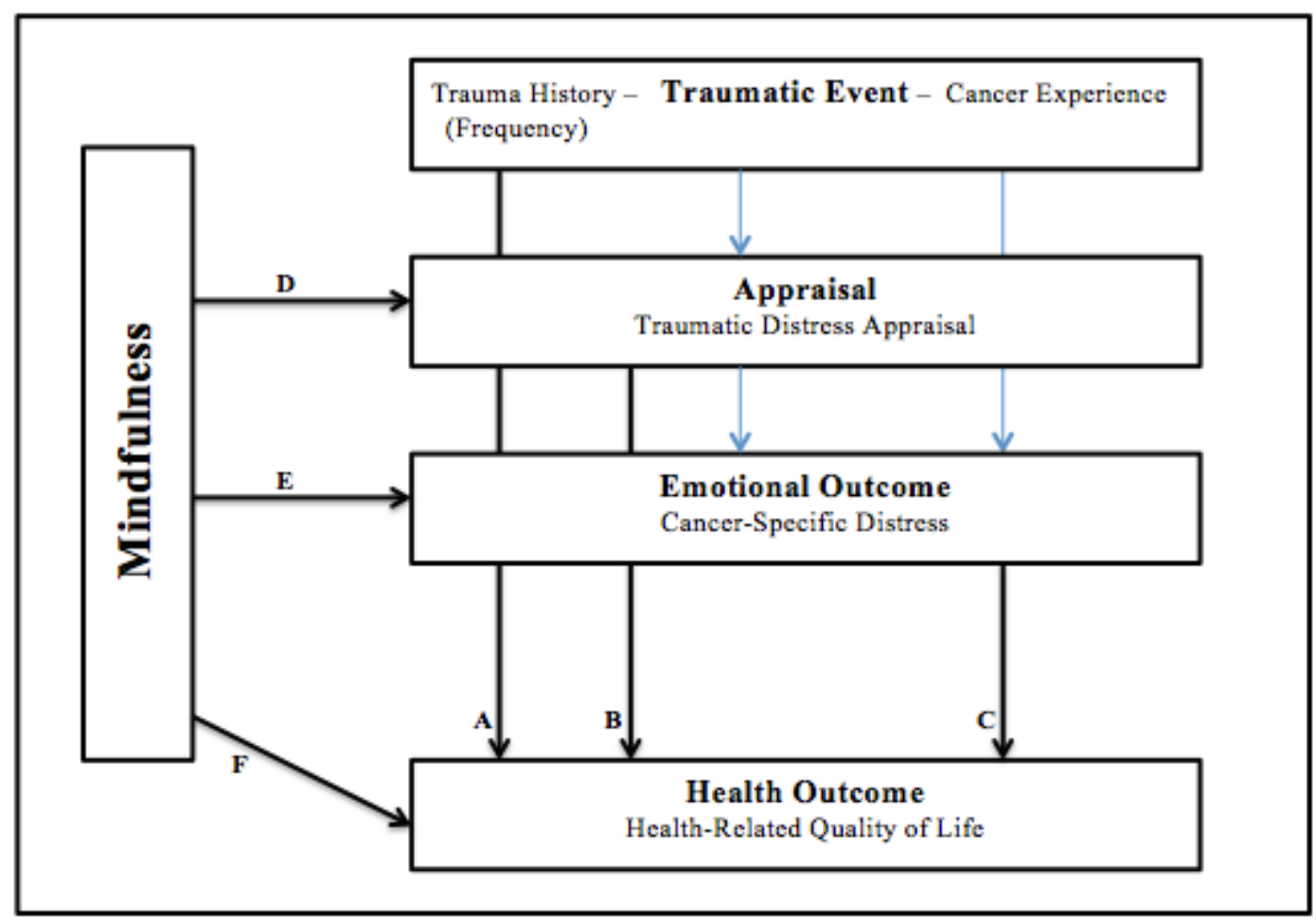

Figure 2. A conceptual model that proposes the potential pathways of intersection between variables of interest (Aims 1 and 2). The grey arrows will be explored in preliminary analyses.

Aim 1: Examine the relationship between trauma and HRQOL.

Hypothesis A. Frequency of traumatic events will be associated with poorer HRQOL (Figure 2, Arrow A).

Hypothesis B. Traumatic distress appraisal will be associated with poorer HRQOL (Figure 2, Arrow B).

Hypothesis $\boldsymbol{C}$. Cancer-specific distress will be associated with poorer HRQOL (Figure 2, Arrow C).

Aim 2: Examine the relationships of mindfulness with trauma and HRQOL.

Hypothesis D: Mindfulness will be associated with lower traumatic distress appraisal (Figure 2, Arrow D). 
Hypothesis E: Mindfulness will be associated with lower cancer-specific distress

(Figure 2, Arrow E).

Hypothesis F: Mindfulness will be associated with higher HRQOL (Figure 2, Arrow F).

Aim 3: Explore mindfulness as a protective factor that may buffer associations between trauma (as measured by traumatic distress appraisal and cancer-specific distress) and HRQOL.

Hypothesis $\boldsymbol{G}$. Mindfulness will moderate the effects of traumatic distress appraisal on HRQOL (Figure 3, Arrow G).

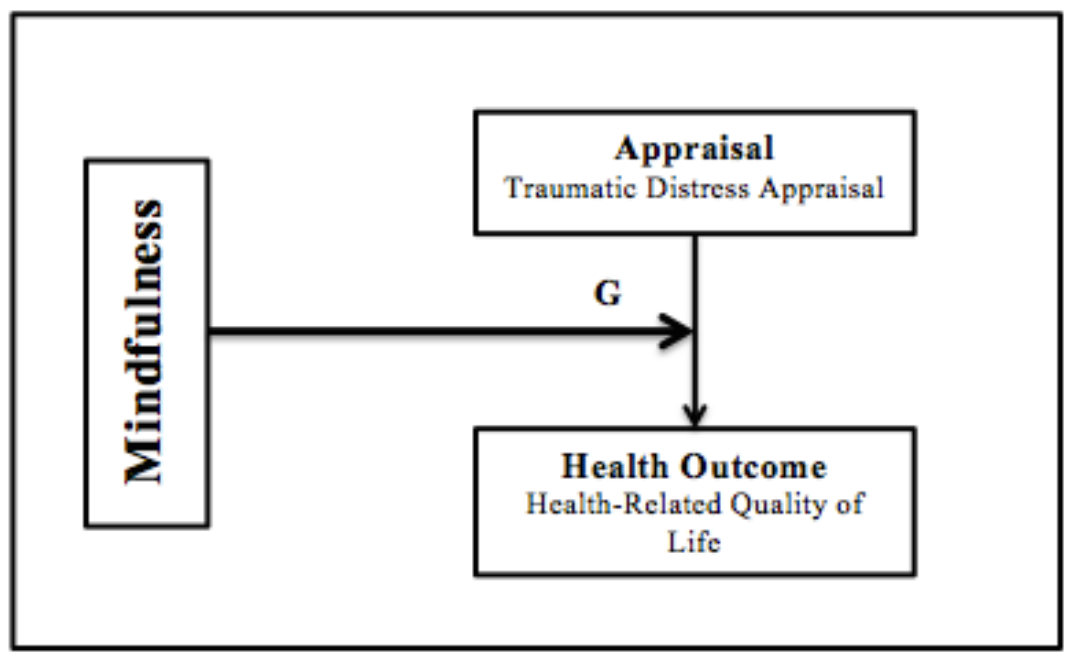

Figure 3: Mindfulness moderates traumatic distress appraisal on HRQOL.

Hypothesis $\boldsymbol{H}$. Mindfulness will moderate the effects of cancer-specific distress on HRQOL (Figure 4, Arrow H). 


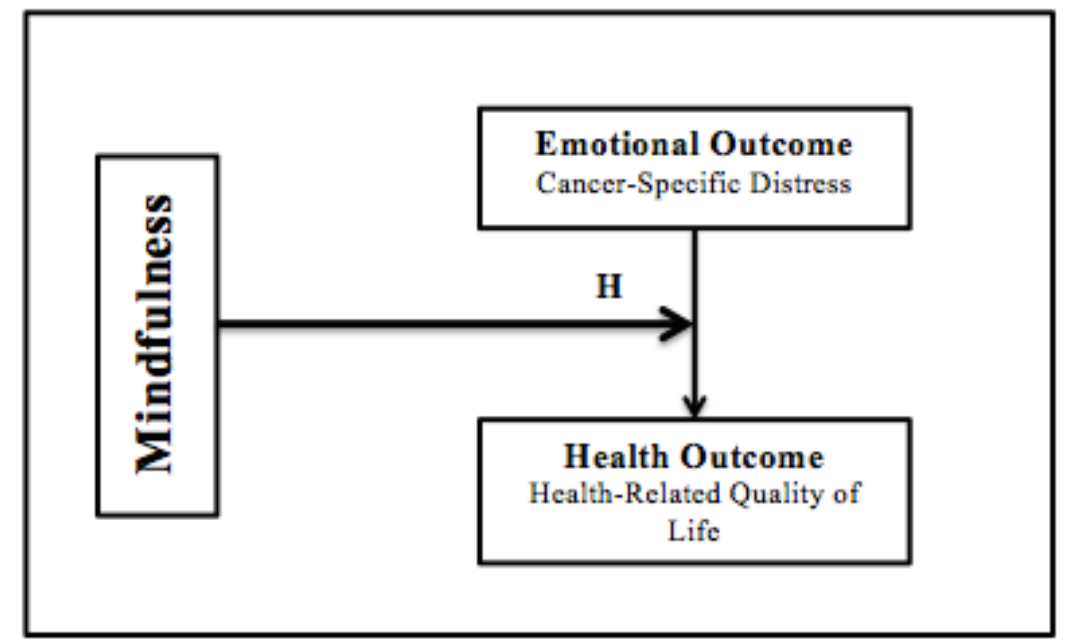

Figure 4: Mindfulness moderates cancer-specific distress on HRQOL. 


\section{METHOD}

\section{Recruitment}

All study personnel were trained by the University of Louisville to conduct research involving human subjects, and were trained by the project coordinator to conduct interviews in order to maintain a standardized interviewing procedure. Patients diagnosed with lung cancer were all recruited from the James Graham Brown Cancer Center in Louisville, KY, and were identified by accessing the electronic scheduling system utilized at these offices. Study personnel screened charts utilizing a screening checklist with eligibility criteria to verify if potential participants met inclusion/exclusion criteria. Research assistants actively built relationships with clinic staff in order to facilitate referrals to the study and to discuss potential issues as they arose. Eligible patients were referred to the study by the attending physician and met with study personnel at one of their clinic visits. Patients were given information regarding the study and requirements of participation. Patients were given the opportunity to have questions answered, and interested patients were provided with study materials.

\section{Participants}

A total of 51 participants were enrolled from February 2014 to April 2016. Initial study eligibility criteria included being between the ages of 18 and 85 , have received a diagnosis of non-small cell lung cancer within the previous five years, have no concurrent medical diagnosis likely to influence short-term (six-month) survival, live within a 120- 
mile radius of Louisville, KY, have no history of psychiatric hospitalization, no history of drug or alcohol abuse within the previous two months, and no known immune compromising conditions such as HIV/AIDS. Study personnel took information regarding cancer stage from the initial eligibility screening from the chart review. A brief interview of standard questions that assessed for exclusion criteria (concurrent medical diagnoses, history of psychiatric hospitalizations, history of recent drug or alcohol abuse, and assessment of immune compromising conditions) was completed at study enrollment.

A total of 433 patient charts were reviewed for eligibility to enter the study. Of the 433 patients screened, 273 patients were excluded for not meeting eligibility criteria. Of the 433 patients screened, research personnel identified 160 patients (40\%) being eligible for the study. Research personnel communicated with the physicians regarding eligible participants, and were invited to patient follow-up appointments at the Cancer Center. Of the 160 patients eligible for enrollment, 74 (46.3\%) were scheduled for appointments with their physician and study personnel to join the study, and 86 have yet to be contacted. As patients are recruited through their physicians at the time of an appointment, there are a large number of potentially eligible participants that have yet to have a clinical appointment with study personnel present.

Of the 74 eligible participants identified, nine participants were not approached as two had passed away before their recruitment appointments, and seven potential participants were not recommended for enrollment by their physician prior to meeting with study personnel. The physicians elaborated to study personnel that these seven eligible participants were no longer suitable for the study due to poor prognosis $(n=3)$ and recent concurrent medical diagnoses not yet noted in the chart that would make the 
study too burdensome $(n=4)$. Fourteen eligible participants refused participation; reasons for refusing participation in the study included believing the study too burdensome $(n=8)$, having memory deficits that would impact data collection $(n=2)$, and for personal reasons $(n=4)$. A total of 51 participants $(68.9 \%)$ agreed to participate and were enrolled.

\section{Data Collection Procedure}

Informed consent. This study was conducted in accordance with the University of Louisville Institutional Human Subjects Committee guidelines. All participants were given the opportunity to review forms, ask questions and were enrolled in the study signing informed consent and Health Information and Privacy Protection Act (HIPPA) documents approved by the University of Louisville Institutional Review Board on the use of Human Subjects in Research.

Procedure. Patients interested in participating were referred to study personnel by one of our referring physicians, and were scheduled for an interview with a research assistant. As part of a larger study, a large number of measures were collected that were not used for the purposes of this dissertation. This data collection included the following: after providing informed consent, participants were provided with an actigraph watch, a packet of materials and instructions for home-based saliva collection, and a short takehome questionnaire to complete (10 minutes per day over the subsequent 10 days). Wristworn actigraphs measured sleep and rest-activity rhythms, and waking and bedtime saliva samples were collected to measure cortisol over the 10-day baseline collection period. Additional data included a blood sample for serum measurement of cytokines and chemokines. Questionnaires measured demographic and psychosocial variables, and only 
the measures of demographics, medical variables, trauma, HRQOL and mindfulness were included for analyses in this dissertation. Upon completion of baseline data collection, participants scheduled a time for a research assistant to retrieve data at their convenience and were compensated with a $\$ 100$ pre-paid Visa card. Participants were also invited to take part in a meditation-based intervention, and to provide three-month follow-up data. For the purposes of this dissertation, only the baseline psychosocial variables were utilized in analyses.

\section{Measures}

Demographic and medical data. In order to account for potential effects of participant differences, several demographic variables were obtained as part of the selfreport questionnaire packet. These variables included date of birth, education, race/ethnicity, employment, gender and household income. Additionally, participants were asked to provide information on their date of diagnosis, current or past lung cancer treatments, and medications they are currently taking. Information regarding stage of cancer and date of diagnosis was confirmed through medical records review. In cases of discrepancy between participant report of stage of cancer and date of diagnosis, medical record data was utilized in all analyses.

Trauma History. The Trauma History Questionnaire (THQ) is a measure that examines experiences with potentially traumatic events such as crime, general disaster, life-threatening illness diagnosis, and sexual and physical assault (Green, 1996). The 24item THQ asks the participants to record the frequency of experiencing a potentially traumatic event in a yes/no format, the number of times it occurred and the approximate 
age(s) at the time of the event (Green, 1996). The test-retest reliability of reporting specific traumatic events ranged from .51 to .90 (Hooper et al., 2011) and the correlation for number of items endorsed across administrations was .70 (Green, 1996). This scale has been utilized among cancer populations (Green et al., 2000). As the measure looks at the frequency or presence of traumatic events, and not the subjective appraisal of traumatic distress, a Likert scale ranging from "not at all traumatic" to "extremely traumatic" was added to each item to provide additional characterizing information. Both the summary score of frequency of traumatic events and the traumatic distress appraisal score were utilized in primary analyses.

Cancer-Specific Distress. The Impact of Events Scale-Revised (IES-R) is a measure of a participant's current level of distress related to their cancer experience (Weiss \& Marmar, 1997). The IES-R measures, intrusive thoughts, avoidance and hyperarousal symptoms associated with the diagnosis and treatment of cancer. The 22item measure asks that participants rate the frequency of the occurrence of each item over the past seven days on a five-point Likert scale ranging from "not at all" to "extremely" (Weiss \& Marmar, 1997). The IES-R yields a total distress score and subscale scores can also be calculated for the intrusion, avoidance and hyperarousal subscales that correspond to the PTSD symptom clusters (Weiss \& Marmar, 1997). The internal consistency was .96 for the total score, .94 for the intrusion subscale, .87 for the avoidance subscale and .91 for the hyperarousal subscale in a sample of Vietnam veterans (Creamer, Bell, \& Failla, 2003). Scores greater than 22 for the IES-R total score are of high clinical concern in a sample of substance abusers (Rash, Coffey, Baschnagel, Drobes, \& Saladin, 2008); however, this is an arbitrary cutoff and does not indicate a specific clinical diagnosis 
(Rash et al. 2008). Scores of 33 may be clinically significant for the presence of symptoms of PTSD (Creamer et al. 2003). The total mean score of the IES-R was utilized for primary analyses. Subscale scores for the intrusion, avoidance and hyperarousal subscales were utilized for secondary analyses.

Cancer Health-Related Quality of Life. The Functional Assessment of Cancer Therapy-Lung (FACT-L; Cella et al., 1995) is a 44-item self-report instrument, which measures multidimensional quality of life among lung cancer patients. This measure yields a total score and 6 subscales: physical well-being, social/family well-being, relationship with doctor, emotional well-being, functional well-being and the lung cancer subscale. The lung cancer well-being subscale measured symptoms such as shortness of breath, losing weight, thinking clearly, coughing, hair loss, feeling tightness in the chest, breathing and smoking history. Each item uses a five-point Likert scale that ranges from "not at all" to "very much" (Cella et al., 1995). Test-retest reliability for the total score was reported as .92 (Cella et al., 1995). The FACT-L has also been proven as a reliable measurement of HRQOL for smokers with lung cancer in particular (Browning, Ferketich, Otterson, Reynolds, \& Wewers, 2009). The total mean score of the FACT-L was utilized for primary analyses. Five subscale scores (physical well-being, social/family well-being, emotional well-being, functional well-being and lung-cancer wellness) were utilized in secondary analyses.

Mindfulness. The Five-Facet Mindfulness Questionnaire (FFMQ; Baer, Smith, Hopkins, Krietemeyer, \& Toney, 2006) is a 39-item questionnaire that has five component skills which are theorized to contribute to mindfulness: (1) observing, (2) describing, (3) acting with awareness, (4) nonjudging of inner experiences, and (5) 
nonreactivity. Items are rated on a five-point Likert scale ranging from "never or very rarely true" to "very often or always true." The five facet scales show good internal consistency with alpha coefficients ranging from .75-.91 (Baer et al. 2006). The FFMQ appears to be a reliable measure of mindfulness as a multifaceted and dynamic construct (Curtiss \& Klemanski, 2014). The total mean score of the FFMQ was utilized for primary analyses. All five subscales were utilized in secondary analyses.

\section{Statistical Analyses}

Data Reduction and Missing Data. All data were cleaned before analyses were conducted. Two assistants entered questionnaire data into independent databases. The databases were compared to ensure correct entry and the files were merged to check for errors. If data were missing for fewer than half of items in a subscale, points were replaced using a mean replacement strategy of other responses on the subscale. Missing data was not replaced for the Traumatic History Questionnaire as each question is specific to a particular event, and each question does not correspond to a unifying underlying construct that would make a mean replacement strategy appropriate. Following mean replacement strategies for missing data, there were no more random instances of missing data. All variables were examined for normality and outliers prior to running analyses.

Control Variables. Bivariate correlations were used to examine the relationships between control variables, predictor variables and the dependent variable of HRQOL.

Confirmatory versus Exploratory Analyses. Based on a review of the literature, there is sufficient research in the literature to support Aims 1 and 2; thus these hypotheses were based on a confirmatory approach. This approach allows more definitive 
conclusions to be drawn in the event of a significant finding. Exploratory analyses were utilized for Aim 3 given the lack of research in this area.

Power. Utilizing the G*Power 3.1.3 (Erdfelder, Faul, \& Buchner, 1996) power analysis software, a power analysis was conducted for the proposed statistical analyses to determine the necessary sample size. In order to conduct multiple linear regressions to explore the relationships between trauma, HRQOL and mindfulness, an a priori power analysis was completed for each major aim. Aims 1 and 2 demonstrated medium to large effect sizes for hypothesized relationships in other samples. With $\alpha=.05$, power at $80 \%$, and the number of predictors of six, the power analysis estimates the sample size needed to have been between 13 and 46 participants. Analyses were conducted following the recruitment of the $51^{\text {st }}$ participant.

Sample Characteristics. Characteristics of the sample were determined by calculating frequency, percentage, and mean data on age, race/ethnicity, income, education, employment, age at diagnosis, and cancer stage.

Descriptive Data. Descriptive data in the form of means, standard deviations, and percentages within clinical ranges were calculated, outliers were identified and excluded, and summary variables were tested for normality. Bivariate correlations tested for collinearity.

Preliminary Analytical Procedures. The assumption of normal distribution of variables was evaluated by examining histograms, and the skewness statistic. Variables that were non-normally distributed were log-transformed. For variables that did not demonstrate normality after log transformation, histograms between transformed and original variables were compared. The more normally distributed histogram was selected 
for further analyses. For those that remained non-normal, Spearman correlations were used as opposed to Pearson correlations for subsequent bivariate correlations. Exploratory bivariate correlations were calculated to allow for examination of relationships between control variables and both independent and dependent variables. Associations between measures of traumatic events, traumatic distress appraisal and cancer specific distress (as indicated by the grey arrows in Figure 2) were also explored in bivariate correlations for potential collinearity. Prior to running multiple hierarchical regressions, the following regression assumptions were tested and met: (1) linear relationships, (2) multivariate normality, (3) no multicollinearity (4) homoscedasticity.

Aim 1: Examine the relationship between trauma and HRQOL. Hypotheses

A, B, and C: Hierarchical linear regressions were performed to determine the relationship between trauma and HRQOL. The theoretically-derived control variables, age at diagnosis, cancer stage, income and gender, were entered in the first block, trauma history (as measured by frequency of traumatic events (Arrow A), traumatic distress appraisal (Arrow B) and cancer specific distress; Arrow C) were entered in the second block and HRQOL was entered as the outcome. A total of three regressions were run to test this aim.

Aim 2: Examine the relationships of mindfulness with trauma and HRQOL.

Hypotheses D, E, and F: Hierarchical linear regressions were performed to explore the associations of mindfulness with trauma, cancer-specific distress, and HRQOL. The theoretically-derived control variables, age at diagnosis, cancer stage, income and gender, were entered in the first block, mindfulness was entered in the second block and 
traumatic distress appraisal (Arrow D), cancer specific distress (Arrow E), and HRQOL (Arrow F) were entered as outcomes in three separate regressions.

\section{Aim 3: Explore the potential protective role of mindfulness with regard to} associations between trauma and HRQOL (Hypotheses $\mathbf{G}$ and $H$ ). In exploratory analyses that are meant to inform future hypotheses, tests of moderation were proposed. As represented in Figures 3 and 4, these hypotheses propose that mindfulness acts as a moderator in the association between traumatic distress appraisal and HRQOL, and between cancer specific distress and HRQOL. By considering both predictors and the dependent variable as continuous variables (Baron \& Kenny, 1986), the moderation is predicted to occur such that the interaction between mindfulness and traumatic distress appraisal is associated with HRQOL. To run a test of moderation, several assumptions must be met. First, the potential moderating variable (dispositional mindfulness) can not be significantly correlated with either the individual predictor (traumatic distress appraisal or cancer-specific distress) or the dependent variable (HRQOL; Baron \& Kenny, 1986; Kraemer, Stice, Kazdin, Offord, \& Kupfer, 2001). If this initial assumption was met, the following assumptions were also examined prior to conducting analyses: (1) homogeneity of variance, (2) temporal precedence of the moderating variable, (3) independence of error measurement (Baron \& Kenny, 1986; Fairchild \& MacKinnon, 2009; Kraemer et al., 2001).

Following an exploration of all assumptions, trauma (as measured by both traumatic distress appraisal and cancer specific distress) and mindfulness were centered and an interaction term was calculated (to eliminate multicollinearity). Hierarchical linear regressions were performed to determine the relationship between the interaction term 
and HRQOL. The theoretically-derived control variables, age at diagnosis, cancer stage, income and gender, were entered in the first block, centered trauma, centered mindfulness and the interaction term of trauma and mindfulness were entered in the second block and HRQOL was entered as the outcome. A total of two regressions were run to test this hypothesis.

Secondary analyses. For Aims 1 and 2 (hypotheses A-F), secondary analyses were conducted if the primary hypotheses were significant. Subscales were explored to further elucidate the significant findings. Hierarchical linear regressions were performed to determine the relationships between subscales and overall summary scores for significant findings. The theoretically-derived control variables, age at diagnosis, cancer stage, income and gender were entered in the first block of every regression and subscales and summary scores were utilized as either the predictor or dependent variables as appropriate. 


\section{RESULTS}

\section{Sample Characteristics}

A total of 51 participants were recruited for this study. Two participants were excluded from analyses: one refused to return study materials following enrollment, and the other did not meet eligibility criteria upon further review (diagnosed more than 5 years prior to enrollment). An additional three participants were excluded from analyses due to incomplete data. Hence, a total of 46 participants $(90.2 \%)$ provided complete data. Study participants ranged in age from 44 to 81 years. Most of the sample was female (N $=34,73.9 \%)$ and 12 participants $(26.1 \%)$ were male. The majority of the sample $(\mathrm{N}=35$, 72.9\%) was European American and 57.8\% $(\mathrm{N}=26)$ had fewer than twelve years of education. Additional socio-demographic characteristics of the sample are presented in Table 1.

\section{Table 1}

Demographic characteristics of the sample

\begin{tabular}{lcc}
\hline \multicolumn{1}{c}{ Variable } & Frequency & Percentage \\
\hline Race & & \\
Black or African American & 10 & $21.7 \%$ \\
European American & 35 & $76.1 \%$ \\
Hispanic/Latino & 1 & $2.2 \%$ \\
Education & & \\
High School or Less & 26 & $57.8 \%$ \\
AA/Technical & 12 & $23.7 \%$ \\
College Degree & 4 & $8.9 \%$ \\
Master's Degree & 3 & $6.7 \%$ \\
Gender & & \\
Male & 12 & $26.1 \%$ \\
\hline
\end{tabular}




\begin{tabular}{lcc}
\hline Female & 34 & $73.9 \%$ \\
$\quad$ Post-Menopausal & 19 & $38.8 \%$ \\
Income & & \\
$\quad$ Less than $\$ 20,000$ & 15 & $30.6 \%$ \\
$\$ 20,000-\$ 39,999$ & 12 & $24.5 \%$ \\
$\$ 40,000-\$ 59,000$ & 9 & $18.4 \%$ \\
$\$ 60,000-\$ 79,999$ & 6 & $12.2 \%$ \\
$\$ 80,000-\$ 99,999$ & 5 & $4.1 \%$ \\
$\quad \$ 100,000$ and above & $59.6(9.4)$ & $10.2 \%$ \\
Age at Diagnosis & $61.5(9.14)$ & \\
Mean (SD) & & \\
Age at Study Enrollment & $21.7(15.0)$ & \\
Mean (SD) & & \\
Time Since Diagnosis (Months) & & \\
Mean (SD) &
\end{tabular}

Of note, this particular sample is younger than the national average age of incident lung cancer cases. According to the American Cancer Society (2016), lung cancer often impacts older adults nationwide, with an average age of 70 at the time of diagnosis. Table 2 displays clinical characteristics of the sample. The majority of the sample (67\%) was diagnosed in later stages of lung cancer (stages III or IV), and 33\% of the sample was diagnosed in early stages of cancer (stages I or II). Despite meeting eligibility criteria that aimed to exclude cases with medical conditions likely to influence six-month survival, $10.2 \%$ of participants expired after providing baseline data.

Table 2

\section{Clinical Characteristics}

\begin{tabular}{lcc}
\hline \multicolumn{1}{c}{ Variable } & Frequency & Percentage \\
\hline Stage & 1 & \\
Stage I & 1 & $2 \%$ \\
Stage IA & 6 & $12.2 \%$ \\
Stage IB & 4 & $8.2 \%$ \\
Stage IIA & 1 & $2 \%$ \\
Stage IIB & 4 & $8.2 \%$ \\
Stage IIIA & 16 & $32.7 \%$ \\
Stage IIIB & 3 & $6.1 \%$ \\
Stage IV & 12 & $24.5 \%$ \\
\hline
\end{tabular}




\begin{tabular}{lcc}
\hline Stage IVA & 2 & $4.1 \%$ \\
Comorbid medical & 32 & $66.7 \%$ \\
conditions & 13 & $28.3 \%$ \\
$\quad$ High Blood Pressure & 9 & $19.6 \%$ \\
Diabetes & 4 & $8.7 \%$ \\
Atrial Fibrillation & 3 & $6.5 \%$ \\
COPD & 3 & $6.5 \%$ \\
Emphysema & 44 & $95.7 \%$ \\
Smoking History & & \\
$\quad$ Ever Smoked in Their & 2 & \\
Lifetime & & \\
Currently Smoking & $46.1(10.9)$ & \\
Number of years smoking & & \\
Mean (SD) & $837.4(633.0)$ & \\
Number of Pack Years & & \\
Mean (SD) &
\end{tabular}

Table 3

Descriptive statistics for variables of trauma, HRQOL and mindfulness

\begin{tabular}{lccc}
\hline \multicolumn{1}{c}{ Variable } & N & Mean & Std. Deviation \\
\hline $\begin{array}{l}\text { Frequency of Traumatic } \\
\text { Events (THQ - Number of } \\
\text { Traumatic Events Over the }\end{array}$ & 47 & 5.85 & 3.58 \\
Lifetime) & & & \\
$\quad$ Crime-related & 47 & .872 & 1.03 \\
$\quad$ General Disaster & 47 & 4.04 & 2.38 \\
$\quad$ Physical and Sexual Assault & 47 & .936 & 1.33 \\
Traumatic Distress Appraisal & 44 & 31.41 & 16.84 \\
(THQ) & & & \\
Cancer-Specific Distress & 46 & 20.49 & 17.39 \\
(IES-R) & & & \\
$\quad$ Intrusions & 46 & .979 & .817 \\
$\quad$ Hyperarousal & 46 & .771 & .797 \\
$\quad$ Avoidance & 46 & 1.00 & .901 \\
Health Related Quality of & 46 & 88.96 & 20.70 \\
Life (FACT-L) & & \\
$\quad$ Physical Well-Being & 46 & 18.50 & 4.28 \\
$\quad$ Social and Family Well- & 46 & 18.07 & 5.96 \\
Being & & & \\
$\quad$ Emotional Well-Being & 46 & 17.23 & 5.19 \\
$\quad$ Functional Well-Being & 46 & 17.21 & 6.54 \\
$\quad$ Lung Cancer Wellness & 46 & 17.96 & 5.39 \\
Mindfulness (FFMQ) & 47 & 129.57 & 18.82 \\
$\quad$ Observe & 47 & 24.77 & 6.11 \\
\hline
\end{tabular}




\begin{tabular}{llll}
\hline Describe & 47 & 25.34 & 6.04 \\
Act with Awareness & 47 & 28.60 & 6.32 \\
Nonjudgment & 47 & 30.15 & 6.17 \\
Nonreactivity & 47 & 20.71 & 5.22 \\
\hline
\end{tabular}

Frequency of Traumatic Events and Traumatic Distress Appraisal. The mean number of traumatic events was $5.85(\mathrm{SD}=3.58)$ endorsed over the lifetime. The majority of the sample (98.8\%) endorsed having experienced at least one traumatic event in their lifetime; most commonly three different traumatic events were endorsed $(21.3 \%$ of the sample). The mean score for the overall traumatic distress appraisal of this sample was $31.41(\mathrm{SD}=16.84)$.

The most frequently reported type of traumatic event was General Disaster related traumatic events. This category included potentially traumatic events such as serious accidents, experiencing a natural or man-made disaster, serious injury, a situation that might cause death or injury, seeing death of a loved one, having a serious life-threatening illness, and engaging in combat. The most common type of traumatic event reported was having a serious life-threatening illness, with $80.9 \%$ of the sample reporting their lung cancer as a traumatic event and indicating that it was highly traumatic $(\mathrm{M}=6.07, \mathrm{SD}=$ $1.13)$.

Cancer-Specific Distress. Among this sample, overall cancer-specific distress (M $=20.49, \mathrm{SD}=17.39)$ was comparable to a sample of breast cancer patients $(\mathrm{M}=24.7$, $\mathrm{SD}=10.6$; Akechi et al., 2007). Total scores of $\geq 22$ (Rash et al., 2008) and $\geq 33$

(Creamer et al., 2003) were indicated as being of clinical significance. Figure 5 shows the distribution of total cancer-specific distress scores, with $39 \%$ of the sample $(\mathrm{N}=18)$ having scores $\geq 22$ and $28.3 \%$ of the sample $(\mathrm{N}=13)$ having scores $\geq 33$. 


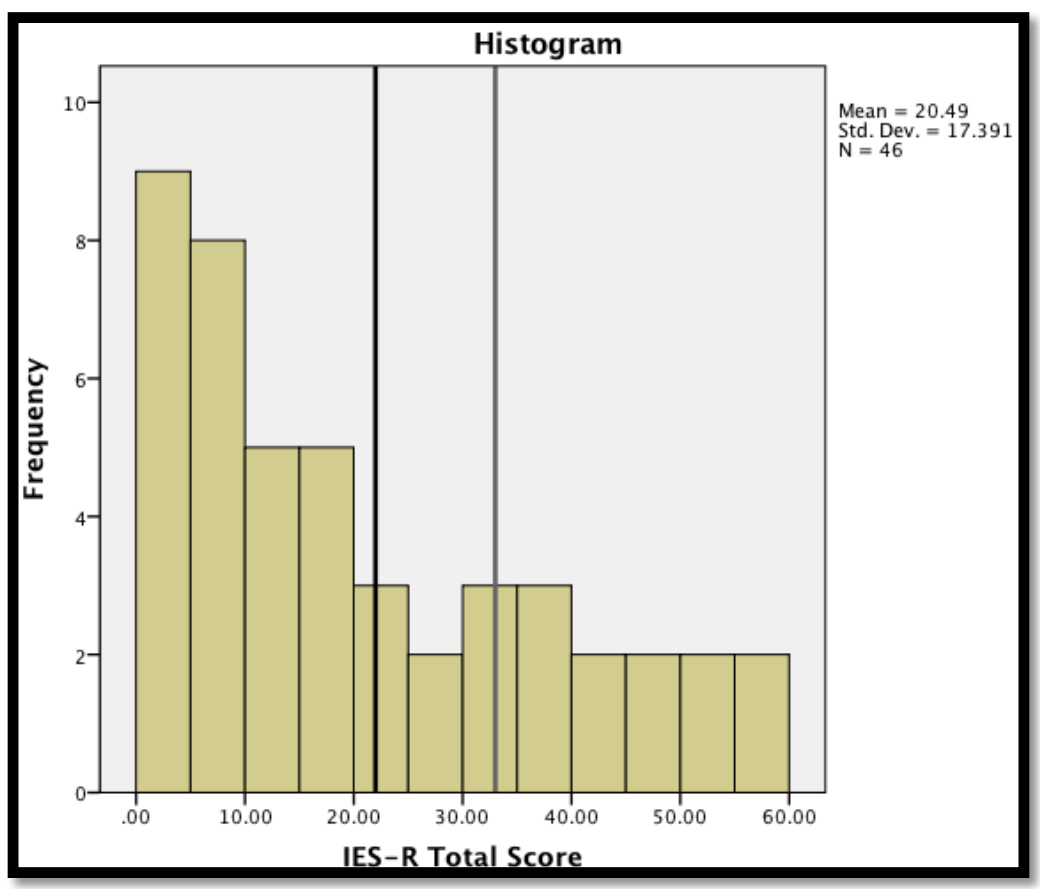

Figure 5. Histogram of clinical cutoff scores for the IES-R; The black line denotes the clinically significant cutoff score of 22; The gray line denotes the clinically significant cutoff score of 33 .

In comparison to a sample of prostate cancer patients (Banthia et al., 2003), the IES-R subscales had the following comparisons: (1) the intrusion subscale $(\mathrm{M}=.979, \mathrm{SD}$ $=.817)$ was higher than the prostate cancer sample $(\mathrm{M}=.83, \mathrm{SD}=.72) ;(2)$ the hyperarousal subscale $(\mathrm{M}=.771, \mathrm{SD}=.797)$ was much higher than the sample of prostate cancer patients $(\mathrm{M}=.47, \mathrm{SD}=.54)$, and $(3)$ the avoidance subscale $(\mathrm{M}=1.00$, $\mathrm{SD}=.901)$ was higher than the prostate cancer sample $(\mathrm{M}=.91, \mathrm{SD}=.82)$.

Health-Related Quality of Life. The mean score for the overall HRQOL of this sample $(M=88.96, S D=20.70)$ was comparable to a sample of lung cancer patients under the age of $65(\mathrm{M}=91.89, \mathrm{SD}=16.55$; Nipp et al., 2016). Comparing the mean subscale scores with other lung cancer patients (Cella et al., 1995) showed the following comparisons: (1) the physical well-being subscale $(\mathrm{M}=18.5, \mathrm{SD}=4.28)$ was slightly 
lower than the normative lung cancer sample $(\mathrm{M}=20.7, \mathrm{SD}=5.0)$; (2) the social and family well-being subscale $(\mathrm{M}=18.07, \mathrm{SD}=5.96)$ was lower than the normative lung cancer sample $(\mathrm{M}=23.2, \mathrm{SD}=3.9)$; (3) the emotional well-being subscale $(\mathrm{M}=17.23$, $\mathrm{SD}=5.19)$ was slightly higher than the normative lung cancer sample $(\mathrm{M}=15.5, \mathrm{SD}=$ 3.7); (4) the functional subscale $(\mathrm{M}=17.21, \mathrm{SD}=6.54)$ was comparable to the normative lung cancer sample $(\mathrm{M}=17.4, \mathrm{SD}=6.2)$; and $(5)$ the lung cancer subscale $(\mathrm{M}=17.96$, $\mathrm{SD}=5.39)$ was slightly lower than the normative lung cancer sample $(\mathrm{M}=20.5, \mathrm{SD}=$ 4.8).

Mindfulness. The mean score on the measure of overall mindfulness $(\mathrm{M}=$ 129.57, $\mathrm{SD}=18.82)$ was comparable to a healthy adult sample $(\mathrm{M}=121.6, \mathrm{SD}=20.31$; Robins, Keng, Ekblad, \& Brantley, 2012) as well as a sample of breast cancer patients (M $=132.32 \mathrm{SD}=4.54$; Matchim, Armer, $\&$ Stewart, 2010). Comparing the mean subscale scores with a heterogeneous cancer sample (breast, blood, colon, prostate, gynecoligc, other; Garland, Tamagawa, Todd, Speca, \& Carlson, 2013) showed the following comparisons: (1) the observe subscale $(\mathrm{M}=25.77, \mathrm{SD}=6.11)$ was slightly higher than the heterogeneous cancer sample $(M=23.31, S D=5.03)$; (2) the describing subscale $(M$ $=25.34, \mathrm{SD}=6.04)$ was comparable to the heterogeneous cancer sample $(\mathrm{M}=25.47, \mathrm{SD}$ $=5.82)$; (3) the act with awareness subscale $(\mathrm{M}=28.60, \mathrm{SD}=6.32)$ was slightly higher than the heterogeneous cancer sample $(\mathrm{M}=26.14, \mathrm{SD}=5.55)$; (4) the nonjudging subscale $(\mathrm{M}=30.15, \mathrm{SD}=6.17)$ was slightly higher than the heterogeneous cancer sample $(M=27.39, \mathrm{SD}=5.90)$; and (5) the nonreactivity subscale $(M=20.71, \quad \mathrm{SD}=$ 5.22) was comparable to the heterogeneous cancer sample $(\mathrm{M}=20.5, \mathrm{SD}=4.8)$.

\section{Preliminary Analyses}


Prior to running correlations and multiple linear regressions, assumptions for the proposed statistics were examined. To test for linearity and homoscedasticity, scatter plots were examined for outliers. There were no significant outliers noted in the scatter plots. The skewness statistic and visual examination of histograms were used to test the assumption of normality. For sample sizes less than 50 participants, a skewness statistic greater than 1.96 is considered skewed (Kim, 2013). The following variables involved in primary and secondary analyses were not normally distributed: total cancer-specific distress score $(I E S-R ;$ z-skew $=1.99)$, the total mindfulness score $(F F M Q ; z-s k e w=$ $2.99)$, total frequency of traumatic events $(\mathrm{THQ} ; \mathrm{z}-\mathrm{skew}=2.17)$, the nonreactivity subscale of the FFMQ (z-skew $=2.57)$, the hyperarousal $(\mathrm{z}$-skew $=2.44)$ and avoidance $(z$-skew $=2.57)$ subscales of the IES-R, and the physical well-being $(z$-skew $=2.02)$ and emotional well-being $(z-s k e w=1.98)$ subscales of the HRQOL. These variables were log-transformed and re-examined. All variables demonstrated a more normalized distribution following log-transformation with the exception of total cancer-specific distress $($ transformed $z$ - skew $=2.43)$, total mindfulness score $($ transformed $z$-skew $=$ 2.12), the physical well-being (transformed z-skew $=3.34$ ) and the emotional well-being subscales (transformed z-skew $=3.66$ ) of the HRQOL.

For variables that remained non-normally distributed, the histograms of both the transformed and original variables were visually compared. The more normally distributed variable, based on the visual examination of the histogram, was selected for analyses. Therefore, the following variables were transformed for final analyses: the total cancer-specific distress score, the total frequency of traumatic events, the total mindfulness score, the nonreactivity subscale of the FFMQ, and the hyperarousal and 
avoidance subscales of the IES-R. The original physical and emotional well-being subscales of the HRQOL were utilized in subsequent secondary analyses. The assumption of multicollinearity was examined by checking the correlations between all variables (see Table 4).

Pearson bivariate correlations were conducted to examine HRQOL with all predictor variables. For variables that remained non-normal, Spearman's rho was used. Each predictor variable demonstrated statistically significant associations with the dependent variable hypothesized with the exception of frequency of traumatic events and HRQOL (see Table 4).

\section{Table 4}

Bivariate Pearson correlations between independent and dependent variables.

\begin{tabular}{lcccc}
\hline \multicolumn{1}{c}{ Variable } & $\begin{array}{c}\text { Traumatic } \\
\text { Distress } \\
\text { Appraisal } \\
\text { (THQ) }\end{array}$ & $\begin{array}{l}\text { Cancer- } \\
\text { Specific } \\
\text { Distress } \\
\text { (IES-R) }\end{array}$ & $\begin{array}{c}\text { Mindfulness } \\
\text { (FFMQ) }\end{array}$ & $\begin{array}{c}\text { Health- } \\
\text { Related } \\
\text { Quality of } \\
\text { Life }\end{array}$ \\
\hline $\begin{array}{l}\text { Frequency of } \\
\text { Traumatic Events }\end{array}$ & $.896^{* *}$ & $\mathbf{- . 0 8 0}$ & $\mathbf{. 0 1 0}$ & -.175 \\
$\begin{array}{l}\text { (THQ) } \\
\text { Traumatic Distress }\end{array}$ & & & & \\
$\begin{array}{l}\text { Appraisal (THQ) } \\
\text { Cancer-Specific }\end{array}$ & & .122 & $\mathbf{- . 1 3 0}$ & $-.404 *$ \\
$\begin{array}{l}\text { Distress (IES-R) } \\
\text { Mindfulness (FFMQ) }\end{array}$ & & & $-.469 * *$ & $\mathbf{- . 5 6 2 * *}$ \\
\hline
\end{tabular}

$* \mathrm{p}<.05, * * \mathrm{p}<.01$; Note that correlations in bold are Spearman correlations

Control Variables. Control variables (age at diagnosis, cancer stage, gender and income) were included in order to increase generalizability to other lung cancer samples (Babyak, 2004). All theoretically derived control variables were included in subsequent analyses, regardless of statistically significant relationships with the outcomes of interest 
(see Table 5). Age at diagnosis (Linden, Vodermaier, MacKenzie, \& Greig, 2012) and cancer stage (Iwatani, Matsuda, Kawabata, Miura, \& Matsushima, 2013) were included due to their likely associations with predictor and outcome variables. Gender was selected as a theoretically derived covariate as it has been shown to be strongly associated with both predictors and HRQOL in lung cancer samples (Chang et al., 2015; Nipp et al., 2016). Annual household income was chosen as it has been shown to be associated with trauma and HRQOL in both breast and prostate cancer samples (Penson et al., 2001). Bivariate Spearman correlations were conducted among the predictor, control and outcome variables (Table 5). Regression models were held to a maximum of seven variables in order to help reduce the probability of making a Type 1 error.

\section{Table 5}

$\underline{\text { Bivariate Spearman correlations between control variables and predictor and outcome }}$ variables of interest.

\begin{tabular}{lcclcc}
\hline Variable & $\begin{array}{c}\text { Frequency } \\
\text { of } \\
\text { Traumatic } \\
\text { Events } \\
\text { (THQ) }\end{array}$ & $\begin{array}{c}\text { Traumatic } \\
\text { Distress } \\
\text { Appraisal } \\
\text { (THQ) }\end{array}$ & $\begin{array}{c}\text { Cancer- } \\
\text { Specific } \\
\text { Distress } \\
\text { (IES-R) }\end{array}$ & $\begin{array}{c}\text { Mindfulness } \\
\text { (FFMQ) }\end{array}$ & $\begin{array}{c}\text { Health- } \\
\text { Related } \\
\text { Quality of } \\
\text { Life }\end{array}$ \\
\hline Age at Diagnosis & -.076 & -.138 & -.177 & .151 & -.163 \\
Cancer Stage & -.057 & -.136 & .021 & $.383 * *$ & .088 \\
Gender & .083 & .182 & .033 & .035 & -.006 \\
Annual Household & -.005 & -.006 & .035 & .050 & .067 \\
Income & & & & &
\end{tabular}

\section{Primary Analyses}

\section{Hypothesis A: frequency of traumatic events will be associated with poorer}

HRQOL (Figure 2, Arrow A). Bivariate correlations revealed that frequency of traumatic events was not significantly associated with HRQOL. Subsequent hierarchical 
regression analyses controlled for age at diagnosis, cancer stage, gender and income (see Table 6). In all analyses, control variables entered in the first step did not add significant explanatory power to the model, nor did the control variables significantly predict HRQOL. The addition of frequency of traumatic events did not add significant explanatory power to the model (partial $\mathrm{r}=-.169, \mathrm{p}=.309$ ), and the model remained nonsignificant. Overall frequency of traumatic events in lung cancer patient's history was not associated with decrements in HRQOL.

\section{Table 6}

Summary of hierarchical regression analyses entering Frequency of Traumatic Events as the predictor of HRQOL (FACT-L, N = 41)

\begin{tabular}{|c|c|c|c|c|c|c|c|c|}
\hline Variabl & & $\mathrm{N}$ & B & SE B & $\beta$ & $\mathrm{R}^{2}$ & $\Delta \mathrm{R}^{2}$ & $\mathrm{p}$ of $\Delta \mathrm{R}^{2}$ \\
\hline \multirow{5}{*}{ Step 1} & & & & & & .116 & .116 & .321 \\
\hline & Age at Diagnosis & & .766 & .358 & .340 & & & \\
\hline & Stage & & 1.641 & 3.333 & .082 & & & \\
\hline & Gender & & 3.802 & 7.736 & .082 & & & \\
\hline & Income & 41 & .652 & 1.004 & .105 & & & \\
\hline \multirow{2}{*}{ Step 2} & & & & & & .141 & .025 & .309 \\
\hline & $\begin{array}{l}\text { Frequency of Traumatic } \\
\text { Events }\end{array}$ & & -13.672 & 13.261 & -.160 & & & \\
\hline
\end{tabular}

Hypothesis B: Traumatic distress appraisal will be associated with poorer

HRQOL (Figure 2, Arrow B). The hypothesis that higher traumatic distress appraisal would be related to poorer HRQOL was tested by performing bivariate correlations followed by hierarchical multiple regressions controlling for relevant variables. In all analyses, control variables entered in the first step did not add significant explanatory power to the model. Bivariate correlations revealed higher traumatic distress was negatively associated with HRQOL $(\mathrm{r}=-.347, \mathrm{p}<.05$; see Table 4 for bivariate 
correlations). This result maintained significance even after entering four control variables. Control variables, included age at diagnosis, stage of cancer, gender and income, were entered in the first block and traumatic distress appraisal was entered in the second block. A multiple regression was preformed utilizing HRQOL as the dependent variable and relevant control variables and traumatic distress appraisal as predictors in order to determine if HRQOL scores were associated with traumatic distress appraisal.

The overall model was statistically significant $\left(\Delta \mathrm{R}^{2}=.139 ; \mathrm{F}(5,34)=2.83, \mathrm{p}=\right.$ .017). Traumatic distress appraisal was a significant individual predictor $(\beta=-.397, \mathrm{p}=$ .017 ; see Table 7). As indexed by the squared partial correlation coefficient, traumatic distress appraisal accounted for $15.7 \%$ of the total variability in HRQOL, which is considered a medium effect. The addition of traumatic distress appraisal added significant explanatory power to the model and predicted lower HRQOL.

\section{Table 7}

$\underline{\text { Summary of hierarchical regression analyses entering traumatic distress appraisal as the }}$ predictor of HRQOL (FACT-L, N = 39)

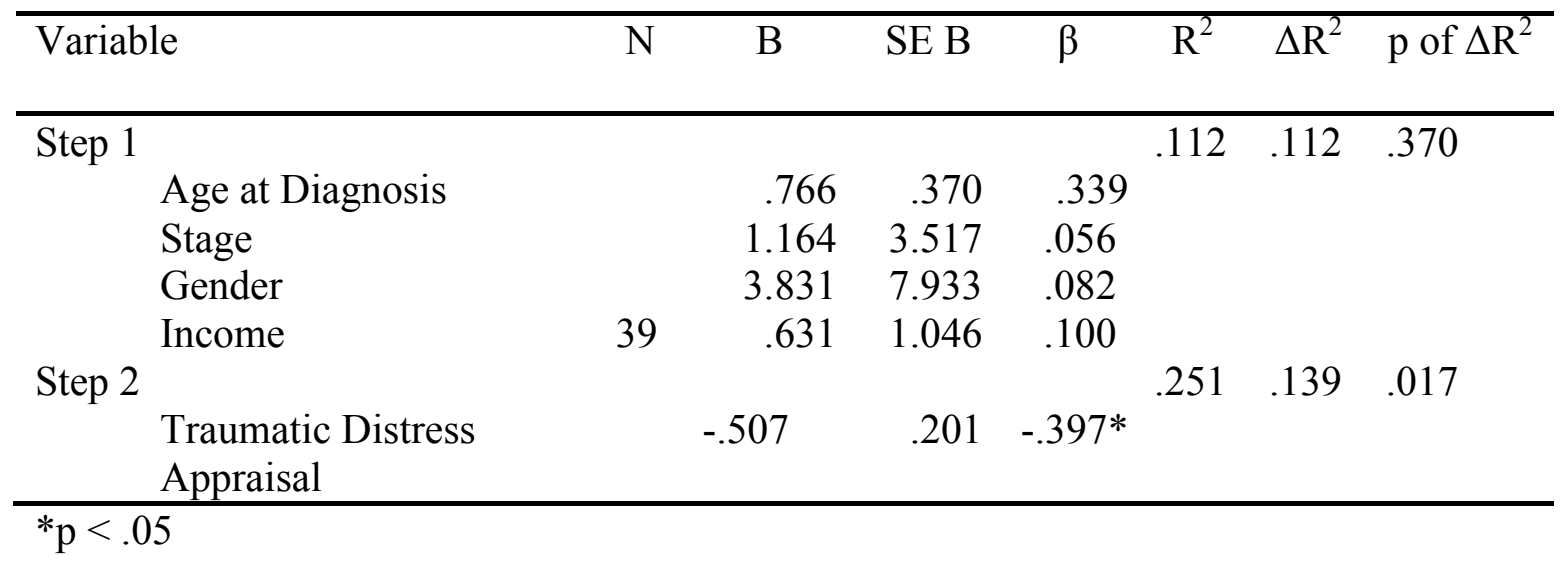




\section{Hypothesis C: Cancer-specific distress will be associated with poorer}

HRQOL (Figure 2, Arrow C). The hypothesis that higher cancer-specific distress would be related to poorer HRQOL was tested by performing bivariate correlations followed by hierarchical multiple regressions controlling for relevant variables. In all analyses, control variables entered in the first step did not add significant explanatory power to the model. Bivariate correlations revealed higher cancer-specific distress was negatively associated with HRQOL $(\mathrm{r}=-.562, \mathrm{p}<.01$; see Table 4 for bivariate correlations $)$. This result maintained significance even after entering four control variables. A multiple linear regression was preformed utilizing HRQOL as the dependent variable and relevant control variables and cancer-specific distress as predictors in order to determine if HRQOL scores could be predicted as a function of cancer-specific distress.

The overall model was statistically significant $\left(\Delta \mathrm{R}^{2}=.288 ; \mathrm{F}(5,35)=5.109, \mathrm{p}=\right.$ $.0002)$. Cancer-specific distress was a significant individual predictor $(\beta=-.562, \mathrm{p}=$ .0002; see Table 8). As indexed by the squared partial correlation coefficient, cancerspecific distress accounted for $33.3 \%$ of the total variability in HRQOL, indicating a large effect. The addition of cancer-specific distress added significant explanatory power to the model and predicted lower HRQOL.

\section{Table 8}

Summary of hierarchical regression analyses entering cancer-specific distress as the predictor of HRQOL (FACT-L, N = 39)

\begin{tabular}{|c|c|c|c|c|c|c|c|}
\hline Variable & $\mathrm{N}$ & $\mathrm{B}$ & SE B & $\beta$ & $\overline{\mathrm{R}^{2}}$ & $\Delta \mathrm{R}^{2}$ & $\begin{array}{l}\mathrm{p} \text { of } \\
\Delta \mathrm{R}^{2}\end{array}$ \\
\hline Step 1 & & & & & .134 & .134 & .256 \\
\hline Age at Diagnosis & & .815 & .351 & .370 & & & \\
\hline Stage & & 1.250 & 3.262 & .064 & & & \\
\hline
\end{tabular}




\begin{tabular}{|c|c|c|c|c|c|c|c|c|}
\hline Variab & & $\mathrm{N}$ & B & SE B & $\beta$ & $\overline{\mathrm{R}^{2}}$ & $\Delta \mathrm{R}^{2}$ & $\begin{array}{l}\mathrm{p} \text { of } \\
\Delta \mathrm{R}^{2}\end{array}$ \\
\hline \multirow{4}{*}{ Step 2} & Gender & & 4.799 & 7.578 & .106 & & & \\
\hline & Income & 39 & .614 & .981 & .102 & \multirow{3}{*}{422} & \multirow{3}{*}{288} & \multirow{3}{*}{.0002} \\
\hline & & & & & & & & \\
\hline & Cancer-Specific Distress & & -23.966 & 5.739 & $-.562 * *$ & & & \\
\hline
\end{tabular}

${ }^{*} \mathrm{p}<.05,{ }^{* *} \mathrm{p}<.01$

\section{Hypothesis D: Mindfulness will be associated with lower traumatic distress}

appraisal (Figure 2, Arrow D). Bivariate correlations revealed that increased

mindfulness was not significantly associated with traumatic distress appraisal (Table 4).

Subsequent hierarchical regression analyses controlled for age at diagnosis, cancer stage, gender and income (see Table 9). In all analyses, control variables entered in the first step did not add significant explanatory power to the model, nor did the control variables significantly predict traumatic distress appraisal. The addition of mindfulness did not add significant explanatory power to the model $\left(\Delta \mathrm{R}^{2}=.011 ; \mathrm{F}(5,34)=.130, \mathrm{p}=.985\right)$, and the model remained not significant. Overall, mindfulness was not significantly associated with lower traumatic distress appraisal.

\section{Table 9}

$\underline{\text { Summary of hierarchical regression analyses entering mindfulness as the predictor of }}$ traumatic distress appraisal (THQ-Appraisal, $\mathrm{N}=39$ )

\begin{tabular}{|c|c|c|c|c|c|c|c|c|}
\hline \multicolumn{2}{|c|}{ Variable } & $\mathrm{N}$ & B & SE B & $\beta$ & $\overline{\mathrm{R}^{2}}$ & $\overline{\Delta \mathrm{R}^{2}}$ & $\mathrm{p}$ of $\Delta \mathrm{R}^{2}$ \\
\hline \multirow{5}{*}{ Step 1} & & & & & & .116 & .116 & .349 \\
\hline & Age at Diagnosis & & -.269 & 290 & -.152 & & & \\
\hline & Stage & & -3.923 & 2.749 & -.242 & & & \\
\hline & Gender & & 4.995 & 6.200 & .137 & & & \\
\hline & Income & 39 & .194 & .818 & .039 & & & \\
\hline \multirow[t]{2}{*}{ Step 2} & & & & & & .127 & .011 & .527 \\
\hline & Mindfulness & & -32.080 & 50.160 & -.117 & & & \\
\hline
\end{tabular}




\section{Hypothesis E: Mindfulness will be associated with lower cancer-specific}

distress (Figure 2, Arrow E). The hypothesis that higher mindfulness would be related to lower cancer-specific distress was tested by performing bivariate correlations followed by hierarchical multiple regressions controlling for relevant variables. In all analyses, control variables entered in the first step did not add significant explanatory power to the model. Bivariate correlations revealed higher mindfulness was negatively associated with cancer-specific distress $(\mathrm{r}=-.469, \mathrm{p}<.01$; see Table 4 for bivariate correlations $)$. This result maintained significance even after entering four control variables. Control variables, included age at diagnosis, stage of cancer, gender and income, were entered in the first block and mindfulness was entered in the second block. A multiple regression was preformed utilizing cancer-specific distress as the dependent variable and relevant control variables and mindfulness as predictors in order to determine if cancer-specific distress scores could be predicted as a function of mindfulness.

The overall model was statistically significant $\left(\Delta \mathrm{R}^{2}=.296 ; \mathrm{F}(5,36)=4.315, \mathrm{p}=\right.$ $.0002)$. Mindfulness was a significant individual predictor $(\beta=-.612, \mathrm{p}=.0002$; see Table 10). As indexed by the squared partial correlation coefficient, mindfulness accounted for $32.1 \%$ of the total variability in cancer-specific distress, indicating a large effect. The addition of mindfulness added significant explanatory power to the model and predicted lower cancer-specific distress. 


\section{Table 10}

$\underline{\text { Summary of hierarchical regression analyses entering mindfulness as the predictor of }}$ cancer-specific distress (IES-R, $\mathrm{N}=39$ )

\begin{tabular}{|c|c|c|c|c|c|c|c|c|}
\hline \multicolumn{2}{|c|}{ Variable } & $\mathrm{N}$ & B & SE B & $\beta$ & $\mathrm{R}^{2}$ & $\Delta \mathrm{R}^{2}$ & $\begin{array}{l}\mathrm{p} \text { of } \\
\Delta \mathrm{R}^{2}\end{array}$ \\
\hline \multirow[t]{5}{*}{ Step 1} & & & & & & .079 & .079 & .540 \\
\hline & Age at Diagnosis & & -.012 & .008 & -.233 & & & \\
\hline & Stage & & .038 & .079 & .081 & & & \\
\hline & Gender & & .032 & .183 & .030 & & & \\
\hline & Income & 39 & .012 & .024 & .084 & & & \\
\hline \multirow[t]{2}{*}{ Step 2} & & & & & & .375 & .296 & .0002 \\
\hline & Cancer-Specific Distress & & -4.915 & 1.190 & $-.612 * *$ & & & \\
\hline
\end{tabular}

$* \mathrm{p}<.05, * * \mathrm{p}<.01$

\section{Hypothesis F: Mindfulness will be associated with higher HRQOL (Figure 2,}

Arrow F). The hypothesis that higher mindfulness would be related to higher HRQOL was tested by performing bivariate correlations followed by hierarchical multiple regressions controlling for relevant variables. In all analyses, control variables entered in the first step, with the exception of age at diagnosis, did not add significant explanatory power to the model. This significance dropped out after the addition of mindfulness in step two. Bivariate correlations revealed higher mindfulness was positively associated with HRQOL ( $\mathrm{r}=.631, \mathrm{p}<.01$; see Table 4 for bivariate correlations). This result maintained significance even after entering four control variables. Control variables, included age at diagnosis, stage of cancer, gender and income, were entered in the first block and mindfulness was entered in the second block. A multiple regression was preformed utilizing HRQOL as the dependent variable and relevant control variables and mindfulness as predictors in order to determine if HRQOL scores could be predicted as a function of mindfulness. 
The overall model was statistically significant $\left(\Delta \mathrm{R}^{2}=.305 ; \mathrm{F}(5,36)=5.228, \mathrm{p}=\right.$ $.0001)$. Mindfulness was a significant individual predictor $(\beta=.634, \mathrm{p}=.0001$; see Table 11). As indexed by the squared partial correlation coefficient, traumatic distress appraisal accounted for $34.5 \%$ of the total variability in HRQOL, indicating a large effect. The addition of mindfulness added significant explanatory power to the model and predicted higher HRQOL.

\section{Table 11}

$\underline{\text { Summary of hierarchical regression analyses entering mindfulness as the predictor of }}$ $\underline{\text { HRQOL (FACT-L, N = 41) }}$

\begin{tabular}{|c|c|c|c|c|c|c|c|c|}
\hline \multicolumn{2}{|c|}{ Variable } & $\mathrm{N}$ & B & SE B & $\beta$ & $\mathrm{R}^{2}$ & $\Delta \mathrm{R}^{2}$ & $\begin{array}{l}\mathrm{p} \text { of } \\
\Delta \mathrm{R}^{2}\end{array}$ \\
\hline \multirow[t]{5}{*}{ Step 1} & & & & & & .116 & .116 & .321 \\
\hline & Age at Diagnosis & & .766 & .358 & $.340^{*}$ & & & \\
\hline & Stage & & 1.641 & 3.332 & .082 & & & \\
\hline & Gender & & 3.802 & 7.736 & .082 & & & \\
\hline & Income & 41 & .652 & 1.004 & .105 & & & \\
\hline \multirow[t]{2}{*}{ Step 2} & & & & & & .421 & .305 & .0001 \\
\hline & Mindfulness & & 219.959 & 50.566 & $.634 * *$ & & & \\
\hline
\end{tabular}

$$
* \mathrm{p}<.05, * * \mathrm{p}<.01
$$




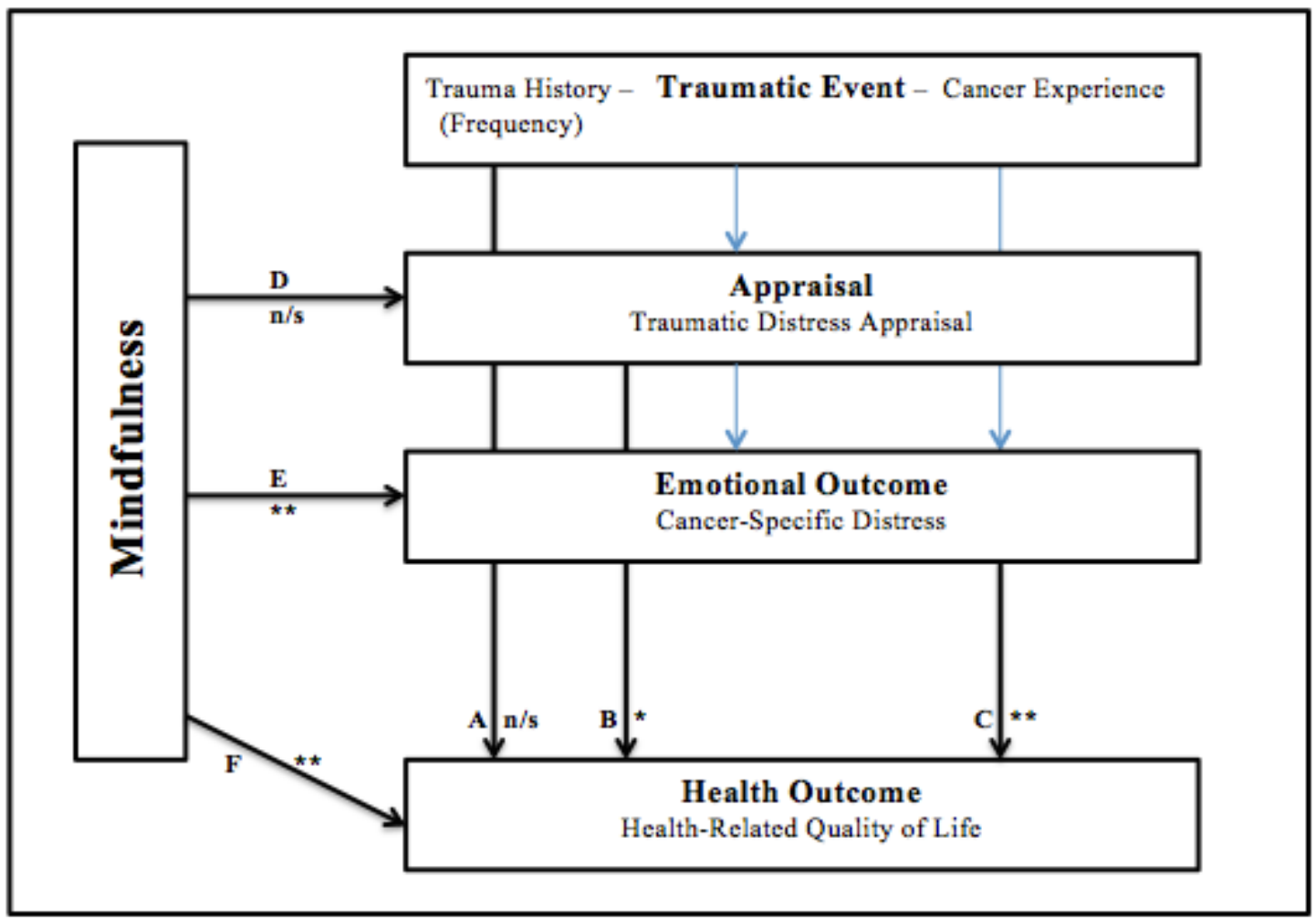

Figure 6: Summary of primary analyses for Aims 1 and $2 .{ }^{*} \mathrm{p}<.05,{ }^{*} \mathrm{p}<.01, \mathrm{n} / \mathrm{s}$ not significant

\section{Hypothesis G Mindfulness will moderate the effects of traumatic distress}

appraisal on HRQOL (Figure 3, Arrow G). To explore mindfulness as a potential moderator of the relationship between traumatic distress appraisal and HRQOL, assumptions of moderation were tested. The first assumption tested was to check if mindfulness was significantly correlated with either traumatic distress appraisal or HRQOL. Bivariate Pearson correlations were examined (see Table 4) and found that traumatic distress appraisal was not significantly correlated with mindfulness. HRQOL, the dependent variable was significantly correlated with mindfulness $(p<.01)$ violating the assumption that the potential moderator not be significantly associated with either the individual predictor (Kraemer et al., 2001) or dependent variable (Baron \& Kenny, 1986). 
As the assumption of collinearity has been violated, the hypothesis of mindfulness serving as moderator is unable to be empirically tested at this time. However, in order to explore the data, and to inform future work, moderation was tested in an exploratory fashion in order to generate hypotheses for future research, and to fulfill the plan of analyses in this dissertation. To explore mindfulness as a potential moderator of the relationship between traumatic distress appraisal and HRQOL, centered variables were created. In this hierarchical regression model, age at diagnosis, cancer stage, gender and income were added as covariates on the first step. Mean-centered mindfulness and meancentered traumatic distress appraisal were entered in step two. The overall model was statistically significant $\left(\Delta \mathrm{R}^{2}=.404 ; \mathrm{F}(6,33)=5.856, \mathrm{p}=.00005\right)$. Centered traumatic distress appraisal $(\beta=-.337, p=.014)$ and centered mindfulness $(\beta=.590, p=.0002$; see Table 12) were significant individual predictors.

Using the mean-centered mindfulness and mean-centered traumatic distress appraisal scores, a cross-product of these variables was calculated and entered on the second step of the regression equation. The overall model remained significant $\left(\Delta R^{2}=\right.$ $.405 ; \mathrm{F}(7,32)=4.898, \mathrm{p}=.0002)$, however, the cross-product was not significant $(\beta=$ $.046, \mathrm{p}=.747$; see Table 12). Both mean-centered mindfulness and mean-centered traumatic distress appraisal remained significant individual predictors with the addition of the cross-product within the model. As such, mindfulness does not appear to moderate the relationship between traumatic distress appraisal and HRQOL. 


\section{Table 12}

$\underline{\text { Summary of hierarchical regression analyses testing for moderation of mindfulness on }}$ $\underline{\text { traumatic distress appraisal and HRQOL (FACT-L, } \mathrm{N}=39 \text { ) }}$

\begin{tabular}{|c|c|c|c|c|c|c|c|c|}
\hline \multicolumn{2}{|c|}{ Variable } & $\mathrm{N}$ & B & SE B & $\beta$ & $\overline{\mathrm{R}^{2}}$ & $\overline{\Delta \mathrm{R}^{2}}$ & $\begin{array}{l}\mathrm{p} \text { of } \\
\Delta \mathrm{R}^{2}\end{array}$ \\
\hline \multirow[t]{5}{*}{$\overline{\text { Step } 1}$} & & & & & & .112 & .112 & .370 \\
\hline & Age at Diagnosis & & .766 & .370 & $.339 *$ & & & \\
\hline & Stage & & 1.164 & 3.517 & .056 & & & \\
\hline & Gender & & 3.831 & 7.933 & .082 & & & \\
\hline & Income & 39 & .631 & 1.046 & .100 & & & \\
\hline \multirow[t]{5}{*}{ Step 2} & & & & & & .516 & .404 & $\begin{array}{l}.0000 \\
5\end{array}$ \\
\hline & Centered Traumatic & & -.430 & .165 & $-.337^{*}$ & & & \\
\hline & Distress Appraisal & & & & & & & \\
\hline & Centered Mindfulness & & 206.64 & 48.69 & & & & \\
\hline & & & 3 & 2 & $.590 * *$ & & & \\
\hline \multirow[t]{9}{*}{ Step 2} & & & & & & .517 & .405 & .0002 \\
\hline & Centered Traumatic & & -.455 & .184 & $-.356^{*}$ & & & \\
\hline & Distress Appraisal & & & & & & & \\
\hline & Centered Mindfulness & & 207.62 & 49.45 & & & & \\
\hline & & & 3 & 7 & $.593 * *$ & & & \\
\hline & Cross-Product of & & -.968 & 2.974 & -.046 & & & \\
\hline & Centered Traumatic & & & & & & & \\
\hline & Distress Appraisal and & & & & & & & \\
\hline & Centered Mindfulness & & & & & & & \\
\hline
\end{tabular}

\section{Hypothesis H: Mindfulness will moderate the effects of cancer-specific}

distress on HRQOL (Figure 4, Arrow H). To evaluate mindfulness as a potential moderator of the relationship between cancer-specific distress and HRQOL, assumptions of moderation were tested. The first assumption tested was to check if mindfulness was significantly correlated with either cancer specific distress or HRQOL. Bivariate Pearson correlations were examined (see Table 4) and found that cancer-specific distress and HRQOL were significantly correlated with mindfulness (both $p<.01$ ) violating the 
assumption that the potential moderator not be significantly associated with either the individual predictor (Kraemer et al., 2001) or dependent variable (Baron \& Kenny, 1986).

As the assumption of collinearity has been violated, the hypothesis of mindfulness serving as moderator is unable to be empirically tested at this time. However, in order to explore the data and to inform future work, moderation was tested in an exploratory fashion in order to generate hypotheses for future research, and to fulfill the plan of analyses in this dissertation. To explore mindfulness as a potential moderator of the relationship between cancer-specific distress and HRQOL, centered variables were created. In this hierarchical regression model, age at diagnosis, cancer stage, gender and income were added as covariates on the first step. Mean-centered cancer-specific distress and mean-centered mindfulness were entered in step two. The overall model was statistically significant $\left(\Delta \mathrm{R}^{2}=.365 ; \mathrm{F}(6,34)=5.631, \mathrm{p}=.00009\right)$. Centered cancerspecific distress $(\beta=-.376, p=.018)$ and centered mindfulness $(\beta=.380, p=.029$; see Table 13) were significant individual predictors.

Using the mean-centered cancer-specific distress and mean-centered mindfulness, a cross-product of these variables was calculated and entered on the second step of the regression equation. The overall model remained significant $\left(\Delta \mathrm{R}^{2}=.365 ; \mathrm{F}(7,33)=\right.$ $4.701, \mathrm{p}=.0004)$, however, the cross-product was not significant $(\beta=-.032, \mathrm{p}=.816$; see Table 13). Both mean-centered cancer-specific distress and mean-centered mindfulness remained significant individual predictors with the addition of the cross-product within the model. As such, mindfulness does not appear to moderate the relationship between cancer-specific distress and HRQOL. 


\section{Table 13}

$\underline{\text { Summary of hierarchical regression analyses testing for moderation of mindfulness on }}$ cancer-specific distress and HRQOL (FACT-L, $\mathrm{N}=40$ )

\begin{tabular}{|c|c|c|c|c|c|c|c|c|}
\hline Variabl & & $\mathrm{N}$ & B & SE B & $\beta$ & $\mathrm{R}^{2}$ & $\Delta \mathrm{R}^{2}$ & $\begin{array}{l}\mathrm{p} \text { of } \\
\Delta \mathrm{R}^{2}\end{array}$ \\
\hline \multirow[t]{5}{*}{ Step 1} & & & & & & .134 & .134 & .256 \\
\hline & Age at Diagnosis & & .359 & .291 & .163 & & & \\
\hline & Stage & & -1.561 & 2.987 & -.079 & & & \\
\hline & Gender & & -.459 & 6.393 & -.010 & & & \\
\hline & Income & 40 & .1 .059 & .773 & .175 & & & \\
\hline \multirow[t]{3}{*}{ Step 2} & & & & & & .498 & .365 & .00009 \\
\hline & $\begin{array}{l}\text { Centered Cancer- } \\
\text { Specific Distress }\end{array}$ & & -16.035 & 6.446 & $-.376^{*}$ & & & \\
\hline & Centered Mindfulness & & 130.712 & 57.402 & $.380 *$ & & & \\
\hline \multirow{7}{*}{ Step 2} & & & & & & .499 & .365 & .0004 \\
\hline & $\begin{array}{l}\text { Centered Cancer- } \\
\text { Specific Distress }\end{array}$ & & -15.932 & 6.552 & $-.374 *$ & & & \\
\hline & Centered Mindfulness & & 127.743 & 59.576 & $.372 *$ & & & \\
\hline & Cross-Product of & & -20.361 & 86.786 & -.032 & & & \\
\hline & Centered Cancer- & & & & & & & \\
\hline & Specific Distress and & & & & & & & \\
\hline & Centered Mindfulness & & & & & & & \\
\hline
\end{tabular}

\section{Secondary Analyses}

\section{Secondary Analyses for Aim 1: Examine the relationship between trauma}

and HRQOL. In order to explore which potential subscales may account for the variance and significance in the primary analyses, correlations and hierarchical regressions were explored in detail. To explore if the overall total scores of predictor variables corresponded with subscales of HRQOL, bivariate Pearson correlations between traumatic distress appraisal, cancer-specific distress and the subscales of HRQOL were calculated (Table 14). 


\section{Table 14}

Bivariate Pearson correlations between trauma variables and subscales of the HRQOL

$\underline{(\text { FACT-L; } \mathrm{N}=46)}$

\begin{tabular}{lccccc}
\hline \multicolumn{1}{c}{ Variable } & $\begin{array}{c}\text { Physical } \\
\text { Well- } \\
\text { Being }\end{array}$ & $\begin{array}{c}\text { Social and } \\
\text { Family } \\
\text { Well- } \\
\text { Being }\end{array}$ & $\begin{array}{c}\text { Emotional } \\
\text { Well-Being }\end{array}$ & $\begin{array}{c}\text { Functional } \\
\text { Well-Being }\end{array}$ & $\begin{array}{c}\text { Lung } \\
\text { Cancer } \\
\text { Wellness }\end{array}$ \\
\hline $\begin{array}{l}\text { Traumatic } \\
\text { Distress Appraisal } \\
\begin{array}{l}\text { Cancer-Specific } \\
\text { Distress }\end{array}\end{array}$ & $\mathbf{- . 2 4 9}$ & $-.373 *$ & -.286 & -.262 & $-.334 *$ \\
\hline & $\mathbf{- . 5 9 2 * *}$ & $\mathbf{- . 1 2 5}$ & $\mathbf{- . 7 1 2 * *}$ & $\mathbf{- . 5 3 5 * *}$ & $\mathbf{- . 3 4 9 *}$ \\
\hline
\end{tabular}

$* \mathrm{p}<.05, * * \mathrm{p}<.01$ Note that correlations in bold are Spearman correlations

Hypothesis B: Examine the relationship between traumatic distress appraisal

and specific cancer outcomes. In order to test if traumatic distress appraisal predicted decrements in specific cancer outcomes, five hierarchical linear regressions were performed. In all analyses, control variables entered in the first step did not add significant explanatory power to the model. Bivariate correlations between traumatic distress appraisal and subscales of HRQOL demonstrated that traumatic distress appraisal was significantly correlated with social and family well-being $(r=-.373, p<.05)$, and lung cancer wellness $(\mathrm{r}=-.334, \mathrm{p}<.05$; see Table 14). Control variables, included age at diagnosis, stage of cancer, gender and income, were entered in the first block and traumatic distress appraisal was entered in the second block on hierarchical regressions using HRQOL subscales as the dependent variables.

Interestingly, traumatic distress appraisal was significantly associated with social/family well-being and emotional well-being (see Figure 7). As indexed by the squared partial correlation coefficient, traumatic distress appraisal accounted for $13.4 \%$ of the total variability in the social and family well-being subscale, accounted for $12.5 \%$ 
of the total variability in the emotional well-being subscale, both medium effects. The addition of traumatic distress appraisal added significant explanatory power to both models and predicted lower social and family well-being, and lower emotional wellbeing. Traumatic distress appraisal was not related to the other three subscales.

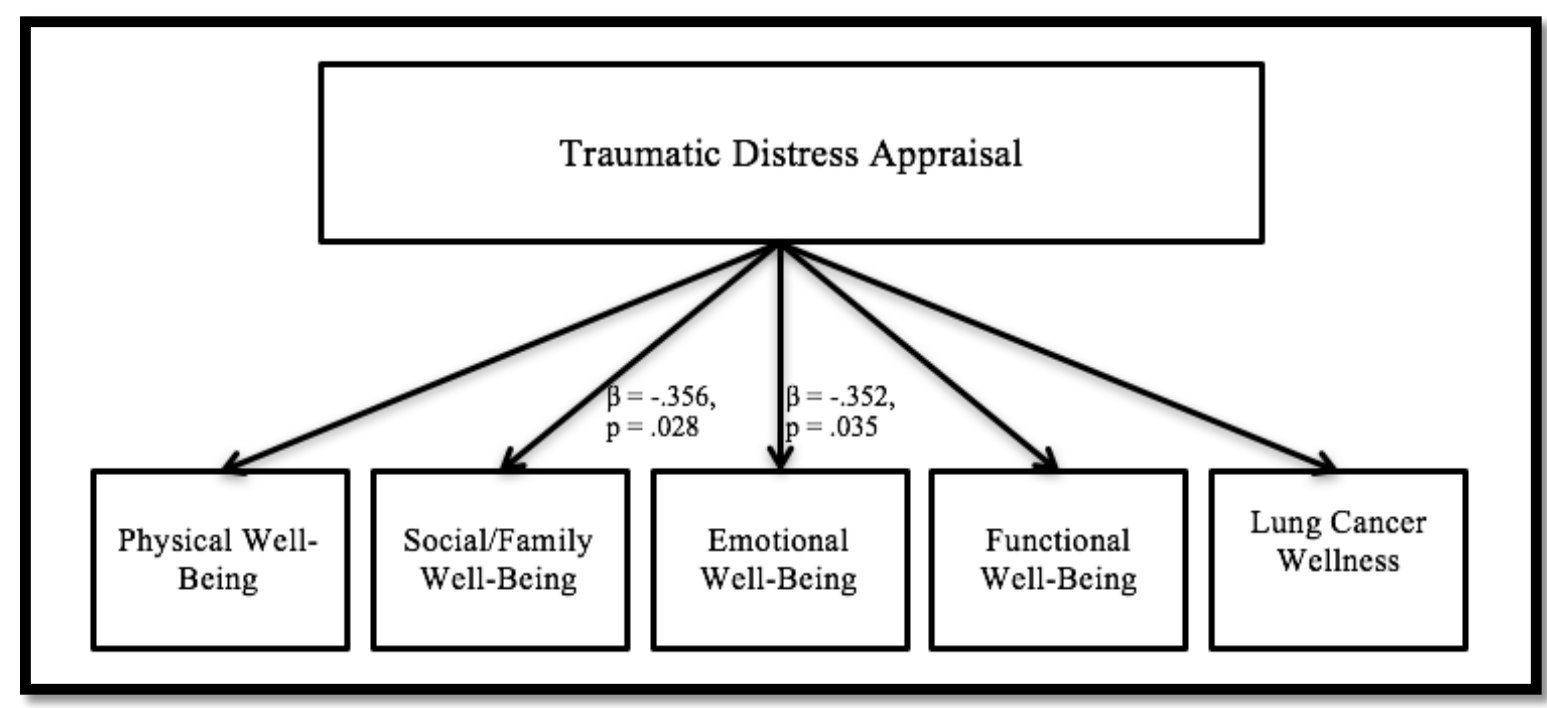

Figure 7. Associations between Traumatic Distress Appraisal and subscales of HRQOL.

\section{Hypothesis C: Examine the relationship between cancer-specific distress and}

HRQOL. In order to test if cancer-specific distress predicted decrements in specific cancer outcomes, five hierarchical linear regressions were performed. In all analyses, control variables entered in the first step that added significant explanatory power to the model, became not significant with the addition of the predictor variable with the exception of age at diagnosis for the social and family well-being subscale. Bivariate Spearman correlations between cancer-specific distress and subscales of HRQOL demonstrated that cancer-specific distress was significantly correlated with physical wellbeing $(r=-.592, p<.01)$, emotional well-being $(r=-.712, p<.01)$, functional well-being $(\mathrm{r}=-.535, \mathrm{p}<.01)$, and lung cancer wellness $(\mathrm{r}=-.349, \mathrm{p}<.05$; see Table 14). Cancerspecific distress was not correlated with social and family well-being. 
Control variables including age at diagnosis, cancer stage, gender and income were entered in the first block, cancer-specific distress was entered in the second block. Each subscale of the FACT-L (physical well-being, social/family well-being, emotional well-being, functional well-being and lung cancer wellness) was entered as dependent variables for a total of five regressions. Cancer-specific distress was significantly associated with physical well-being, emotional well-being, functional well-being, and lung cancer wellness (see Figure 8). As indexed by the squared partial correlation coefficient, cancer-specific distress accounted for $30.8 \%$ of the total variability in the physical well-being subscale, $30.4 \%$ of the total variability in the emotional well-being subscale, $32.1 \%$ of the total variability in the functional subscale, all considered large effects. Cancer-specific distress accounted for $15.3 \%$ of the total variability in the lung cancer wellness subscale, indicating a medium effect. The addition of cancer-specific distress added significant explanatory power to all models and predicted lower well-being in all four domains (physical, emotional, functional and lung cancer wellness). Cancerspecific distress was not related to social and family well-being

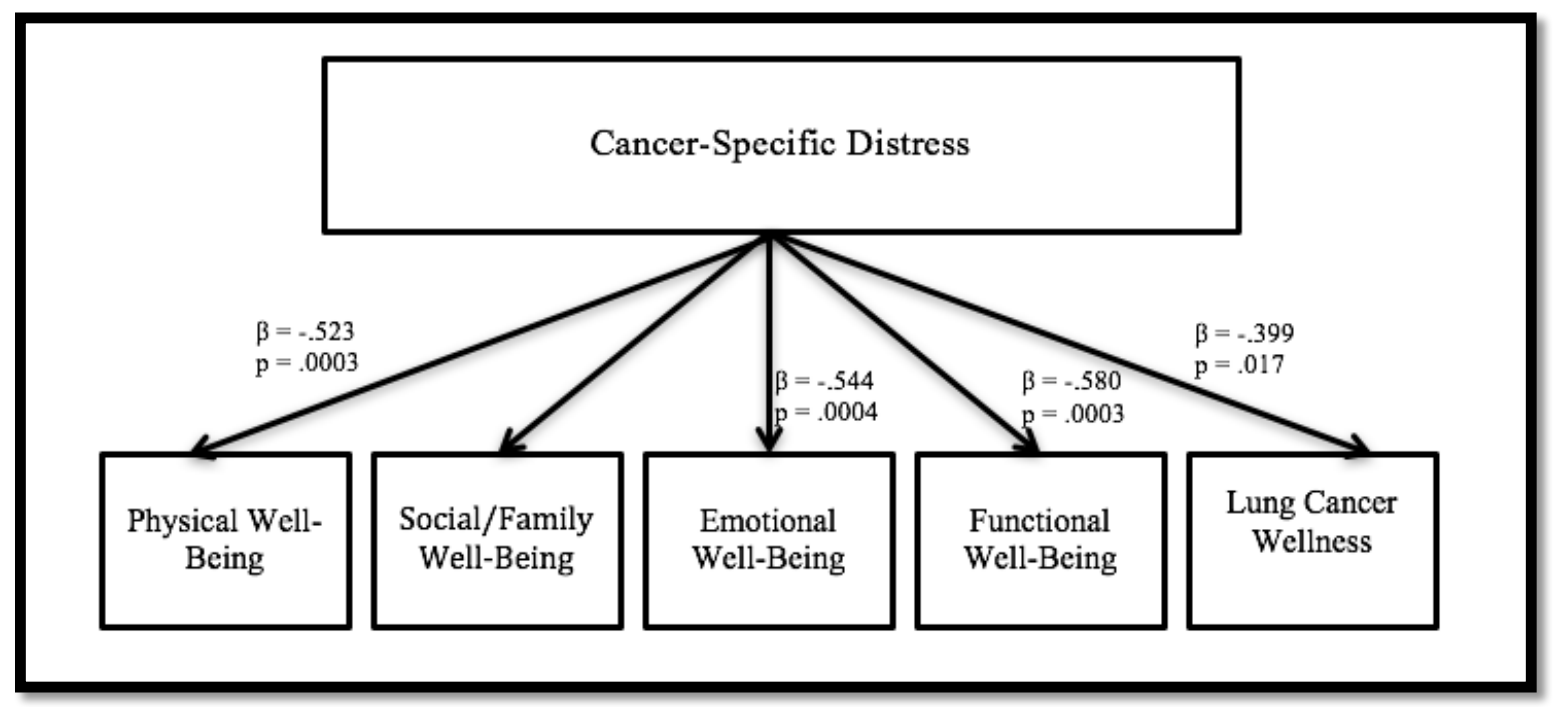

Figure 8. Associations between Cancer-Specific Distress and subscales of HRQOL. 
In order to test if subscales of cancer-specific distress predicted decrements in HRQOL, three hierarchical linear regressions were performed. Bivariate Pearson correlations between cancer-specific distress subscales (intrusions, hyperarousal, and avoidance) and HRQOL demonstrated that all three subscales (intrusion: $\mathrm{r}=-.502, \mathrm{p}<$ .01 ; hyperarousal $\mathrm{r}=-.526, \mathrm{p}<.01$, avoidance $\mathrm{r}=-.533, \mathrm{p}<.01$ ) were significantly correlated with HRQOL (see Table 15).

\section{Table 15}

Bivariate Pearson correlations between the subscales of the cancer-specific distress scale and HRQOL (FACT-L; $\mathrm{N}=46$ )

\begin{tabular}{lccc}
\hline \multicolumn{1}{c}{ Variable } & Intrusions & Hyperarousal & Avoidance \\
\hline $\begin{array}{l}\text { Health-Related Quality of } \\
\text { Life }\end{array}$ & $-.502 * *$ & $-.526^{* *}$ & $-.533^{* *}$ \\
\hline${ }^{*} \mathrm{p}<.05, * * \mathrm{p}<.01$ & & &
\end{tabular}

Control variables including age at diagnosis, cancer stage, gender and income were entered in the first block, each subscale (intrusions, hyperarousal and avoidance) was separately entered in the second block, and total HRQOL was entered as the dependent variable for a total of three regressions. All three subscales were significantly associated with HRQOL (see Figure 9). As indexed by the squared partial correlation coefficient, intrusions accounted for $23.2 \%$ of the total variability in HRQOL, hyperarousal accounted for $23.5 \%$ of the total variability in HRQOL, and voidance accounted for $27.0 \%$ of the total variability in HRQOL, all considered large effects. The addition of each subscale added significant explanatory power to all models and predicted lower 


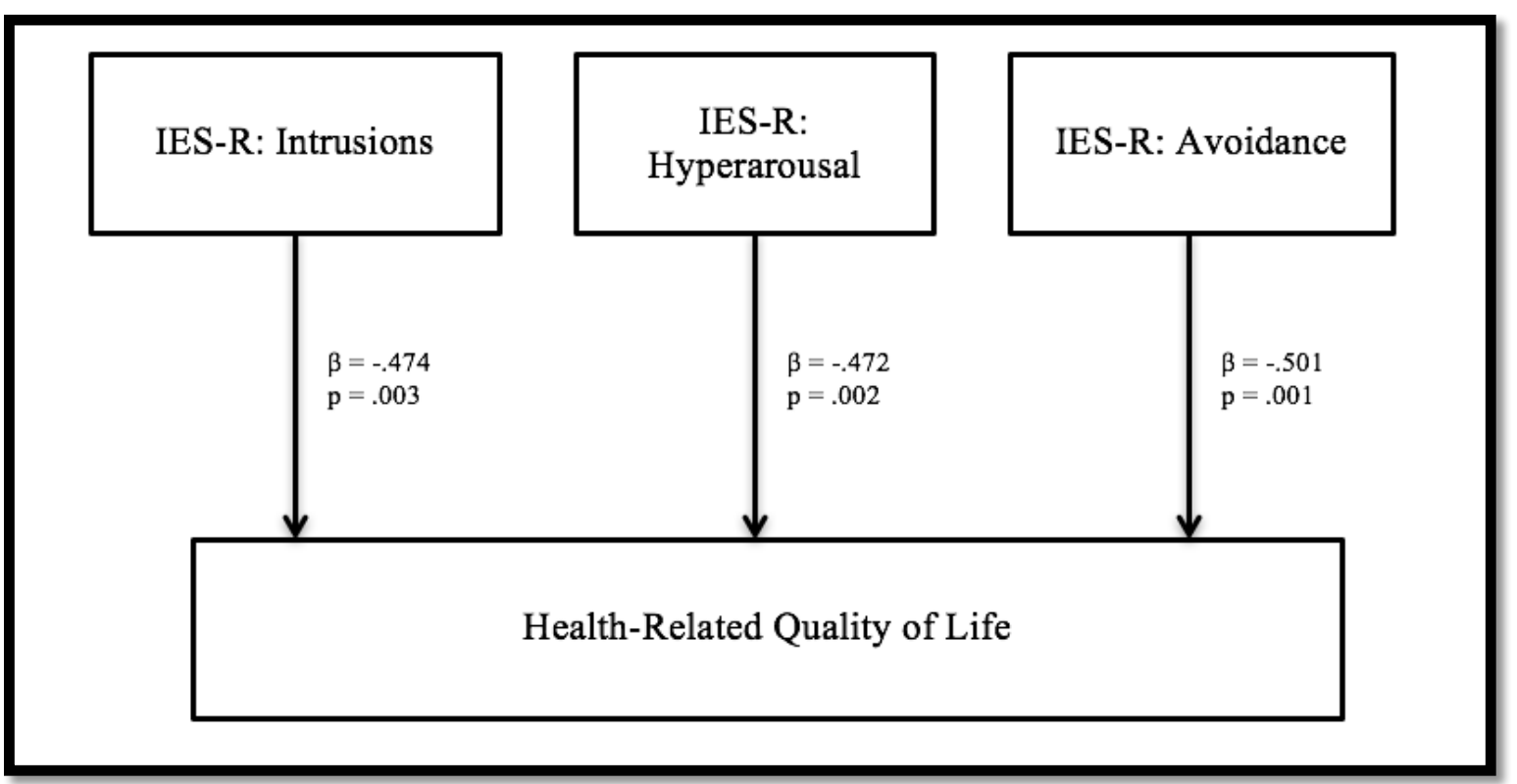

Figure 9. Associations between subscales of cancer-specific distress and total HRQOL.

\section{Hypothesis E: Examine the relationship between mindfulness and cancer-}

specific distress. In order to test if mindfulness predicted improvements in cancerspecific distress subscales, three hierarchical linear regressions were performed. Bivariate Spearman correlations between mindfulness and subscales of cancer-specific distress (Table 16) demonstrated that mindfulness was significantly correlated with all three subscales (intrusion: $\mathrm{r}=-.375, \mathrm{p}<.05$; hyperarousal $\mathrm{r}=-.466, \mathrm{p}<.01$, avoidance $\mathrm{r}=$ $.519, \mathrm{p}<.01)$

\section{Table 16}

$\underline{\text { Bivariate Spearman correlations between the subscales of the cancer-specific distress }}$ $\underline{\text { scale and mindfulness (IES-R; } \mathrm{N}=46 \text { ) }}$

\begin{tabular}{lccc}
\hline \multicolumn{1}{c}{ Variable } & Intrusions & Hyperarousal & Avoidance \\
\hline Mindfulness & $-.375^{*}$ & $-.466 * *$ & $-.519 * *$ \\
\hline$* \mathrm{p}<.05, * * \mathrm{p}<.01$ & & &
\end{tabular}

Control variables including age at diagnosis, cancer stage, gender and income were entered in the first block, mindfulness was separately entered in the second block, 
and each subscale (intrusions, hyperarousal and avoidance) was entered as the dependent variable for a total of three regressions. Mindfulness was significantly associated with all three subscales (see Figure 10). As indexed by the squared partial correlation coefficient, mindfulness accounted for $16.6 \%$ of the total variability in the intrusions subscale, indicating a medium effect. Mindfulness accounted for $22.4 \%$ of the total variability in the hyperarousal subscale, and $38.4 \%$ of the total variability in the avoidance subscale, both considered large effects. The addition of mindfulness added significant explanatory power to all models and predicted lower cancer-specific distress in all three domains (intrusions, hyperarousal and avoidance).

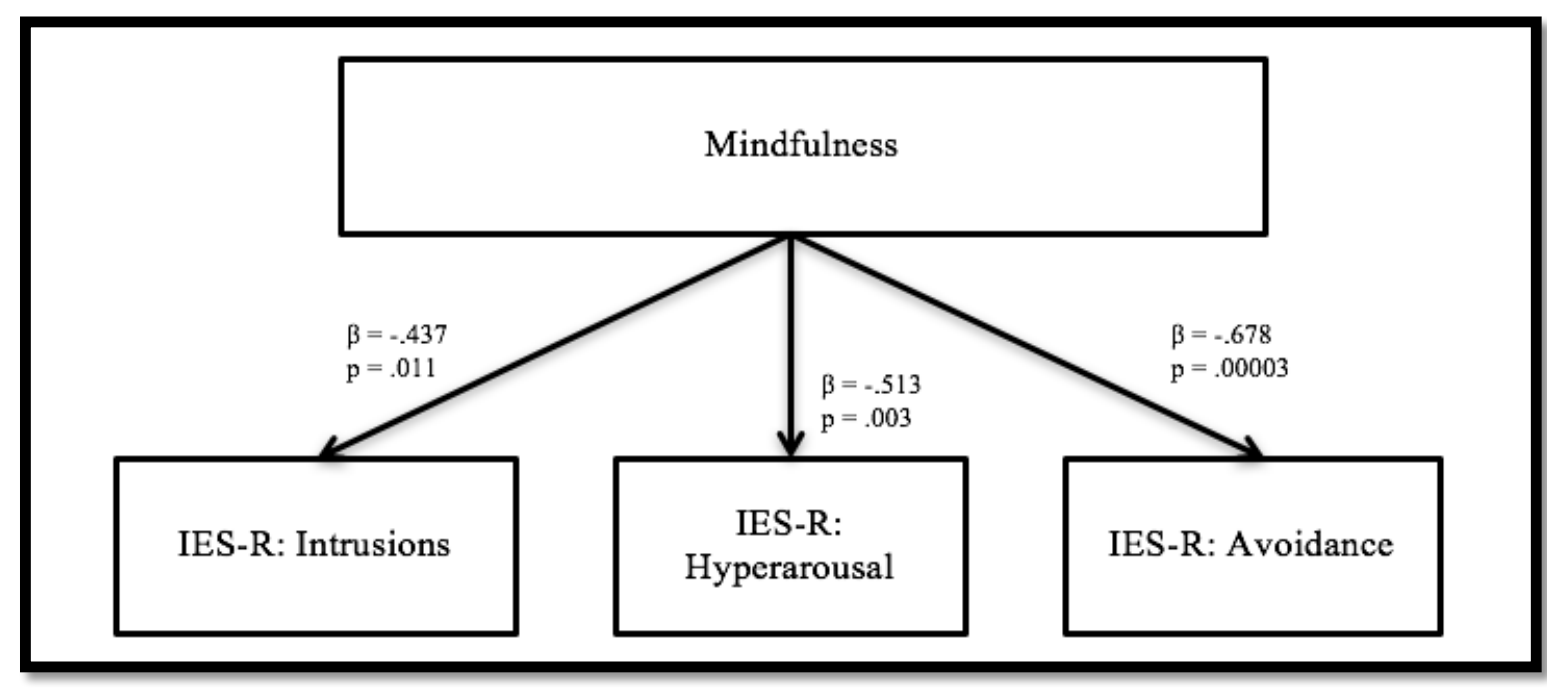

Figure 10. Associations between total mindfulness and cancer-specific distress subscales.

In order to test if subscales of mindfulness predicted improvements in cancerspecific distress, five hierarchical linear regressions were performed. Bivariate Spearman correlations between mindfulness subscales (observe, describe, act with awareness, nonjudgment, nonreactivity) and cancer-specific distress demonstrated that act with awareness $(\mathrm{r}=-.614, \mathrm{p}<.01)$ and nonjudgment $(\mathrm{r}=-.415, \mathrm{p}<.01$; see Table 17$)$ were significantly negatively correlated with cancer-specific distress. Control variables 
including age at diagnosis, cancer stage, gender and income were entered in the first block, each subscale (observe, describe, act with awareness, nonjudgment, nonreactivity) was separately entered in the second block, and total cancer-specific distress was entered as the dependent variable for a total of five regressions.

\section{Table 17}

Bivariate Spearman correlations between the subscales of mindfulness and cancerspecific distress total score (IES-R; $\mathrm{N}=46$ )

\begin{tabular}{lccccc}
\hline \multicolumn{1}{c}{ Variable } & Observe & Describe & $\begin{array}{c}\text { Act with } \\
\text { Awareness }\end{array}$ & $\begin{array}{c}\text { Non- } \\
\text { judgment }\end{array}$ & $\begin{array}{c}\text { Non- } \\
\text { reactivity }\end{array}$ \\
\hline $\begin{array}{l}\text { Cancer-Specific } \\
\text { Distress }\end{array}$ & .096 & -.280 & $-.614^{* *}$ & $-.415^{* *}$ & -.029 \\
\hline$* * \mathrm{p}<.01$ & & & & &
\end{tabular}

Three subscales were significantly associated with cancer-specific distress. Describe, act with awareness, and nonjudgment (see Figure 11) were significantly associated with improvements in cancer-specific distress. As indexed by the squared partial correlation coefficient, the describe subscale accounted for $18.2 \%$ of the total variability in cancer-specific distress, and nonjudgment accounted for $18.6 \%$ of the total variability in cancer-specific distress, both considered medium effects. The act with awareness subscale accounted for $43.2 \%$ of the total variability in the cancer-specific distress, which is considered a large effect. The addition of the subscales (describe, act with awareness and nonjudgment) added significant explanatory power to these three models and predicted lower cancer-specific distress. 


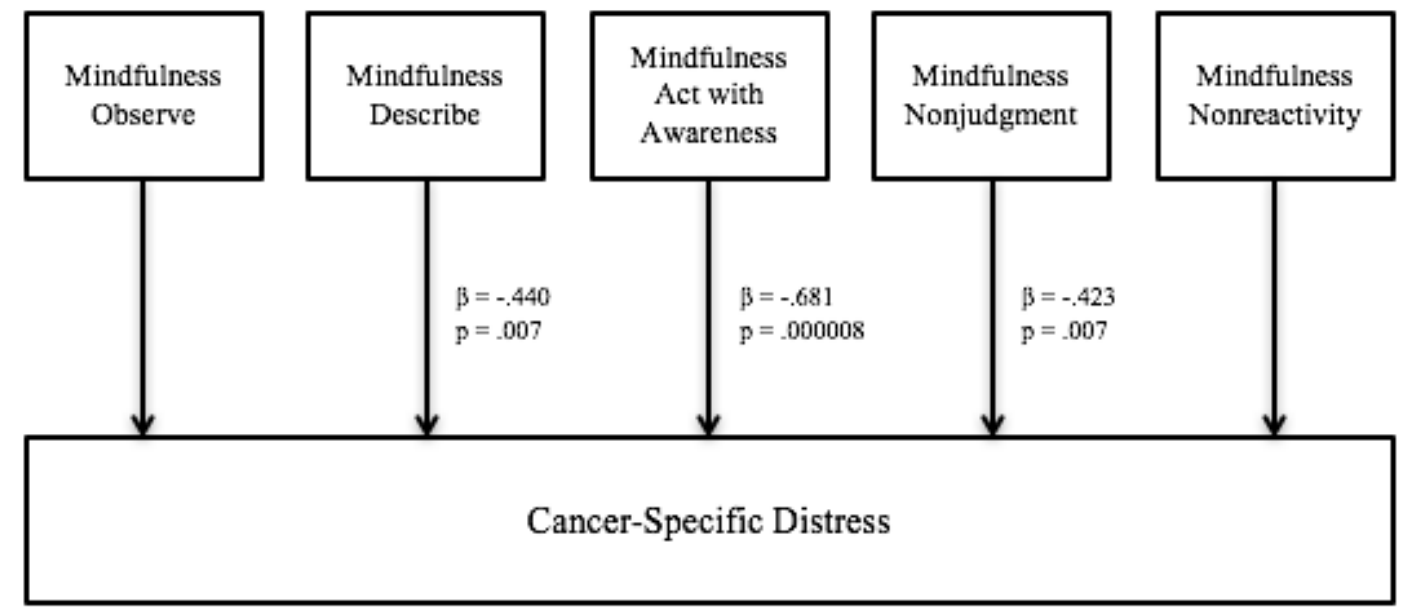

Figure 11. Associations between the subscales of mindfulness and total cancer-specific distress.

\section{Hypothesis F: Examine the relationship between mindfulness and HRQOL.}

In order to test if mindfulness predicted improvements in HRQOL subscales, five hierarchical linear regressions were performed. Bivariate Spearman correlations between mindfulness and subscales of HRQOL demonstrated that mindfulness was significantly correlated with all five subscales (physical well-being: $r=.566, \mathrm{p}<.01$; social/family well-being $\mathrm{r}=.379, \mathrm{p}<.01$, emotional well-being $=.481, \mathrm{p}<.05$; functional well-being $=.458, \mathrm{p}<.01$; lung cancer wellness $\mathrm{r}=.366, \mathrm{p}<.05$; Table 18).

\section{Table 18}

$\underline{\text { Bivariate Spearman correlations between mindfulness and specific cancer outcomes }}$

$\underline{\text { (FACT-L; } \mathrm{N}=46)}$

\begin{tabular}{cccccc}
\hline Variable & $\begin{array}{c}\text { Physical } \\
\text { Well- } \\
\text { Being }\end{array}$ & $\begin{array}{c}\text { Social and } \\
\text { Family } \\
\text { Well- } \\
\text { Being }\end{array}$ & $\begin{array}{c}\text { Emotional } \\
\text { Well-Being }\end{array}$ & $\begin{array}{c}\text { Functional } \\
\text { Well-Being }\end{array}$ & $\begin{array}{c}\text { Lung } \\
\text { Cancer } \\
\text { Wellness }\end{array}$ \\
\hline Mindfulness & $.566^{* *}$ & $.379^{* *}$ & $.481^{*}$ & $.548^{* *}$ & $.366^{*}$ \\
\hline
\end{tabular}

$* p<.05, * * p<.01$ 
Control variables including age at diagnosis, cancer stage, gender and income were entered in the first block, mindfulness was entered in the second block. Each subscale of the FACT-L (physical well-being, social/family well-being, emotional wellbeing, functional well-being and lung cancer wellness) was entered as dependent variables for a total of five regressions. Control variables that were significant in block one, became not significant with the addition of the predictor variable in the second block. Mindfulness was significantly associated with four subscales: physical well-being, emotional well-being, functional well-being, and lung cancer wellness (see Figure 12).

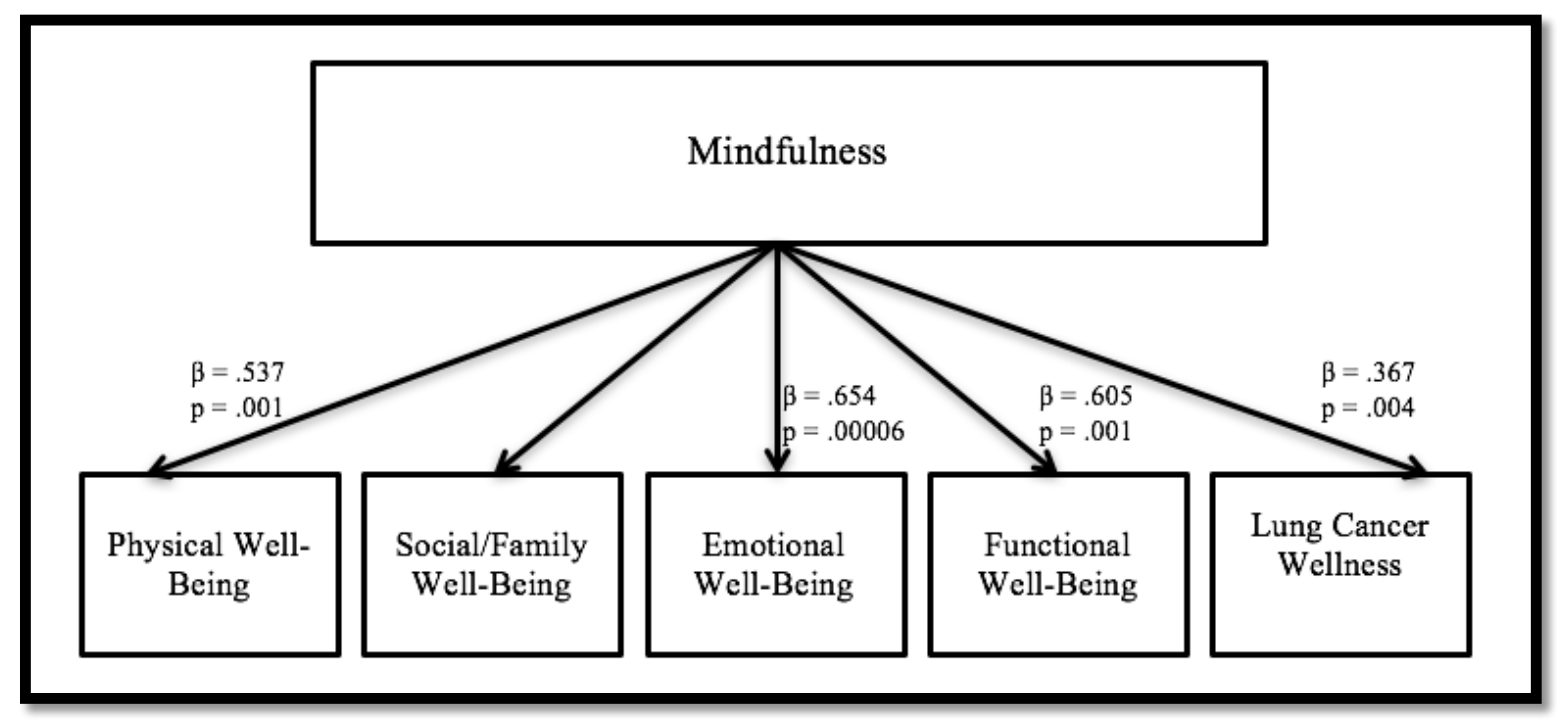

Figure 12. Associations between total mindfulness and subscales of HRQOL.

As indexed by the squared partial correlation coefficient, mindfulness accounted for $26.4 \%$ of the total variability in the physical well-being subscale, $36.2 \%$ of the total variability in the emotional well-being, $28.7 \%$ of the total variability in the functional well-being subscale, all considered large effects. Mindfulness accounted for $10.6 \%$ of the total variability of the lung cancer wellness subscale, which is considered a medium effect. The addition of mindfulness added significant explanatory power to all models and predicted higher HRQOL in all four domains (physical well-being, emotional well- 
being, functional well-being, and lung cancer wellness). Mindfulness was not associated with the social and family well-being subscale.

In order to test if the subscales of mindfulness predicted improvements in HRQOL, five hierarchical linear regressions were performed. Bivariate Spearman correlations between mindfulness subscales (observe, describe, act with awareness, nonjudgment, nonreactivity) and HRQOL demonstrated that describe $(\mathrm{r}=.548, \mathrm{p}<.01)$, act with awareness $(r=.529, \mathrm{p}<.01)$ and nonjudgment $(\mathrm{r}=.333, \mathrm{p}<.05$; see Table 19$)$ were significantly positively correlated with HRQOL.

\section{Table 19}

Bivariate Spearman correlations between the subscales of mindfulness and total HRQOL

$\underline{(F A C T-L ; ~} \mathrm{N}=46)$

\begin{tabular}{cccccc}
\hline Variable & Observe & Describe & $\begin{array}{c}\text { Act with } \\
\text { Awareness }\end{array}$ & $\begin{array}{c}\text { Non- } \\
\text { judgment }\end{array}$ & $\begin{array}{c}\text { Non- } \\
\text { reactivity }\end{array}$ \\
\hline $\begin{array}{l}\text { Health-Related } \\
\text { Quality of Life }\end{array}$ & .133 & $.548^{* *}$ & $.529 * *$ & $.333^{*}$ & .245 \\
\hline
\end{tabular}

$* \mathrm{p}<.05, * * \mathrm{p}<.01$

Control variables including age at diagnosis, cancer stage, gender and income were entered in the first block, each subscale (observe, describe, act with awareness, nonjudgment, nonreactivity) was separately entered in the second block, and total HRQOL was entered as the dependent variable for a total of five regressions. Two subscales, describe and act with awareness (see Figure 13), were significantly associated with improvements in HRQOL. 


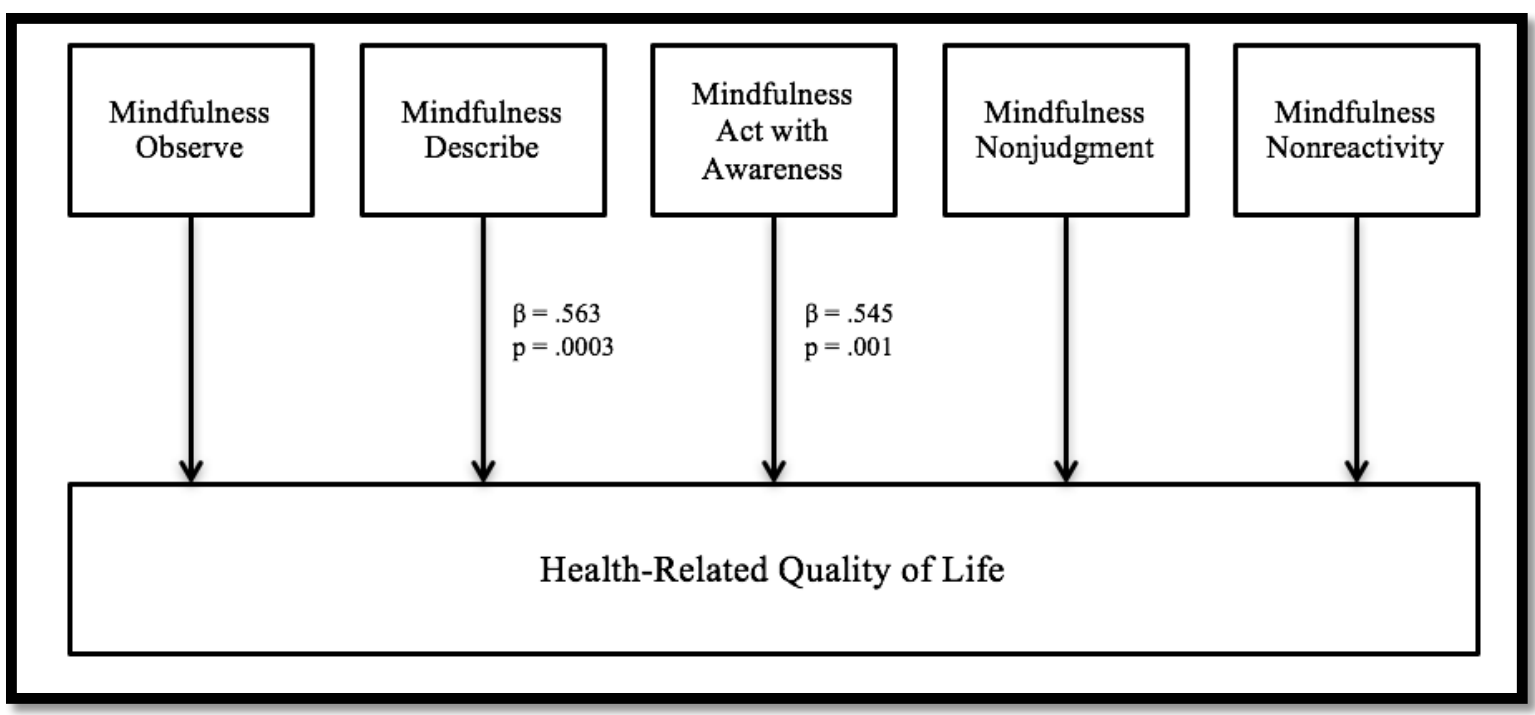

Figure 13. Associations between subscales of mindfulness and total HRQOL.

As indexed by the squared partial correlation coefficient, the describe subscale accounted for $30.7 \%$ of the total variability in HRQOL, and the act with awareness subscale accounted for $28.4 \%$ of the total variability in HRQOL, both considered large effects. The addition of the subscales (describe and act with awareness) added significant explanatory power to these two models and predicted higher HRQOL. 


\section{DISCUSSION}

\section{Summary}

This exploratory cross-sectional study investigated how three correlates of stress and coping - trauma, HRQOL and mindfulness may be related to one another in a sample of non-small cell lung cancer patients. The intention of the study was to examine how dispositional mindfulness, the innate ability to engage in non-judgmental present moment focus, was associated with both trauma and HRQOL. The relationships between trauma, HRQOL and mindfulness have been studied extensively in other populations, but had not yet been examined in lung cancer samples. This study employed three aims in order to extend demonstrated relationships within the literature to lung cancer patients: (1) it explored the relationship between trauma (as measured by frequency of traumatic events, traumatic distress appraisal and cancer-specific distress) and HRQOL (Hypotheses A, B and $\mathrm{C}$; Figure 2); (2) it examined the relationships of mindfulness with trauma and HRQOL (Hypotheses D, E and F; Figure 2); and (3) it explored the potential moderating impact of mindfulness on trauma and HRQOL.

As hypothesized, greater traumatic distress appraisal and cancer-specific distress were associated with lower HRQOL, but frequency of traumatic events was not associated with HRQOL. Additionally, mindfulness was significantly associated with decreased cancer-specific distress and higher HRQOL, but was unrelated to traumatic distress appraisal. Finally, exploratory analyses did not support the notion that 
mindfulness moderates the effects of trauma on HRQOL. Although the directionality of the observed relationships can not be determined from these cross-sectional data, the results do suggest the possibility that (1) trauma factors engender a vulnerability to having poorer HRQOL and (2) mindfulness may serve as a protective factor in psychological adjustment to lung cancer and can improve quality of life.

There are several important implications of the results. The majority of the sample (80.9\%) conceptualized their lung cancer experience as traumatic, and indicated that it was severely traumatic overall. A visual inspection of distress at the time of diagnosis showed that distress was high both at diagnosis and remained high throughout treatment. The results support the utility of screening for both lifetime traumatic events, and cancerspecific distress, prior to the start of treatment (at diagnosis) in order to assess vulnerability factors that may negatively impact HRQOL. Both traumatic distress appraisal and cancer-specific distress were negatively associated with HRQOL. Dispositional mindfulness was associated with higher HRQOL, despite the distress present in the sample. These relationships indicate that lung cancer patients may respond favorably to a mindfulness-based psychosocial intervention to improve HRQOL. Given that lung cancer patients are often diagnosed in later stages, selecting an intervention that may improve HRQOL at the end of life is an important treatment consideration. This study supports the clinical utility of piloting a mindfulness-based intervention for lung cancer patients, given the robust associations seen within this sample. The results are now discussed in detail.

\section{Main Findings}

\section{Aim 1: Examine the relationship between trauma and HRQOL}




\section{Hypothesis A: frequency of traumatic events will be associated with poorer}

HRQOL. The present study revealed that retrospective report of lifetime trauma exposure (frequency of traumatic events in one's history) was not related to HRQOL in the context of lung cancer. This is inconsistent with other findings that found strong relationships between trauma history and HRQOL (e.g., DuHamel et al., 2001; Green et al., 2000). There was one study, however, that found that frequency of traumatic events was not significantly predictive of HRQOL at baseline assessment (prior to the start of treatment) of recently diagnosed ovarian cancer patients (Lutgendorf et al., 2013). Researchers found that frequency of traumatic events in one's history only became significant at the one-year follow up after controlling for treatment variables such as having received chemotherapy (Lutgendorf et al., 2013); this could indicate that initial assessment of the impact of trauma history on HRQOL at baseline may be premature. It may also be the case that the timing of the traumatic event is more predictive of HRQOL, as compared to cumulative lifetime exposure. For example, Green and colleagues (2000) found that it was recent exposure to traumatic events, not remote events (i.e. childhood trauma), that predicted lower well-being and higher distress in a sample of breast cancer patients. The data for the timing of the traumatic events was not complete in this data collection (due to purposeful omission by the participants), preventing analysis of these relationships. It may be that the presence of trauma in one's history is less predictive of emotional dysregulation and distress than traumatic distress appraisal following a traumatic event.

\section{Hypothesis B: Traumatic distress appraisal will be associated with poorer}

HRQOL. Primary analyses revealed that patients with higher traumatic distress appraisal experienced lower HRQOL. This finding is consistent with other studies that have 
examined the relationship between distress related to one's trauma history and HRQOL in patients with breast cancer (Golden-Kreutz et al., 2005; Green et al., 2000), and a sample of heterogeneous cancer types (Jahn et al., 2012). This finding suggests that appraisal of lifetime traumatic events is an important predictor of current HRQOL in cancer patients. Overall, the current results support the notion that cancer patients experience considerable distress related to both lifetime traumatic events and their cancer diagnosis. The most common type of traumatic event reported was having a serious lifethreatening illness, with $80.9 \%$ of the sample reporting their lung cancer as a traumatic event and indicating that it was highly traumatic $(\mathrm{M}=6.07, \mathrm{SD}=1.13)$. This finding supports the utility of providing assessment and screening for lifetime stressors and perception of distress in cancer patients at the time of diagnosis (Golden-Kreutz et al., 2005).

This finding adds potentially adds validity to the theory that it is appraisal and not exposure to traumatic events that makes adjustment to subsequent stressors more difficult. Indeed, theoretical models have proposed that cognitive appraisals of trauma mediate the development of psychopathology and distress (Ehlers \& Clark, 2000). These appraisals include the assessment of thoughts, feelings, reactions and behaviors in response to a potentially traumatic event (DePrince, Chu, \& Pineda, 2011). There is considerable evidence in the literature that shows that it is negative appraisals of traumatic experiences that predict future distress, as opposed to the experience of the traumatic events themselves (e.g., Ellis, Nixon, \& Williamson, 2009; Martin, Cromer, DePrince, \& Freyd, 2013). In this regard, this study supports the theory that appraisal of traumatic events, and one's reactions to them, is more important in predicting decreased 
well-being than having experienced the events alone. It should be noted, however, that the data utilized to assess for traumatic distress appraisal was taken from a measure that has yet to be validated in the literature. Given the us of an invalidated measure, the results here should be interpreted with caution and should be replicated with a traumatic distress appraisal measure such as The Posttraumatic Cognitions Inventory, (PCTI; Foa, Ehlers, Clark, Tolin, \& Orsillo, 1999).

Secondary analyses showed that traumatic distress appraisal was only negatively associated with two subscales of HRQOL: social and family well-being and emotional well-being. Traumatic distress can often have considerable impact on interpersonal relationships, often causing the individual who experienced the potentially traumatic event to feel isolated and misunderstood (Dorahy, 2010; Wilson, Droždek, \& Turkovic, 2006). Elements of shame and guilt are often also present when traumatic distress is endorsed, causing further feelings of isolation and self-blame (Tangney, Miller, Flicker, \& Barlow, 1996). These processes might explain why this particular domain of HRQOL was negatively impacted. Traumatic distress appraisal has been shown to be associated with higher emotional dysregulation and lower HRQOL (Ellis, Nixon, \& Williamson, 2009), which could explain the decreased emotional well-being following traumatic distress appraisal of lifetime trauma exposure. The other three subscales of HRQOL (physical well-being, functional well-being, and lung cancer wellness) are all associated with disease-specific experiences. Traumatic distress appraisal is potentially a global assessment of lifetime trauma and would not be expected to be associated with these particular subscales due to their specific focus on the cancer experience. However, as noted, this measure has yet to be validated in the literature, so the construct being 
measured has yet to be proven. Both the emotional and social well-being subscales are most strongly linked to psychosocial experiences and could be most impacted by psychosocial interventions (Cella et al., 1995). The associations found within this analysis indicate that cancer patients that endorse high traumatic distress appraisal would potentially benefit most from psychosocial interventions, such as mindfulness-based therapy. Future research could also clarify factors that affect traumatic distress appraisal, thereby impacting HRQOL among patients with cancer. Such factors that could be explored further include coping styles and social support.

\section{Hypothesis C: Cancer-specific distress will be associated with poorer HRQOL.}

Primary analyses revealed that cancer-specific distress was negatively associated with HRQOL. This result is consistent with studies that have examined this relationship in other cancer samples (Gold et al., 2012; Hwang et al., 2003; Kang, Park, \& McArdle, 2012; Kangas et al., 2012; Shand, Cowlishaw, et al., 2014; Wachen et al., 2014). Interestingly, self-reported cancer-specific distress in this sample was lower than other samples studied (e.g., urogenital, lung, breast and other cancer types; Mystakidou et al., 2012). This may be because this sample's time since diagnosis ranged between one month to just under five years. This wide range of time since diagnosis, and treatment, may have allowed patients to adapt and cope with the initial stress burden of the cancer diagnosis, which could cause lower cancer-specific distress scores. Despite having lower cancer-specific distress as compared to other populations, however, results indicate that there is still a robust inverse relationship between cancer-specific distress and HRQOL. This result echoes previous findings that PTSS in cancer patients can have considerable 
impact on one's HRQOL and cancer experience (e.g., Andrykowski, Steffens, Bush, \& Tucker, 2015).

Secondary exploratory analyses of specific HRQOL sub-categories revealed that participants who reported higher cancer-specific distress had lower physical, emotional, functional and lung cancer-specific well-being, while no associations with social and family well-being were observed. The results are consistent with studies that reported relationships between higher cancer-specific distress and several domains of HRQOL (Lehto, Ojanen, \& Kellokumpu-Lehtinen, 2005; Ristvedt \& Trinkaus, 2009). In a study of patients with rectal cancer, it was found that men were more likely to have a significant association between cancer-specific distress and the social and family wellbeing subscale as compared to women (Ristevdt \& Trinkaus, 2009). As the current lung cancer sample was predominantly female, this may explain why this subscale was not impacted. It may be that women are more likely to utilize their social support networks, while men are fearful of becoming a burden on their families. Gender roles within the family unit may be impacting this finding. Future work with lung cancer patients should include a higher sampling of men to determine if men are more likely to endorse lower social and family well-being in relation to cancer-specific distress, as seen in the literature.

Secondary analyses also revealed that all three subscales of cancer-specific distress (intrusions, hyperarousal and avoidance) were negatively associated with overall HRQOL. In this respect, there was no single subscale that was driving the significance of the relationship between overall cancer-specific distress and HRQOL. Instead, it is clear that all three symptom subscales contribute to overall lower HRQOL. This finding is 
consistent with a study of gynecologic cancer patients that found that all three subscales were significantly related to overall HRQOL (Shand et al., 2014). This demonstrates that not only do cancer patients endorse all three types of PTSS (intrusions, hyperarousal and avoidance), but that all three are associated with HRQOL. Future work, with larger samples, should begin to explore if specific subscales of cancer-specific distress correspond with particular subscales of HRQOL to further understand these potentially unique relationships.

\section{Aim 2: Examine the relationships of mindfulness with trauma and HRQOL.}

\section{Hypothesis D: Mindfulness will be associated with lower traumatic distress}

appraisal. Primary analyses showed that mindfulness was not associated with traumatic distress appraisal. This finding is inconsistent with other studies that have found a significant relationship between mindfulness and traumatic distress (e.g., Bormann,

Oman, Walter, \& Johnson, 2014; Kearney, Malte, et al., 2013; Kieviet-Stijnen, Visser, Garssen, \& Hudig, 2008). Traumatic distress appraisal was assessed using the summary score of variables that asked the participant "How traumatic was it?" This summary score was designed to create an aggregate score of cumulative life time traumatic distress appraisal (i.e. how severe did you find your lifetime traumatic events). This summary score, however, does not provide specific symptoms of traumatic distress including specific thoughts, feelings or behaviors associated with a potentially traumatic experience. Given that this measurement tool is meant to be a cognitive appraisal of overall trauma severity, it is potentially asking one to rate, in a metacognitive capacity, one's traumatic distress over the lifetime. As mindfulness has been purported to be a 
metacognitive capability, the non-significant finding between these two variables is both surprising and intriguing.

Mindfulness has been thought to be a mode of reperceiving thoughts to lead to an objectification or disidenitfication from mental contents; i.e. it can allow an individual to step back from their cognitions and allow for positive reappraisal (Garland, Gaylord, \& Park, 2009). Within the last year, research into differentiating the cognitive mechanisms of both dispositional mindfulness and acquired mindfulness has shown that there are unique differences in what each type of mindfulness does in the brain. Acquired mindfulness has been found to be more associated with the cognitive process of reappraisal (Garland, Hanley, Farb, \& Froeliger, 2015), while dispositional mindfulness has been more strongly linked to decentering and finding purpose in one's life (Pearson, Brown, Bravo, \& Witkiewitz, 2015), and emotional recognition and recovery (Fogarty et al., 2015). Given that this study has been designed to examine dispositional mindfulness as opposed to acquired mindfulness, this may explain the lack of significance between traumatic distress appraisal and dispositional mindfulness. Studies that have examined the association between mindfulness and traumatic distress appraisal have been largely longitudinal in nature (e.g., Kearney et al., 2012; King et al., 2013) and can better capture the process of reappraisal over time with acquired mindfulness. It may be that a followup study that includes a measure of acquired mindfulness post-intervention will be more likely to find a significant association.

Additionally, the wording of the measurement tool is vague and brings into question that it is actually assessing for traumatic distress appraisal. Is the tool actually measuring one's appraisal of distress associated with that particular event, as it was at the 
time of the event? In this regard, the question of "how traumatic was it" does not offer specificity or clarity on what constructs it is actually trying to assess for. It could be that this particular measurement is tapping into different constructs depending on how the participant interpreted the question. For example, it could be a measurement of appraisal of distress. Or it could be an assessment of how traumatic this particular event was in relation to other events. Or it could be a measurement of an aggregate of thoughts, feelings and emotions of how it felt at the time of the event. Or it could be measuring the perceived overall impact on one's life since the event. Given the vague and potentially open-ended question of traumatic distress appraisal, it makes it difficult to know what aspects of traumatic distress (emotions, cognitions, behaviors) the measurement tool is actually tapping into. This complicates the discussion of the results significantly. Hence, future work should include measurements of traumatic distress appraisal that are more specific, concise and clear.

\section{Hypothesis E: Mindfulness will be associated with lower cancer-specific}

distress. Primary analyses demonstrated that mindfulness was significantly associated with decreased cancer-specific distress. This finding is consistent with the literature that has examined this relationship in breast cancer (Tamagawa et al., 2013) and heterogeneous cancer populations (Bränström et al., 2010; Garland et al., 2013). This finding indicates that those with higher dispositional mindfulness have lower cancerspecific distress, demonstrating a possible buffering effect of developing PTSS following a cancer diagnosis. This finding may help explain the lower cancer-specific distress scores seen in this sample; however, this is speculative as this study was unable to measure distress scores over time since initial diagnosis. Future work should include 
longitudinal measurements of cancer-specific distress to see if those with increased dispositional mindfulness adjust and adaptively cope with their cancer experience faster than those with lower dispositional mindfulness.

Secondary analyses revealed that total mindfulness was negatively associated with all three subscales of cancer-specific distress (intrusions, hyperarousal and avoidance). All three subscales were highly significant, but the subscale of avoidance was the most significant in its association with mindfulness. This follows the theory that mindfulness serves to discourage the habitual avoidant reactions common in individuals experiencing PTSS. In regards to mindfulness subscales, only three subscales were associated with overall cancer-specific distress (describe, act with awareness and nonjudgment). These three subscales can be conceptualized as being facets of decentering, the ability to perceive thoughts and feelings as both impermanent and objective occurrences in the mind (Hofmann et al., 2011), an ability that has been strongly linked to depositional mindfulness and has been negatively associated with traumatic stress (Thompson, Arnkoff, \& Glass, 2011; Wilson, Smith, \& Johnson, 2013). In order to decenter from one's experiences, an individual must be able to objectively describe their experience, not judge one's reactions to these experience, and respond accordingly by acting with awareness. Having increased awareness and insight into one's experience may foster adaptive coping strategies and responsive choices as opposed to engaging in reflexive reactions. These three subscales denote the experience of decentering and provide further validity to the potential use of decentering for lung cancer patients with traumatic stress. 


\section{Hypothesis F: Mindfulness will be associated with higher HRQOL. As}

expected, primary analyses revealed that dispositional mindfulness was positively associated with HRQOL. This is consistent with the literature which has found this association in multiple cancer populations including breast cancer (Carlson, Speca, Patel, \& Goodey, 2003; Hoffman et al., 2012; Witek-Janusek et al., 2008), gynecologic cancer (Stafford et al., 2013), prostate cancer (Carlson et al., 2003) and lung cancer (Lehto, Wyatt, Sikorskii, Tesnjak, \& Kaufman, 2015). Dispositional mindfulness is associated with emotional regulation and finding meaning in one's life (Pearson et al., 2015), both of which have implications for HRQOL and well-being.

Secondary analyses demonstrated that mindfulness was associated with all subscales of HRQOL, excluding social and family well-being. Given that mindfulness is an internal process that is involved with aspects of decentering and emotion regulation, the external facet of social and family well-being might not be expected to be associated with mindfulness. Mindfulness may function as a way of reperceiving one's cancer experience and finding new meaning in life. As lung cancer is often diagnosed in later stages, as is seen in this sample, finding meaning and purpose at the end of life is an important component of well-being. It may be that mindfulness allows patients to decenter from their lung cancer experience and re-conceptualize what is important to focus on, which can engender well-being.

Secondary analyses also revealed that only two subscales were driving the significance between mindfulness and HRQOL: the describe and act with awareness subscales, both of which can be conceptualized as being part of emotion regulation and decentering. Mindfulness has been linked to greater emotional awareness which is 
predictive of increased HRQOL (Boden, Irons, Feldner, Bujarski, \& Bonn-Miller, 2014). Both subscales allow patients to effectively communicate with others by describing their experiences and then prompts purposeful actions with increased awareness. Further work should be done with larger sample sizes to tease apart how subscales of mindfulness correspond with subscales of HRQOL.

While metacognitive-based models are the most widely accepted theories for how mindfulness exerts it ameliorative impact, this dissertation provides support for the model proposed by Salmon, Sephton and Dreeben (see Figure 1; 2011). This model proposes that mindfulness functions to foster heightened awareness of potential stressors and promotes skillful responses to these stressors instead of engaging in reflexive distress (Salmon, Sephton \& Dreeben, 2011). This model proposed that mindfulness may promote well-being and health through several pathways, not just through metacognitive awareness. In this respect, mindfulness potentially exerts direct effects on several domains such as appraisal, emotional outcomes and health outcomes (Salmon, Sephton \& Dreeben, 2011). This model is in line with the mindfulness stress-buffering hypothesis that posits that mindfulness both mitigates stress appraisals and reduces stress reactivity (Creswell \& Lindsay, 2014). One study that examined this hypothesis found that healthy adults with higher dispositional mindfulness had lower psychological stress reactivity (Weinstein et al., 2009), lower anxiety and lower negative affect in response to a laboratory stress (Brown, Weinstein \& Creswell, 2012). The current study follows this trend as those with higher dispositional mindfulness had lower cancer-specific distress and higher HRQOL. 


\section{Aim 3. Explore mindfulness as a protective factor that may buffer}

associations between trauma and HRQOL (Hypotheses $\mathbf{G}$ and $\mathbf{H}$ ). This aim was exploratory and was proposed to begin elucidating the multifaceted construct of mindfulness and its role in promoting well-being following a cancer diagnosis. To evaluate mindfulness as a potential moderator of the relationship between trauma variables and HRQOL, assumptions of moderation were tested. For both hypotheses G and $\mathrm{H}$, the first assumption of moderation was violated as the potential moderating variable, mindfulness, was significantly correlated with HRQOL and cancer-specific distress. This association as not completely unexpected given the strong association between mindfulness and HRQOL found in the literature (e.g., Stafford et al., 2013; Witek-Janusek et al., 2008). However, these were primarily breast cancer samples, and these associations had not yet been demonstrated in lung cancer, making this exploratory aim worth considering. Therefore, tests of moderation were carried out in a fashion meant to be hypothesis generating for future work and should not be interpreted as hypothesis testing.

This study did not find support for the moderating effect of mindfulness on the relationship between trauma factors (traumatic distress appraisal or cancer-specific distress) and HRQOL. This negative finding raises the possibility that increased dispositional mindfulness does not exert a direct effect on the relationship between trauma and HRQOL, but rather it may be impactful through a series of other cognitive and affective changes that were not measured for this study. For example, it may be that people who are more mindful ruminate less, which may then impact the relationship between trauma and HRQOL. Future research should include measures of emotional 
regulation strategies, cognitive strategies and behavioral strategies that may be the mechanisms through which mindfulness exerts its impact through a possible model of moderated mediation.

Given the lack of consensus regarding the role of mindfulness as a dispositional trait or acquired state, further research that can lend support for one or the other is an avenue of future research. Paul Grossman (2014), one of the most prolific critics of mindfulness-based research, asserts that mindfulness does not exist as a dispositional trait, but instead is best captured by the definition of a mental process or state. While this may be the case, it may also be that our current measurement tools are unable to accurately capture the potential dispositional aspect of mindfulness, but instead lend to a more accurate measurement of acquired mindfulness. Future research should further elucidate not only the construct of mindfulness, but also how to best measure it to accurately capture the nuances of this complex construct.

\section{Strengths and Limitations}

There are several strengths of the current study. Research with lung cancer patients is novel. There is a paucity of research in the area of lung cancer and this study adds much needed data to a sparse field of study. This is the first study, to date, that examines the intersection of trauma variables, HRQOL and mindfulness within a sample of lung cancer patients. Furthermore, this study elucidates both vulnerability and protective factors that impact HRQOL for lung cancer patients. Enhancing HRQOL is a main treatment goal for those with later stage cancer diagnoses and this study provides further clarity into the psychosocial correlates that impact this outcome. 
This study also endeavored to further tease apart multifaceted constructs such as trauma and mindfulness. Three different measures of trauma were utilized, and how these variables all relate and contributed to decrements in well-being were examined separately. Additionally, mindfulness is a broad construct that has no gold-standard measurement tool, nor consensus on an operational definition by experts that study its impact on various outcomes. This dissertation attempted to further distinguish the nuances of the construct by offering a clear operational definition of both aspects of mindfulness as proposed by the literature. Further, the initial step in testing one conceptualization, dispositional mindfulness, in an exploratory model (e.g., moderation) was attempted.

Another strength of this study is in the demographics found within the sample. In contrast with the majority of the cancer literature that is predominantly focused on European American, female, highly educated breast cancer samples with higher incomes, this sample taps into demographics not typically found within the cancer research context. One-quarter of the sample identified as African American (21.7\%), were men (26.1\%), one-third reported low annual household income (30.6\%; less than \$20,000) and half of the sample had fewer than 12 years of education (57.8\%). The results of this study may be more generalizable to the diverse populations of individuals that are diagnosed with lung cancer.

The current study also has several limitations. This study has a small sample size and a large number of analyses were tested. This increases the potential for Type I error. Thus, the current study should be considered preliminary and viewed with caution in mind. 
There are several sampling biases that should be taken into account. The sample was self-selected, and it may be that patients that were in more distress were less likely to participate in the research (which may explain the lower cancer-specific distress scores found within this sample). Additionally, the sample includes individuals that have been diagnosed within the last five years which represents a large heterogeneity in the cancer experience depending on where the individual is within their cancer journey. A potential confounding variable that was not assessed in this study was participation in active treatment at the time of study enrollment. This is likely a large confounding variable that would potentially directly influence distress at the time of the study. Active chemotherapy and radiation can be painful and negatively impact HRQOL. Future work should assess for these variables and control for them. As this was not assessed during this study it is difficult to say how it may be impacting results. However, individuals that are not in active treatment may be more likely to participate in a research study, while those that are might decline. Another limitation is that the study relied exclusively on self-report measures, and the results are subject to biases inherent in utilizing such measurement tools.

An additional limitation of the study is that patients with different stages of disease, and phases of treatment were included within the sample. Future work may want to investigate the relationships of interest within more homogenous samples. One of the main limitations of the study is that it is cross-sectional by design. This cross-sectional study begins to address the relationships between trauma, HRQOL and mindfulness, but causal inferences and relationships can not be delineated due to the study design; a longitudinal study can begin to explore causal relationships in more detail. 


\section{Future Directions}

There are potential important research and clinical implications of the current findings that suggest promising leads for future research. As dispositional mindfulness was positively associated with decreased cancer-specific distress and increased HRQOL, a longitudinal follow-up study that assesses acquired mindfulness may further elucidate the debate of mindfulness as a trait or state. As this type of question has yet to be analyzed within the same sample, this follow-up analysis with the intervention data currently being collected within this study is needed within the literature. As the initial relationships between mindfulness and cancer-specific distress and HRQOL were so strong, it stands to reason that these patients would respond favorably to a mindfulnessbased intervention. A longitudinal follow-up analysis would be an appropriate next step to close the loop on how to best conceptualize mindfulness within a cancer context.

Another future avenue would be to test how aspects of mindfulness may be associated with different coping strategies. It has been shown that dispositional mindfulness is strongly associated with emotional regulation and finding meaning, while acquired mindfulness is associated with reappraisal. Having measurements of these constructs (mindfulness, emotional regulation, finding meaning and reappraisal) within the same sample, and then sampling both at baseline and follow-up may help to further explicate the differences between dispositional mindfulness and acquired mindfulness, and how they exert their impact on well-being.

Future research is needed with larger sample sizes. While medium to large effect sizes were found in this smaller sample, larger studies will be able to confirm complex 
effects that were initially shown within this data. These studies can then begin to inform a way to study potential mindfulness-based treatment avenues. Larger sample sizes will also allow for more complex models to be tested with the subscales of each measure, allowing for more definitive conclusions to be drawn regarding the potential mechanisms at work.

Future work may also benefit from understanding how participation in psychological treatment prior to study entry may impact results. The effects of traumatic distress appraisal on HRQOL are clear within this sample, but the question of prior psychological treatment history was not assessed. Future studies could assess for this variable and potentially discover a moderator of HRQOL through psychological treatment participation.

As mentioned above, having a clear measure of traumatic distress appraisal would be something to include in future work. The question utilized in this study was vague and open-ended, complicating the interpretation of the results. Choosing a measure of traumatic distress appraisal that includes more information, clearer instructions and has normative data on which to compare results to would make the results confirmatory and clear. In order to test how appraisal of traumatic events factors into these relationships, using a scale that was designed to measure this construct specifically should be utilized in future work. The Posttraumatic Cognitions Inventory, (PCTI; Foa, Ehlers, Clark, Tolin, \& Orsillo, 1999), for example, would be most appropriate to tap into this construct clearly. This scale was designed to assess negative trauma-specific cognitive appraisals and includes three factors: negative appraisal of self, negative appraisal of the world and appraisal of self-blame (Foa et al., 1999). This measure has been widely used in the 
literature and has strong reliability and validity, and would be an appropriate measure for the lung cancer patients as self-blame is often a common occurrence.

Future work should also assess the potential bidirectional relationships within the proposed model. The current model inherently proposes a linear understanding of variables impacting each other in a linear cascade. However, it is more likely that the model is bidirectional and cyclical in its actuality. For example, the question of how trauma history may impact the development of dispositional mindfulness is a question yet to answered in the literature. Although the question of temporal precedence has been assumed with the current definition of mindfulness, future studies should endeavor to test mindfulness prior to a cancer diagnosis to confirm if mindfulness can truly protect against cancer-specific distress. Perceived HRQOL may serve as a buffer to traumatic distress, as well, given the present moment, values-driven construct and its measurement of well-being. These potentially bidirectional relationships should be examined further in future work as they may further elucidate the nuanced presentation of HRQOL for lung cancer patients and better inform future interventions.

An additional follow-up study to this dissertation could be to examine the associations between the psychosocial variables examined here and physiological variables related to tumor growth. A deeper understanding of how psychological variables may be related to tumor growth and development may further inform potential intervention studies. Additionally, survival analyses conducted with these variables as predictors may further elucidate how these psychosocial correlates impact the cancer experience and further aid in the creation of appropriate intervention studies to promote HRQOL at the end of life. The potential to enhance HRQOL, survival, and/or reduce 
cancer recurrence rates represent only a few of the many possible outcomes of continued research into this area.

\section{Conclusions}

Little research has been done on the psychosocial aspects of lung cancer. This study presents preliminary information on the potential risk and protective factors that impact quality of life in a subset of this vulnerable population. The results demonstrate that there are significant relationships between trauma variables (as measured by traumatic distress appraisal and cancer-specific distress), HRQOL and dispositional mindfulness. The results of this study are limited by the cross-sectional design, preventing definitive conclusions to be determined. However, the results do indicate that dispositional mindfulness may have an ameliorative impact on adjustment to lung cancer. Further research is needed to determine the robustness of these findings in larger sample sizes, as well as how these relationships may differ in a longitudinal design that assesses for acquired mindfulness.

There is a pressing need to understand both the vulnerability and protective factors that impact HRQOL in lung cancer patients given the often later stage disease diagnosis. Learning what factors negatively impact (e.g., traumatic distress appraisal and cancer-specific distress) and what factors positively impact (e.g., dispositional mindfulness) HRQOL can inform future interventions that may help both the patients and family members confront the often traumatic experience of cancer diagnosis and treatment. Results from this study suggest that mindfulness represents one avenue for intervention. Additional research is needed to explore further the nature of mindfulness in 
the face of imminent death and how it might lead to peace for both patients and family members. Other psychological factors may be involved in this process and potential correlates should be examined in future work with hospice patients that may influence one's mindfulness including spirituality and sense of coherence.

The current findings highlight the importance of screening for both lifetime distress and cancer-specific distress in cancer patients as both have been seen to negatively impact HRQOL. This study demonstrates how dispositional mindfulness may promote well-being despite one's level of distress, and has important implications for designing future intervention studies. This has salient implications for both healthy and ill populations, but is particularly relevant in the context of lung cancer as HRQOL is considered a primary goal of treatment for this population. Notably, the present study suggests that dispositional mindfulness is a variable which may assist in selecting patients for the most appropriate psychosocial interventions to increase HRQOL, and is certainly worthy of further investigation. 


\section{REFERENCES}

Akechi, T., Taniguchi, K., Suzuki, S., Okamura, M., Minami, H., Okuyama, T., ... Uchitomi, Y. (2007). Multifaceted psychosocial intervention program for breast cancer patients after first recurrence: Feasibility study. PsychoOncology, 16(6), 517-524.

Allen, A. B., \& Leary, M. R. (2010). Self-Compassion, stress, and coping. Social and Personality Psychology Compass, 4(2), 107-118.

Alter, C. L., Pelcovitz, D., Axelrod, A., Goldenberg, B., Harris, H., Meyers, B., ... Kaplan, S. (1996). Identification of PTSD in cancer survivors. Psychosomatics, 37(2), $137-143$.

Altschuler, A., Rosenbaum, E., Gordon, P., Canales, S., \& Avins, A. L. (2012). Audio recordings of mindfulness-based stress reduction training to improve cancer patients' mood and quality of life-a pilot feasibility study. Supportive Care in Cancer, 20(6), 1291-1297. http://doi.org/10.1007/s00520-011-1216-7

American Cancer Society. (2016). Lung Cancer (Non-Small Cell). American Cancer Society. Retrieved from http://www.cancer.org/cancer/lungcancer-nonsmallcell/detailedguide/non-small-cell-lung-cancer-key-statistics

Amir, M., \& Ramati, A. (2002). Post-traumatic symptoms, emotional distress and quality of life in long-term survivors of breast cancer: a preliminary research. Journal of Anxiety Disorders, 16(2), 191-206. 
Ando, M., Morita, T., Akechi, T., Ito, S., Tanaka, M., Ifuku, Y., \& Nakayama, T. (2009).

The Efficacy of Mindfulness-Based Meditation Therapy on Anxiety, Depression, and Spirituality in Japanese Patients with Cancer. Journal of Palliative Medicine, 12(12), 1091-1094.

http://doi.org/10.1089/jpm.2009.0143

Andrykowski, M. A., Steffens, R. F., Bush, H. M., \& Tucker, T. C. (2015a). Lung Cancer Diagnosis and Treatment as a Traumatic Stressor in DSM-IV and DSM-5: Prevalence and Relationship to Mental Health Outcomes. Journal of Traumatic Stress. Retrieved from http://onlinelibrary.wiley.com/doi/10.1002/jts.22005/full

Andrykowski, M. A., Steffens, R. F., Bush, H. M., \& Tucker, T. C. (2015b). Lung Cancer Diagnosis and Treatment as a Traumatic Stressor in DSM-IV and DSM-5: Prevalence and Relationship to Mental Health Outcomes. Journal of Traumatic Stress. Retrieved from http://onlinelibrary.wiley.com/doi/10.1002/jts.22005/full

Antoni, M. H., Lutgendorf, S. K., Cole, S. W., Dhabhar, F. S., Sephton, S. E., McDonald, P. G., ... Sood, A. K. (2006). The influence of bio-behavioural factors on tumour biology: pathways and mechanisms. Nature Reviews Cancer, 6(3), 240-248.

Arch, J. J., \& Craske, M. G. (2010). Laboratory stressors in clinically anxious and nonanxious individuals: The moderating role of mindfulness. Behaviour Research and Therapy, 48(6), 495-505.

Association, A. P., \& others. (2013). Diagnostic and statistical manual of mental disorders (DSM-5®). American Psychiatric Pub. Retrieved from 
https://books.google.com/books?hl=en\&lr=\&id=-

JivBAAAQBAJ\&oi=fnd\&pg=PT18\&dq=DSM-5\&ots=cdT05-

NGt9\&sig=wUDKe9rsynp8bGpN_E2htiEvPjc

Babyak, M. A. (2004). What you see may not be what you get: a brief, nontechnical introduction to overfitting in regression-type models. Psychosomatic Medicine, 66(3), 411-421.

Baer, R. A. (2003a). Mindfulness training as a clinical intervention: A conceptual and empirical review. Clinical Psychology: Science and Practice, 10(2), 125-143.

Baer, R. A. (2003b). Mindfulness training as a clinical intervention: A conceptual and empirical review. Clinical Psychology: Science and Practice, 10(2), 125-143.

Baer, R. A., Smith, G. T., Hopkins, J., Krietemeyer, J., \& Toney, L. (2006). Using selfreport assessment methods to explore facets of mindfulness. Assessment, 13(1), 27-45.

Banou, E., Hobfoll, S. E., \& Trochelman, R. D. (2009). Loss of resources as mediators between interpersonal trauma and traumatic and depressive symptoms among women with cancer. Journal of Health Psychology, 14(2), 200-214.

Banthia, R., Malcarne, V. L., Varni, J. W., Ko, C. M., Sadler, G. R., \& Greenbergs, H. L. (2003). The effects of dyadic strength and coping styles on psychological distress in couples faced with prostate cancer. Journal of Behavioral Medicine, 26(1), 31-52.

Baron, R. M., \& Kenny, D. A. (1986). The moderator-mediator variable distinction in social psychological research: Conceptual, strategic, and statistical considerations. Journal of Personality and Social Psychology, 51(6), 1173. 
Becker, C. B., \& Zayfert, C. (2001). Integrating DBT-based techniques and concepts to facilitate exposure treatment for PTSD. Cognitive and Behavioral Practice, $8(2), 107-122$.

Bernstein, A., Tanay, G., \& Vujanovic, A. A. (2011). Concurrent relations between mindful attention and awareness and psychopathology among traumaexposed adults: Preliminary evidence of transdiagnostic resilience. Journal of Cognitive Psychotherapy, 25(2), 99-113.

Berterö, C., Vanhanen, M., \& Appelin, G. (2008). Receiving a diagnosis of inoperable lung cancer: patients' perspectives of how it affects their life situation and quality of life. Acta Oncologica, 47(5), 862-869.

Birnie, K., Speca, M., \& Carlson, L. E. (2010). Exploring self-compassion and empathy in the context of mindfulness-based stress reduction (MBSR). Stress and Health, 26(5), 359-371.

Bishop, S. R., Lau, M., Shapiro, S., Carlson, L., Anderson, N. D., Carmody, J., ... others. (2004). Mindfulness: A proposed operational definition. Clinical Psychology: Science and Practice, 11(3), 230-241.

Boden, M. T., Bernstein, A., Walser, R. D., Bui, L., Alvarez, J., \& Bonn-Miller, M. 0. (2012). Changes in facets of mindfulness and posttraumatic stress disorder treatment outcome. Psychiatry Research, 200(2), 609-613.

Boden, M. T., Irons, J. G., Feldner, M. T., Bujarski, S., \& Bonn-Miller, M. O. (2014). An Investigation of Relations Among Quality of Life and Individual Facets of Emotional Awareness and Mindfulness. Mindfulness, 6(4), 700-707. http://doi.org/10.1007/s12671-014-0308-0 
Bormann, J. E., Oman, D., Walter, K. H., \& Johnson, B. D. (2014a). Mindful attention increases and mediates psychological outcomes following mantram repetition practice in Veterans with posttraumatic stress disorder. Medical Care, 52, S13-S18.

Bormann, J. E., Oman, D., Walter, K. H., \& Johnson, B. D. (2014b). Mindful attention increases and mediates psychological outcomes following mantram repetition practice in Veterans with posttraumatic stress disorder. Medical Care, 52, S13-S18.

Bränström, R., Kvillemo, P., Brandberg, Y., \& Moskowitz, J. T. (2010). Self-report Mindfulness as a Mediator of Psychological Well-being in a Stress Reduction Intervention for Cancer Patients-A Randomized Study. Annals of Behavioral Medicine, 39(2), 151-161. http://doi.org/10.1007/s12160-010-9168-6

Brewin, C. R., \& Holmes, E. A. (2003). Psychological theories of posttraumatic stress disorder. Clinical Psychology Review, 23(3), 339-376.

Browning, K. K., Ferketich, A. K., Otterson, G. A., Reynolds, N. R., \& Wewers, M. E. (2009). A psychometric analysis of quality of life tools in lung cancer patients who smoke. Lung Cancer, 66(1), 134-139.

Brown, K. W., \& Ryan, R. M. (2003). The benefits of being present: mindfulness and its role in psychological well-being. Journal of Personality and Social Psychology, 84(4), 822.

Brown, K. W., Ryan, R. M., \& Creswell, J. D. (2007). Mindfulness: Theoretical foundations and evidence for its salutary effects. Psychological Inquiry, 18(4), 211-237. 
Brown, K. W., Weinstein, N., \& Creswell, J. D. (2012a). Trait mindfulness modulates neuroendocrine and affective responses to social evaluative threat. Psychoneuroendocrinology, 37(12), 2037-2041.

Brown, K. W., Weinstein, N., \& Creswell, J. D. (2012b). Trait mindfulness modulates neuroendocrine and affective responses to social evaluative threat. Psychoneuroendocrinology, 37(12), 2037-2041.

Button, K. S., Ioannidis, J. P., Mokrysz, C., Nosek, B. A., Flint, J., Robinson, E. S., \& Munafò, M. R. (2013). Power failure: why small sample size undermines the reliability of neuroscience. Nature Reviews Neuroscience, 14(5), 365-376.

Camps, C., del Pozo, N., Blasco, A., Blasco, P., \& Sirera, R. (2009). Importance of quality of life in patients with non-small-cell lung cancer. Clinical Lung Cancer, 10(2), 83-90.

Carlsen, K., Jensen, A. B., Jacobsen, E., Krasnik, M., \& Johansen, C. (2005). Psychosocial aspects of lung cancer. Lung Cancer, 47(3), 293-300.

Carlson, K. J., Silva, S. G., Langley, J., \& Johnson, C. (2013). Mindful-veteran: the implementation of a brief stress reduction course. Complementary Therapies in Clinical Practice, 19(2), 89-96.

Carlson, L. E., \& Garland, S. N. (2005). Impact of mindfulness-based stress reduction (MBSR) on sleep, mood, stress and fatigue symptoms in cancer outpatients. International Journal of Behavioral Medicine, 12(4), 278-285.

Carlson, L. E., Speca, M., Patel, K. D., \& Goodey, E. (2003a). Mindfulness-based stress reduction in relation to quality of life, mood, symptoms of stress, and 
immune parameters in breast and prostate cancer outpatients. Psychosomatic Medicine, 65(4), 571-581.

Carlson, L. E., Speca, M., Patel, K. D., \& Goodey, E. (2003b). Mindfulness-based stress reduction in relation to quality of life, mood, symptoms of stress, and immune parameters in breast and prostate cancer outpatients. Psychosomatic Medicine, 65(4), 571-581.

Carlson, L. E., Speca, M., \& Segal, Z. V. (2010). Mindfulness-based cancer recovery. Oakland, CA: New Harbinger. Retrieved from http://www.oncologyex.com/pdf/vol12_no2/feature-mindfulness-basedcancer-recovery.pdf

Casso, D., Buist, D. S., \& Taplin, S. (2004). Quality of life of 5-10 year breast cancer survivors diagnosed between age 40 and 49. Health and Quality of Life Outcomes, 2(1), 25.

Cataldo, J. K., Slaughter, R., Jahan, T. M., Pongquan, V. L., \& Hwang, W. J. (2011). Measuring stigma in people with lung cancer: psychometric testing of the cataldo lung cancer stigma scale. In Oncology nursing forum (Vol. 38, p. E46). NIH Public Access. Retrieved from http://www.ncbi.nlm.nih.gov/pmc/articles/PMC3182474/

Cella, D. F., Bonomi, A. E., Lloyd, S. R., Tulsky, D. S., Kaplan, E., \& Bonomi, P. (1995). Reliability and validity of the Functional Assessment of Cancer TherapyLung (FACT-L) quality of life instrument. Lung Cancer, 12(3), 199-220.

Chambers, S. K., Foley, E., Galt, E., Ferguson, M., \& Clutton, S. (2012). Mindfulness groups for men with advanced prostate cancer: a pilot study to assess 
feasibility and effectiveness and the role of peer support. Supportive Care in Cancer, 20(6), 1183-1192. http://doi.org/10.1007/s00520-011-1195-8

Chang, N.-W., Lin, K.-C., Hsu, W.-H., Lee, S.-C., Chan, J. Y.-H., \& Wang, K.-Y. (2015). The effect of gender on health-related quality of life and related factors in postlobectomy lung-cancer patients. European Journal of Oncology Nursing, 19(3), 292-300. http://doi.org/10.1016/j.ejon.2014.10.015

Cohen, S., Janicki-Deverts, D., \& Miller, G. E. (2007). Psychological stress and disease. Jama, 298(14), 1685-1687.

Cramer, H., Lauche, R., Paul, A., \& Dobos, G. (2012). Mindfulness-based stress reduction for breast cancer--a systematic review and meta-analysis. Current Oncology, 19(5), e343-e352. http://doi.org/10.3747/co.19.1016

Creamer, M., Bell, R., \& Failla, S. (2003). Psychometric properties of the impact of event scale-revised. Behaviour Research and Therapy, 41(12), 1489-1496.

Creswell, J. D. (2014). Biological pathways linking mindfulness with health. Handbook of Mindfulness: Theory, Research, and Practice. Guilford Press, New York, NY. Retrieved from http://www.psy.cmu.edu/ creswell/papers/Creswell\%20(2014),\%20mindf ulness $\% 20$ stress $\% 20$ buffering, $\% 20$ chp.pdf

Creswell, J. D., \& Lindsay, E. K. (2014). How Does Mindfulness Training Affect Health? A Mindfulness Stress Buffering Account. Current Directions in Psychological Science, 23(6), 401-407. http://doi.org/10.1177/0963721414547415 
Creswell, J. D., Pacilio, L. E., Lindsay, E. K., \& Brown, K. W. (2014). Brief mindfulness meditation training alters psychological and neuroendocrine responses to social evaluative stress. Psychoneuroendocrinology, 44, 1-12.

Curtiss, J., \& Klemanski, D. H. (2014). Factor analysis of the five facet mindfulness questionnaire in a heterogeneous clinical sample. Journal of Psychopathology and Behavioral Assessment, 36(4), 683-694.

DePrince, A. P., Chu, A. T., \& Pineda, A. S. (2011). Links between specific posttrauma appraisals and three forms of trauma-related distress. Psychological Trauma: Theory, Research, Practice, and Policy, 3(4), 430.

Dorahy, M. J. (2010). The impact of dissociation, shame, and guilt on interpersonal relationships in chronically traumatized individuals: A pilot study*. Journal of Traumatic Stress, 23(5), 653-656.

DuHamel, K. N., Smith, M. Y., Vickberg, S. M. J., Papadopoulos, E., Ostroff, J., Winkel, G., ... Redd, W. H. (2001). Trauma symptoms in bone marrow transplant survivors: The role of nonmedical life events. Journal of Traumatic Stress, 14(1), 95-113.

Ehlers, A., \& Clark, D. M. (2000). A cognitive model of posttraumatic stress disorder. Behaviour Research and Therapy, 38(4), 319-345.

Ellis, A. A., Nixon, R. D., \& Williamson, P. (2009). The effects of social support and negative appraisals on acute stress symptoms and depression in children and adolescents. British Journal of Clinical Psychology, 48(4), 347-361.

Fairchild, A. J., \& MacKinnon, D. P. (2009). A general model for testing mediation and moderation effects. Prevention Science, 10(2), 87-99. 
Fallah, R., Keshmir, F., Kashani, F. L., Azargashb, E., \& Akbari, M. E. (2012). Posttraumatic growth in breast cancer patients: a qualitative phenomenological study. Middle East Journal of Cancer, 3(2 \& 3), 35-44.

Foa, E. B., Ehlers, A., Clark, D. M., Tolin, D. F., \& Orsillo, S. M. (1999). The posttraumatic cognitions inventory (PTCI): Development and validation. Psychological Assessment, 11(3), 303.

Fogarty, F. A., Lu, L. M., Sollers III, J. J., Krivoschekov, S. G., Booth, R. J., \& Consedine, N. S. (2015). Why it pays to be mindful: Trait mindfulness predicts physiological recovery from emotional stress and greater differentiation among negative emotions. Mindfulness, 6(2), 175-185.

Follette, V. M., Palm, K. M., \& Hall, M. L. R. (2004). Acceptance, Mindfulness, and Trauma. Retrieved from http://psycnet.apa.org/psycinfo/2005-02461-009

Follette, V., Palm, K. M., \& Pearson, A. N. (2006a). Mindfulness and trauma: Implications for treatment. Journal of Rational-Emotive and CognitiveBehavior Therapy, 24(1), 45-61.

Follette, V., Palm, K. M., \& Pearson, A. N. (2006b). Mindfulness and trauma: Implications for treatment. Journal of Rational-Emotive and CognitiveBehavior Therapy, 24(1), 45-61.

Forbes, D., Creamer, M., \& Biddle, D. (2001). The validity of the PTSD checklist as a measure of symptomatic change in combat-related PTSD. Behaviour Research and Therapy, 39(8), 977-986.

Garland, E., Gaylord, S., \& Park, J. (2009). The role of mindfulness in positive reappraisal. Explore: The Journal of Science and Healing, 5(1), 37-44. 
Garland, E. L., Hanley, A., Farb, N. A., \& Froeliger, B. (2013). State Mindfulness During Meditation Predicts Enhanced Cognitive Reappraisal. Mindfulness, 6(2), 234242. http://doi.org/10.1007/s12671-013-0250-6

Garland, E. L., Hanley, A., Farb, N. A., \& Froeliger, B. (2015). State mindfulness during meditation predicts enhanced cognitive reappraisal. Mindfulness, 6(2), 234242.

Garland, S. N., Campbell, T., Samuels, C., \& Carlson, L. E. (2013). Dispositional mindfulness, insomnia, sleep quality and dysfunctional sleep beliefs in posttreatment cancer patients. Personality and Individual Differences, 55(3), 306311.

Garland, S. N., Tamagawa, R., Todd, S. C., Speca, M., \& Carlson, L. E. (2013). Increased mindfulness is related to improved stress and mood following participation in a mindfulness-based stress reduction program in individuals with cancer. Integrative Cancer Therapies, 12(1), 31-40.

Geffen, D. B., Blaustein, A., Amir, M., \& Cohen, Y. (2003). Post-traumatic stress disorder and quality of life in long-term survivors of Hodgkin's disease and non-Hodgkin's lymphoma in Israel. Leukemia \& Lymphoma, 44(11), 19251929.

Gilbert, P., \& Procter, S. (2006). Compassionate mind training for people with high shame and self-criticism: Overview and pilot study of a group therapy approach. Clinical Psychology \& Psychotherapy, 13(6), 353-379. 
Glinder, J. G., \& Compas, B. E. (1999). Self-blame attributions in women with newly diagnosed breast cancer: A prospective study of psychological adjustment. Health Psychology, 18(5), 475.

Golden-Kreutz, D. M., Thornton, L. M., Wells-Di Gregorio, S., Frierson, G. M., Jim, H. S., Carpenter, K. M., ... Andersen, B. L. (2005). Traumatic stress, perceived global stress, and life events: prospectively predicting quality of life in breast cancer patients. Health Psychology, 24(3), 288.

Gold, J. I., Douglas, M. K., Thomas, M. L., Elliott, J. E., Rao, S. M., \& Miaskowski, C. (2012). The relationship between posttraumatic stress disorder, mood states, functional status, and quality of life in oncology outpatients. Journal of Pain and Symptom Management, 44(4), 520-531.

Goldsmith, R. E., Gerhart, J. I., Chesney, S. A., Burns, J. W., Kleinman, B., \& Hood, M. M. (2014). Mindfulness-Based Stress Reduction for Posttraumatic Stress Symptoms Building Acceptance and Decreasing Shame. Journal of EvidenceBased Complementary \& Alternative Medicine, 19(4), 227-234.

Gonçalves, V., Jayson, G., \& Tarrier, N. (2011). A longitudinal investigation of posttraumatic stress disorder in patients with ovarian cancer. Journal of Psychosomatic Research, 70(5), 422-431.

Green, B. L. (1996). Trauma history questionnaire. Measurement of Stress, Trauma, and Adaptation, 1, 366-369.

Green, B. L., Krupnick, J. L., Rowland, J. H., Epstein, S. A., Stockton, P., Spertus, I., \& Stern, N. (2000). Trauma history as a predictor of psychologic symptoms in women with breast cancer. Journal of Clinical Oncology, 18(5), 1084-1084. 
Grossman, P. (2011). Defining mindfulness by how poorly I think I pay attention during everyday awareness and other intractable problems for psychology's (re) invention of mindfulness: comment on Brown et al.(2011). Retrieved from http://psycnet.apa.org/journals/pas/23/4/1034/

Grossman, P. (2014). Mindfulness: Awareness Informed by an Embodied Ethic. Mindfulness, 6(1), 17-22. http://doi.org/10.1007/s12671-014-0372-5

Grossman, P., Niemann, L., Schmidt, S., \& Walach, H. (2004). Mindfulness-based stress reduction and health benefits: A meta-analysis. Journal of Psychosomatic Research, 57(1), 35-43.

Gurevich, M., Devins, G. M., \& Rodin, G. M. (2002). Stress response syndromes and cancer: conceptual and assessment issues. Psychosomatics, 43(4), 259-281.

Guyatt, G. H., Feeny, D. H., \& Patrick, D. L. (1993). Measuring health-related quality of life. Annals of Internal Medicine, 118(8), 622-629.

Hawley, L. L., Schwartz, D., Bieling, P. J., Irving, J., Corcoran, K., Farb, N. A., ... Segal, Z. V. (2014). Mindfulness practice, rumination and clinical outcome in mindfulness-based treatment. Cognitive Therapy and Research, 38(1), 1-9.

Heim, C., Newport, D. J., Mletzko, T., Miller, A. H., \& Nemeroff, C. B. (2008). The link between childhood trauma and depression: insights from HPA axis studies in humans. Psychoneuroendocrinology, 33(6), 693-710.

Hoffman, C. J., Ersser, S. J., Hopkinson, J. B., Nicholls, P. G., Harrington, J. E., \& Thomas, P. W. (2012a). Effectiveness of mindfulness-based stress reduction in mood, breast-and endocrine-related quality of life, and well-being in stage 0 to III 
breast cancer: a randomized, controlled trial. Journal of Clinical Oncology, 30(12), 1335-1342.

Hoffman, C. J., Ersser, S. J., Hopkinson, J. B., Nicholls, P. G., Harrington, J. E., \& Thomas, P. W. (2012b). Effectiveness of mindfulness-based stress reduction in mood, breast-and endocrine-related quality of life, and well-being in stage 0 to III breast cancer: a randomized, controlled trial. Journal of Clinical Oncology, 30(12), 1335-1342.

Hofmann, S. G., Glombiewski, J. A., Asnaani, A., \& Sawyer, A. T. (2011). Mindfulness and acceptance: The perspective of cognitive therapy. Acceptance and Mindfulness in Cognitive Behavior Therapy: Understanding and Applying the New Therapies, 265-290.

Holland, J. C., \& Lewis, S. (2000). The human side of cancer: Living with hope, coping with uncertainty. HarperCollins New York. Retrieved from http://www.priory.com/ital/psico-oncologia/holland2.htm

Hwang, S. S., Chang, V. T., Fairclough, D. L., Cogswell, J., \& Kasimis, B. (2003). Longitudinal quality of life in advanced cancer patients: pilot study results from a VA medical cancer center. Journal of Pain and Symptom Management, 25(3), 225-235.

Iwatani, T., Matsuda, A., Kawabata, H., Miura, D., \& Matsushima, E. (2013). Predictive factors for psychological distress related to diagnosis of breast cancer. Psycho-Oncology, 22(3), 523-529.

Jacobsen, P. B., Widows, M. R., Hann, D. M., Andrykowski, M. A., Kronish, L. E., \& Fields, K. K. (1998). Posttraumatic stress disorder symptoms after bone 
marrow transplantation for breast cancer. Psychosomatic Medicine, 60(3), 366-371.

Jahn, A. L., Herman, L., Schuster, J., Naik, A., \& Moye, J. (2012). Distress and resilience after cancer in veterans. Research in Human Development, 9(3), 229-247.

Kabat-Zinn, J. (1982). An outpatient program in behavioral medicine for chronic pain patients based on the practice of mindfulness meditation: Theoretical considerations and preliminary results. General Hospital Psychiatry, 4(1), 3347.

Kabat-Zinn, J. (1990). Full catastrophe living: The program of the stress reduction clinic at the University of Massachusetts Medical Center. New York: Delta. Kabat-Zinn, J. (2003). Mindfulness-based interventions in context: past, present, and future. Clinical Psychology: Science and Practice, 10(2), 144-156.

Kangas, M., Henry, J. L., \& Bryant, R. A. (2002). Posttraumatic stress disorder following cancer: A conceptual and empirical review. Clinical Psychology Review, 22(4), 499-524.

Kangas, M., Williams, J. R., \& Smee, R. I. (2012). The association between posttraumatic stress and health-related quality of life in adults treated for a benign meningioma. Applied Research in Quality of Life, 7(2), 163-182.

Kang, C., \& Whittingham, K. (2010). Mindfulness: A dialogue between Buddhism and clinical psychology. Mindfulness, 1(3), 161-173.

Kang, D.-H., Park, N.-J., \& McArdle, T. (2012). Cancer-specific stress and mood disturbance: implications for symptom perception, quality of life, and immune response in women shortly after diagnosis of breast cancer. 
International Scholarly Research Notices, 2012. Retrieved from http://www.hindawi.com/journals/isrn/2012/608039/abs/

Kearney, D. J., Malte, C. A., McManus, C., Martinez, M. E., Felleman, B., \& Simpson, T. L. (2013a). Loving-Kindness Meditation for Posttraumatic Stress Disorder: A Pilot Study. Journal of Traumatic Stress, 26(4), 426-434.

Kearney, D. J., Malte, C. A., McManus, C., Martinez, M. E., Felleman, B., \& Simpson, T. L. (2013b). Loving-Kindness Meditation for Posttraumatic Stress Disorder: A Pilot Study. Journal of Traumatic Stress, 26(4), 426-434.

Kearney, D. J., McDermott, K., Malte, C., Martinez, M., \& Simpson, T. L. (2012). Association of participation in a mindfulness program with measures of PTSD, depression and quality of life in a veteran sample. Journal of Clinical Psychology, 68(1), 101-116.

Kearney, D. J., McDermott, K., Malte, C., Martinez, M., \& Simpson, T. L. (2013). Effects of participation in a mindfulness program for veterans with posttraumatic stress disorder: A randomized controlled pilot study. Journal of Clinical Psychology, 69(1), 14-27.

Kearney, D. J., McManus, C., Malte, C. A., Martinez, M. E., Felleman, B., \& Simpson, T. L. (2014). Loving-Kindness Meditation and the Broaden-and-Build Theory of Positive Emotions Among Veterans With Posttraumatic Stress Disorder. Medical Care, 52, S32-S38.

Khanna, D., \& Tsevat, J. (2007). Health-related quality of life-an introduction. The American Journal of Managed Care, 13, S218-23. 
Kieviet-Stijnen, A., Visser, A., Garssen, B., \& Hudig, W. (2008). Mindfulness-based stress reduction training for oncology patients: Patients' appraisal and changes in well-being. Patient Education and Counseling, 72(3), 436-442.

Kimbrough, E., Magyari, T., Langenberg, P., Chesney, M., \& Berman, B. (2010). Mindfulness intervention for child abuse survivors. Journal of Clinical Psychology, 66(1), 17-33.

Kim, H.-Y. (2013). Statistical notes for clinical researchers: assessing normal distribution (2) using skewness and kurtosis. Restorative Dentistry \& Endodontics, 38(1), 52-54. http://doi.org/10.5395/rde.2013.38.1.52

King, A. P., Erickson, T. M., Giardino, N. D., Favorite, T., Rauch, S. A., Robinson, E., ... Liberzon, I. (2013). A Pilot Study Of Group Mindfulness-Based Cognitive Therapy (Mbct) For Combat Veterans With Posttraumatic Stress Disorder (Ptsd). Depression and Anxiety, 30(7), 638-645.

Koopman, C., Butler, L. D., Classen, C., Giese-Davis, J., Morrow, G. R., Westendorf, J., ... Spiegel, D. (2002). Traumatic stress symptoms among women with recently diagnosed primary breast cancer. Journal of Traumatic Stress, 15(4), 277287.

Kraemer, H. C., Stice, E., Kazdin, A., Offord, D., \& Kupfer, D. (2001). How do risk factors work together? Mediators, moderators, and independent, overlapping, and proxy risk factors. American Journal of Psychiatry. Retrieved from http://ajp.psychiatryonline.org/doi/abs/10.1176/appi.ajp.158.6.848 
Kuyken, W., Watkins, E., Holden, E., White, K., Taylor, R. S., Byford, S., ... Dalgleish, T. (2010). How does mindfulness-based cognitive therapy work? Behaviour Research and Therapy, 48(11), 1105-1112.

Lanius, R. A., Vermetten, E., \& Pain, C. (2010). The impact of early life trauma on health and disease: The hidden epidemic. Cambridge University Press. Retrieved from https://books.google.com/books?hl=en\&lr=\&id=121nQqryvbkC\&oi=fnd\&pg $=$ PR5\&dq=trauma + impact + on + health\&ots=gH6WVnaDd2\&sig=DqdES87qDlt 48tntzIQACIUHHXQ

Lannin, D. R., Mathews, H. F., Mitchell, J., Swanson, M. S., Swanson, F. H., \& Edwards, M. S. (1998). Influence of socioeconomic and cultural factors on racial differences in late-stage presentation of breast cancer. Jama, 279(22), 18011807.

Lau, M. A., Bishop, S. R., Segal, Z. V., Buis, T., Anderson, N. D., Carlson, L., ... Devins, G. (2006). The Toronto mindfulness scale: Development and validation. Journal of Clinical Psychology, 62(12), 1445-1467.

Lehto, R. H., Wyatt, G., Sikorskii, A., Tesnjak, I., \& Kaufman, V. H. (2015). Home-based mindfulness therapy for lung cancer symptom management: a randomized feasibility trial. Psycho-Oncology, 24(9), 1208-1212. http://doi.org/10.1002/pon.3755

Linden, W., Vodermaier, A., MacKenzie, R., \& Greig, D. (2012). Anxiety and depression after cancer diagnosis: Prevalence rates by cancer type, gender, and age. Journal of Affective Disorders, 141(2), 343-351. 
Linehan, M. M. (1993). Skills training manual for treating borderline personality disorder. Guilford Press. Retrieved from http://psycnet.apa.org/psycinfo/1995-98090-000

Linehan, M. M., Tutek, D. A., Heard, H. L., \& Armstrong, H. E. (1994). Interpersonal outcome of cognitive behavioral treatment for chronically suicidal borderline patients. American Journal of Psychiatry, 151(12), 1771-1775.

Lutgendorf, S. K., Anderson, B., Rothrock, N., Buller, R. E., Sood, A. K., \& Sorosky, J. I. (2000). Quality of life and mood in women receiving extensive chemotherapy for gynecologic cancer. Cancer, 89(6), 1402-1411. http://doi.org/10.1002/1097-0142(20000915)89:6<1402::AIDCNCR26>3.0.CO;2-H

Lutgendorf, S. K., Slavich, G. M., DeGeest, K., Goodheart, M., Bender, D., Thaker, P. H., ... others. (2013). Non-cancer life stressors contribute to impaired quality of life in ovarian cancer patients. Gynecologic Oncology, 131(3), 667-673.

Lutz, A., Brefczynski-Lewis, J., Johnstone, T., \& Davidson, R. J. (2008). Regulation of the neural circuitry of emotion by compassion meditation: effects of meditative expertise. PloS One, 3(3), e1897.

Martin, C. G., Cromer, L. D., DePrince, A. P., \& Freyd, J. J. (2013). The Role of Cumulative Trauma, Betrayal, and Appraisals in Understanding Trauma Symptomatology. Psychological Trauma : Theory, Research, Practice and Policy, 52(2), 110-118. http://doi.org/10.1037/a0025686 
Matchim, Y., Armer, J. M., \& Stewart, B. R. (2010). Effects of mindfulness-based stress reduction (MBSR) on health among breast cancer survivors. Western Journal of Nursing Research, 0193945910385363.

Mehnert, A., Brähler, E., Faller, H., Härter, M., Keller, M., Schulz, H., ... others. (2014). Four-Week Prevalence of Mental Disorders in Patients With Cancer Across Major Tumor Entities. Journal of Clinical Oncology, JCO-2014.

Michal, M., Beutel, M. E., Jordan, J., Zimmermann, M., Wolters, S., \& Heidenreich, T. (2007). Depersonalization, mindfulness, and childhood trauma. The Journal of Nervous and Mental Disease, 195(8), 693-696.

Mystakidou, K., Parpa, E., Tsilika, E., Panagiotou, I., Roumeliotou, A., Galanos, A., \& Gouliamos, A. (2012). Traumatic Experiences of Patients With Advanced Cancer. Journal of Loss and Trauma, 17(2), 125-136.

Neff, K. (2003). Self-compassion: An alternative conceptualization of a healthy attitude toward oneself. Self and Identity, 2(2), 85-101.

Nipp, R. D., Greer, J. A., El-Jawahri, A., Traeger, L., Gallagher, E. R., Park, E. R., ... Temel, J. S. (2016). Age and Gender Moderate the Impact of Early Palliative Care in Metastatic Non-Small Cell Lung Cancer. The Oncologist, 21(1), 119126.

Nyklíček, I., Mommersteeg, P., Van Beugen, S., Ramakers, C., \& Van Boxtel, G. J. (2013). Mindfulness-based stress reduction and physiological activity during acute stress: a randomized controlled trial. Health Psychology, 32(10), 1110.

Okamura, M., Yamawaki, S., Akechi, T., Taniguchi, K., \& Uchitomi, Y. (2005).

Psychiatric disorders following first breast cancer recurrence: prevalence, 
associated factors and relationship to quality of life. Japanese Journal of Clinical Oncology, 35(6), 302-309.

Orsillo, S. M., \& Batten, S. V. (2005). Acceptance and commitment therapy in the treatment of posttraumatic stress disorder. Behavior Modification, 29(1), 95129.

Ott, M. J., Norris, R. L., \& Bauer-Wu, S. M. (2006). Mindfulness meditation for oncology patients: a discussion and critical review. Integrative Cancer Therapies, 5(2), 98-108.

Palesh, O., Butler, L. D., Koopman, C., Giese-Davis, J., Carlson, R., \& Spiegel, D. (2007). Stress history and breast cancer recurrence. Journal of Psychosomatic Research, 63(3), 233-239.

Pearson, M. R., Brown, D. B., Bravo, A. J., \& Witkiewitz, K. (2014). Staying in the Moment and Finding Purpose: The Associations of Trait Mindfulness, Decentering, and Purpose in Life with Depressive Symptoms, Anxiety Symptoms, and Alcohol-Related Problems. Mindfulness, 6(3), 645-653. http://doi.org/10.1007/s12671-014-0300-8

Pearson, M. R., Brown, D. B., Bravo, A. J., \& Witkiewitz, K. (2015). Staying in the moment and finding purpose: the associations of trait mindfulness, decentering, and purpose in life with depressive symptoms, anxiety symptoms, and alcohol-related problems. Mindfulness, 6(3), 645-653.

Penson, D. F., Stoddard, M. L., Pasta, D. J., Lubeck, D. P., Flanders, S. C., \& Litwin, M. S. (2001). The association between socioeconomic status, health insurance 
coverage, and quality of life in men with prostate cancer. Journal of Clinical Epidemiology, 54(4), 350-358.

Piet, J., Würtzen, H., \& Zachariae, R. (2012). The effect of mindfulness-based therapy on symptoms of anxiety and depression in adult cancer patients and survivors: A systematic review and meta-analysis. Journal of Consulting and Clinical Psychology, 80(6), 1007.

Rash, C. J., Coffey, S. F., Baschnagel, J. S., Drobes, D. J., \& Saladin, M. E. (2008). Psychometric properties of the IES-R in traumatized substance dependent individuals with and without PTSD. Addictive Behaviors, 33(8), 1039-1047.

Ristvedt, S. L., \& Trinkaus, K. M. (2009). Trait anxiety as an independent predictor of poor health-related quality of life and post-traumatic stress symptoms in rectal cancer. British Journal of Health Psychology, 14(4), 701-715. http://doi.org/10.1348/135910708X400462

Robins, C. J., Keng, S.-L., Ekblad, A. G., \& Brantley, J. G. (2012). Effects of mindfulnessbased stress reduction on emotional experience and expression: a randomized controlled trial. Journal of Clinical Psychology, 68(1), 117-131.

Rodin, G., Craven, J., \& Littlefield, C. (1991). Depression in the medically ill: an integrated approach. Psychology Press. Retrieved from https://books.google.com/books?hl=en\&lr=\&id=crQGP0e4PJIC\&oi=fnd\&pg= PR9\&dq=Rodin + G,+Craven+J,+Littlefield + C:+Depression + in + the + Medically + I ll:+An+Integrated+Approach.+New+York,+Brunner/Mazel,+1991\&ots=nBRA 0pet2u\&sig=PhgPpRIKAjoXI0ZKjsWOFtyfB6o 
Salmon, P. G., Sephton, S. E., \& Dreeben, S. J. (2011). Mindfulness-Based Stress Reduction. Acceptance and Mindfulness in Cognitive Behavior Therapy: Understanding and Applying the New Therapies, 132-163.

Sauer, S., Walach, H., Schmidt, S., Hinterberger, T., Lynch, S., Büssing, A., \& Kohls, N. (2013). Assessment of mindfulness: Review on state of the art. Mindfulness, $4(1), 3-17$.

Segal, Z. V., Williams, J. M. G., \& Teasdale, J. D. (2002). Mindfulness-based cognitive therapy for depression: A new approach to relapse prevention. New York: Guilford Press.

Shand, L. K., Brooker, J. E., Burney, S., Fletcher, J., \& Ricciardelli, L. A. (2014). Symptoms of posttraumatic stress in Australian women with ovarian cancer. Psycho-Oncology. Retrieved from http://onlinelibrary.wiley.com/doi/10.1002/pon.3627/full

Shand, L. K., Cowlishaw, S., Brooker, J. E., Burney, S., \& Ricciardelli, L. A. (2014). Correlates of post-traumatic stress symptoms and growth in cancer patients: a systematic review and meta-analysis. Psycho-Oncology. Retrieved from http://onlinelibrary.wiley.com/doi/10.1002/pon.3719/full

Shapiro, S. L., Carlson, L. E., Astin, J. A., \& Freedman, B. (2006). Mechanisms of mindfulness. Journal of Clinical Psychology, 62(3), 373-386.

Shennan, C., Payne, S., \& Fenlon, D. (2011). What is the evidence for the use of mindfulness-based interventions in cancer care? A review. Psycho-Oncology, 20(7), 681-697. 
Smith, B. W., Ortiz, J. A., Steffen, L. E., Tooley, E. M., Wiggins, K. T., Yeater, E. A., ... Bernard, M. L. (2011). Mindfulness is associated with fewer PTSD symptoms, depressive symptoms, physical symptoms, and alcohol problems in urban firefighters. Journal of Consulting and Clinical Psychology, 79(5), 613.

Smith, S. K., Zimmerman, S., Williams, C. S., \& Zebrack, B. J. (2009). Health status and quality of life among non-Hodgkin lymphoma survivors. Cancer, 115(14), $3312-3323$.

Sprangers, M. A., Tempelaar, R., van den Heuvel, W. J., de Haes, M., \& Hanneke, C. J. (2002). Explaining quality of life with crisis theory. Psycho-Oncology, 11(5), $419-426$.

Stafford, L., Foley, E., Judd, F., Gibson, P., Kiropoulos, L., \& Couper, J. (2013a). Mindfulness-based cognitive group therapy for women with breast and gynecologic cancer: a pilot study to determine effectiveness and feasibility. Supportive Care in Cancer, 21(11), 3009-3019.

Stafford, L., Foley, E., Judd, F., Gibson, P., Kiropoulos, L., \& Couper, J. (2013b). Mindfulness-based cognitive group therapy for women with breast and gynecologic cancer: a pilot study to determine effectiveness and feasibility. Supportive Care in Cancer, 21(11), 3009-3019.

Tamagawa, R., Giese-Davis, J., Speca, M., Doll, R., Stephen, J., \& Carlson, L. E. (2013). Trait Mindfulness, Repression, Suppression, and Self-Reported Mood and Stress Symptoms Among Women With Breast Cancer. Journal of Clinical Psychology, 69(3), 264-277. 
Tangney, J. P., Miller, R. S., Flicker, L., \& Barlow, D. H. (1996). Are shame, guilt, and embarrassment distinct emotions? Journal of Personality and Social Psychology, 70(6), 1256.

Teasdale, J. D., Moore, R. G., Hayhurst, H., Pope, M., Williams, S., \& Segal, Z. V. (2002). Metacognitive awareness and prevention of relapse in depression: empirical evidence. Journal of Consulting and Clinical Psychology, 70(2), 275.

Thompson, B. L., \& Waltz, J. (2008). Self-compassion and PTSD symptom severity. Journal of Traumatic Stress, 21(6), 556-558.

Thompson, B. L., \& Waltz, J. (2010). Mindfulness and experiential avoidance as predictors of posttraumatic stress disorder avoidance symptom severity. Journal of Anxiety Disorders, 24(4), 409-415.

Thompson, R. W., Arnkoff, D. B., \& Glass, C. R. (2011a). Conceptualizing Mindfulness and Acceptance as Components of Psychological Resilience to Trauma. Trauma, Violence, \& Abuse, 12(4), 220-235. http://doi.org/10.1177/1524838011416375

Thompson, R. W., Arnkoff, D. B., \& Glass, C. R. (2011b). Conceptualizing Mindfulness and Acceptance as Components of Psychological Resilience to Trauma. Trauma, Violence, \& Abuse, 12(4), 220-235. http://doi.org/10.1177/1524838011416375

Thompson, S., Eccleston, L., \& Hickish, T. (2011). Post-Traumatic Stress Disorder in Cancer Survivors: Recognising and Acknowledging the Symptoms. Retrieved from https://www.webmedcentral.com/article_view/2062 
Van Dam, N. T., Earleywine, M., \& Borders, A. (2010). Measuring mindfulness? An item response theory analysis of the Mindful Attention Awareness Scale. Personality and Individual Differences, 49(7), 805-810.

Vasterling, J. J., Duke, L. M., Brailey, K., Constans, J. I., Allain, A. N., \& Sutker, P. B. (2002). Attention, learning, and memory performances and intellectual resources in Vietnam veterans: PTSD and no disorder comparisons. Neuropsychology, 16(1), 5.

Vujanovic, A. A., Youngwirth, N. E., Johnson, K. A., \& Zvolensky, M. J. (2009). Mindfulness-based acceptance and posttraumatic stress symptoms among trauma-exposed adults without axis I psychopathology. Journal of Anxiety Disorders, 23(2), 297-303.

Wachen, J. S., Patidar, S. M., Mulligan, E. A., Naik, A. D., \& Moye, J. (2014). Cancerrelated PTSD symptoms in a veteran sample: association with age, combat PTSD, and quality of life. Psycho-Oncology. Retrieved from http://onlinelibrary.wiley.com/doi/10.1002/pon.3494/full

Watts, B. V., Schnurr, P. P., Mayo, L., Young-Xu, Y., Weeks, W. B., \& Friedman, M. J. (2013). Meta-analysis of the efficacy of treatments for posttraumatic stress disorder. The Journal of Clinical Psychiatry, (74), e541-e550.

Weinstein, N., Brown, K. W., \& Ryan, R. M. (2009). A multi-method examination of the effects of mindfulness on stress attribution, coping, and emotional wellbeing. Journal of Research in Personality, 43(3), 374-385.

Wilson, I. B., \& Cleary, P. D. (1995). Linking clinical variables with health-related quality of life: a conceptual model of patient outcomes. Jama, 273(1), 59-65. 
Wilson, J. P., Droždek, B., \& Turkovic, S. (2006). Posttraumatic shame and guilt. Trauma, Violence, \& Abuse, 7(2), 122-141.

Wilson, J. P., \& Keane, T. M. (2004). Assessing psychological trauma and PTSD. Guilford press. Retrieved from http://psycnet.apa.org/psycinfo/200421033-000

Wilson, J. P., SMITH, W., \& Johnson, S. K. (2013). A Comparative Analysis of PTSD Among Various. Trauma and Its Wake, 1, 142.

Witek-Janusek, L., Albuquerque, K., Chroniak, K. R., Chroniak, C., Durazo-Arvizu, R., \& Mathews, H. L. (2008a). Effect of mindfulness based stress reduction on immune function, quality of life and coping in women newly diagnosed with early stage breast cancer. Brain, Behavior, and Immunity, 22(6), 969-981.

Witek-Janusek, L., Albuquerque, K., Chroniak, K. R., Chroniak, C., Durazo-Arvizu, R., \& Mathews, H. L. (2008b). Effect of mindfulness based stress reduction on immune function, quality of life and coping in women newly diagnosed with early stage breast cancer. Brain, Behavior, and Immunity, 22(6), 969-981.

Wong, W. S., \& Fielding, R. (2007). Change in quality of life in Chinese women with breast cancer: changes in psychological distress as a predictor. Supportive Care in Cancer, 15(11), 1223-1230.

Würtzen, H., Dalton, S. O., Elsass, P., Sumbundu, A. D., Steding-Jensen, M., Karlsen, R. V., ... Johansen, C. (2013). Mindfulness significantly reduces self-reported levels of anxiety and depression: results of a randomised controlled trial among 336 Danish women treated for stage I-III breast cancer. European Journal of Cancer, 49(6), 1365-1373. 


\section{Education}

\begin{tabular}{|c|c|}
\hline 2016 - Present & $\begin{array}{l}\text { VA North Texas Health Care System - Dallas, Texas } \\
\text { Psychology Intern }\end{array}$ \\
\hline 2012-Present & $\begin{array}{l}\text { University of Louisville - Louisville, Kentucky } \\
\text { Doctoral Candidate, Clinical Psychology } \\
\text { Doctoral Dissertation: (Defended) The Relationship Between } \\
\text { Trauma and Health-Related Quality of Life in Lung Cancer } \\
\text { Patients: The Potential Protective Role of Mindfulness. } \\
\text { Dissertation Chair: Sandra E. Sephton, Ph.D. } \\
\text { Co-Mentor: Paul G. Salmon, Ph.D. }\end{array}$ \\
\hline 2008-2012 & $\begin{array}{l}\text { University of Illinois at Chicago - Chicago, Illinois } \\
\text { Bachelor of Arts in Applied Psychology, Highest Distinction } \\
\text { Honors Thesis: Spirituality, Religious Coping and Life Satisfaction } \\
\text { in Adult Sexual Assault Survivors. } \\
\text { Thesis Chair: Sarah E. Ullman, Ph.D. }\end{array}$ \\
\hline
\end{tabular}

\section{Clinical Experience and Training}

\section{Predoctoral Internship Clinical Experience - VA North Texas Healthcare System}

\section{Diamond Team (Outpatient Mental Health Clinic)}

Anticipated April 2017-July 2017

Major Rotation: individual psychotherapy, group psychotherapy, diagnostic interviewing, psychological assessment, review of electronic medical records, one-on-one supervisions, psychological assessment interpretation, report writing, and treatment planning for outpatient adults will be included. Will have the opportunity to learn and implement Acceptance and Commitment Therapy for Depression and co-lead groups such as Men's Combat PTSD Group, Women's Support/Trauma Group and mixed-gender DBT Skills Group.

Supervisor: Gloria Emmett, Ph.D., ABPP Clinical Psychology

\section{Community Living Center}

Anticipated January 2017-April 2017

Major Rotation: Inpatient comprehensive care unit for geriatric patients that includes acute rehabilitation, long-term dementia care wing and hospice palliative care. 
Opportunities for individual psychotherapy, supportive group psychotherapy, diagnostic interviewing, neuropsychological assessment, review of electronic medical records, oneon-one supervisions, neuropsychological assessment interpretation, report writing, and treatment planning for inpatient older adults are included. Wil represent psychology as part of an interdisciplinary team and consult with other medical providers to address patient care needs. Will develop competency in working with older adults in an acute rehabilitation setting, hospice and palliative care setting and long-term dementia patients. Supervisor: Helen Chung, Ph.D.

\section{Evidence Based Psychotherapy Rotation: Cognitive Processing Therapy Anticipated January 2017-July 2017}

Six-Month Rotation: will receive one-on-one training and supervision in Cognitive Processing Therapy. Will provide Cognitive Processing Therapy on an outpatient basis with adults with PTSD. Individual psychotherapy, diagnostic interviewing, review of electronic medical records, suicide risk assessment, safety planning, audio recording and clinical writing are included.

Supervisor: Anushka Pai, Ph.D.

\section{Gold Team (Inpatient Substance Abuse Clinic)}

October 2016-January 2017

Major Rotation: individual psychotherapy, group psychotherapy, diagnostic interviewing, psychological assessment, review of electronic medical records, one-on-one supervisions, psychological assessment interpretation, report writing, and treatment planning for patients in the residential substance abuse treatment program are included. Co-led residential Seeking Safety Groups, Relapse Prevention and Coping Skills Groups and CoDependency Groups. Developed competency in working with patients recovering from substance abuse. Evidence based therapy practiced included Motivational Interviewing, Cognitive-Behavioral Therapy for Insomnia, and Prolonged Exposure Therapy. Supervisor: Colleen Richardson, Psy.D.

\section{Evidence Based Psychotherapy Rotation: Interpersonal Psychotherapy}

July 2016-January 2017

Six-Month Rotation: received one-on-one training and supervision on Interpersonal Psychotherapy. Provided Interpersonal Psychotherapy on an outpatient basis with older adults with diagnoses of Major Depressive Disorder and Persistent Depressive Disorder. Individual psychotherapy, diagnostic interviewing, review of electronic medical records, suicide risk assessment, safety planning, audio recording and clinical writing are included.

Supervisor: Quavandra Perry, Ph.D.

\section{Silver Team (Outpatient Older Adult Clinic)}

July 2016-October 2016

Major Rotation: individual psychotherapy, group psychotherapy, diagnostic interviewing, neuropsychological assessment, review of electronic medical records, one-on-one supervisions, neuropsychological assessment interpretation, report writing, and treatment planning for outpatient older adults are included. Co-led Imagery Rehearsal Training and 
Cognitive Stimulation Groups with supervisors and postdoctoral fellows. Represented psychology as part of an interdisciplinary team and consulted with other medical providers to address patient care needs. Developed competency in working with older adults in an outpatient setting. Evidence based therapy practiced included CognitiveBehavioral Therapy for Depression and Imagery Rehearsal Therapy.

Supervisor: Catherine Dodson, Ph.D.

Supervisor: Heejin Kim, Psy.D. ABPP Neuropsychology

\section{$\underline{\text { Doctoral Program Clinical Training }}$}

\section{United States Army Recruiting Command (USAREC)}

July 2015-May 2016

Clinical Practicum: Clinical assessments of Soldiers selected for recruiting duty, review of electronic medical records, consultation with medical providers, psychological assessment interpretation, report writing, reading, and one-on-one supervision are included. Also assisted with teaching resilience and psychoeducation courses, and oneon-one coaching for Soldiers. Developed competency working with active service men and women and organizational/industrial psychology.

Supervisor: MAJ Chaska Gomez, Psy.D.

\section{University of Louisville Hospital}

April 2015-May 2016

Clinical Practicum: Neuropsychology practicum learning how to conduct dementia assessments. Clinical interviewing, administration of neuropsychological assessment battery, collateral interviewing, one-on-one supervision, chart review/assessment report writing, individual therapy, and reading are all activities that are included.

Supervisor: Elizabeth Cash, Ph.D.

\section{Athena's Sisters - Women's Military Group}

May 2015-May 2016

Clinical Practicum: Conducted brief psychological and behavioral assessments for military women with the goal of facilitating community referrals as needed.

Administration of brief assessment battery, interviewing, and supportive counseling are included. Developed competency working with active-duty and veteran women.

Supervisor: Bernadette Walter, Ph.D.

\section{The Office of David L. Winsch, Ph.D.}

February 2015-July 2015

Clinical Practicum: Conducted forensic neuropsychological assessment batteries for adults and children seeking social security benefits. Weekly individual supervision, neuropsychological assessments and scoring, chart review, and clinical report writing are included.

Supervisor: David Winsch, Ph.D.

\section{University of Louisville Psychological Services Practicum}

August 2014-June 2016 
Clinical practicum: Cognitive-Behavioral Therapy with adult clients. Weekly team supervision, peer consultation, one-on-one supervision, individual therapy, case conceptualization, audio/digital recording review, and chart review/clinical report writing activities are included.

Supervisor: Janet Woodruff-Borden, Ph.D.

\section{University of Louisville Psychological Services Center}

July 2014-June 2016

Clinic Assistant

Graduate clinic assistant at the Noble H. Kelley Psychological Services Center, an outpatient clinic at the University of Louisville. Responsibilities include opening and closing the clinic, phone intakes for therapy and assessment clients, crisis management, clinical interviews/intakes with therapy clients, supervision of graduate students, one-onone supervision, chart review/assessment report writing, and audio/visual digital recording review.

Supervisor: Bernadette Walter, Ph.D.

\section{University of Louisville Psychological Services Center Assessment Practicum} August 2013-June 2016 Clinical Practicum: Conducted neuropsychological test batteries for adults. Clinical interviewing, administration of standardized testing, one-on-one supervision and chart review/integrated clinical report writing are included.

Supervisor: David Winsch, Ph.D.

\section{University of Louisville Psychological Services Center Assessment Practicum} May 2013-June 2016

Clinical practicum: Conducted advanced placement testing and assessments for children. Clinical interviewing of parents/legal guardians, administration of standardized testing, one-on-one supervision, and chart review/clinical report writing are included. Supervisor: Bernadette Walter, Ph.D.

\section{Fort Knox Department of Behavioral Health}

August 2013-April 2014

Clinical practicum: Used integrative and empirically supported treatments for an active military population. Developed competence in working with active-duty servicemen and women. Collaborated on fitness-for-duty evaluations and psychological autopsies. Received weekly supervision and participated in peer supervision, chart review and clinical writing. Supervisor: Charles Thomas, Psy.D.

\section{University of Louisville Pain Management Clinic} May 2013-August 2013

Clinical practicum: Brief supportive therapy and assessment in a hospital setting to patients with chronic pain. Weekly individual supervision, peer consultation, peersupervision, chart review/clinical report writing, and individual therapy utilizing mindfulness techniques are included. 
Supervisor: Brian Monsma, Ph.D.

\section{University of Louisville Psychological Services Practicum}

August 2012-August 2014

Clinical practicum: Mindfulness-based psychotherapy with adult clients. Weekly team supervision, peer consultation, one-on-one supervision, individual therapy, case conceptualizations, audio/digital recording review, and chart review/clinical report writing activities are included.

Supervisor: Paul Salmon, Ph.D.

\section{Publications}

Rebholz, W.N.; Cash, E.; Zimmaro, L.A.; Bayley-Veloso, R.; Phillips, K.; Siwik, C; Chagpar, A.B.; Dhabhar, F.S.; Spiegel, D.; Saltsman Bell, B.N.; Sephton, S.E. (In Press). Distress and QOL in an Ethnically Diverse Sample Awaiting Breast Cancer Surgery. Journal of Health Psychology.

Zimmaro, L.A., Salmon, P., Naidu, H., Rowe, J., Phillips, K., Rebholz, W. N., GieseDavis, J., Cash, E., Dreeben, S., Bayley-Veloso, R., Jablonski, M., Hicks, A., Siwik, C., Sephton, S.E. (2016). Association of Dispositional Mindfulness with Stress, Cortisol, and Well-being Among University Undergraduate Students. Mindfulness. 1-12.

Bayley-Veloso, R. \& Salmon, P.G. (2016) Yoga in clinical practice. Mindfulness, 7(2), 308-319.

Cash, E., Salmon, P., Weissbecker, I., Rebholz, W. N., Bayley-Veloso, R., Zimmaro, L. A., Floyd, A., Dedert, E. \& Sephton, S. E. (2014). Mindfulness meditation alleviates fibromyalgia symptoms in women: Results of a randomized clinical trial. Annals of Behavioral Medicine, 49(3), 319-330.

\section{Published Abstracts and Presentations}

Phillips, K., Bayley-Veloso, R., Zimmaro, L.A., Siwik, C., Hicks, A.M., Cash, E., Salmon, P., \& Sephton, S.E. (2016). Does Living Situation Affect Stress and Health Outcomes Among Cancer Patients? Poster presentation at the American Psychosomatic Society 74th Annual Scientific Conference, Denver, CO.

Siwik, C.J., Phillips, K., Zimmaro, L.A., Bayley-Veloso, R., Hicks, A., Cash, E., Salmon, P., Sephton, S.E. (2016). Psychological and Physiological Effects of Problem-focused and Emotional Approach to Coping Styles in Gynecological Cancer Patients. Poster presentation at the American Psychosomatic Society 74th Annual Scientific Conference, Denver, CO.

Hicks, A., Salmon, P., Phillips, K., Zimmaro, L., Siwik, C., Bayley-Veloso, R., Albert, C., Fields, O., Cash, E., \& Sephton, S.E. (2016) The Role of Mindfulness in Stress and Depressive Symptoms of Undergraduate Students. Poster presentation at the American Psychosomatic Society 74th Annual Scientific Conference, Denver, CO. 
Bayley-Veloso, R., Weissbecker, I., Rebholz, W.N., Cash, E., Zimmaro, L.A., Phillips, K., Salmon, P., Sephton, S.E. (2015) Do Traumatic Events Build Resilience and Promote Survival Through Social Support in Gynecologic Cancer?. Poster presentation at the American Psychosomatic Society 73rd Annual Scientific Conference, Savannah, GA.

Cash, E.; Chilton, P.; Rebholz, W.; Bayley-Veloso, R.; Zimmaro, L.; Chagpar, A.B.; Spiegel, D.; Dhabhar, F.S.; Sephton, S.E. (2015). HPA and Rest/Activity Rhythms Independently Associated with Different Aspects of Inflammatory Response in Patients Awaiting Treatment for Breast Cancer. Oral presentation at the American Psychosomatic Society 73rd Annual Scientific Meeting, Savannah, GA, March 18-21.

Rebholz, W.N., Weissbecker, I., Cash, E., Bayley-Veloso, R., Zimmaro, L.A., Phillips, K., Sephton, S.E. (2015). Diurnal Cortisol Rhythms and Systemic Norepinephrine Predict Gynecologic Cancer Survival. Poster presentation at the American Psychosomatic Society 73rd Annual Scientific Conference, Savannah, GA.

Zimmaro, L.A., Rebholz, W.N., Cash, E., Bayley-Veloso, R., Phillips, K., Salmon, P., Sephton, S.E. (2015). Optimism as a Moderator of Psychoneuroimmune Pathways in Lung Cancer Patients. Poster presentation at the American Psychosomatic Society 73rd Annual Scientific Conference, Savannah, GA.

Phillips, K., Salmon, P., Patel, H., Rowe, J., Rebholz, W.N., Zimmaro, L.A., BayleyVeloso, R., Cash, E., Giese-Davis, J., Sonnier, H., Sephton, S.E. (2015). The Role of Mindfulness in Stress and Health Outcomes in University and Undergraduate Students. Poster presentation at the American Psychosomatic Society 73rd Annual Scientific Conference, Savannah, GA.

Bayley, R.C., Weissbecker, I., Rebholz, W.N., Cash, E., Zimmaro, L.A., Salmon, P., Sephton, S.E. (2014). Recent Traumatic Events May Complicate Adjustment to Gynecologic Cancer. Poster presented at the American Psychosomatic Society 72nd Annual Scientific Conference, San Francisco, CA.

Rebholz, W.N., Weissbecker, I., Cash, E., Bayley, R., Zimmaro, L.A., Sephton, S.E. (2014). Distress and Support: Links with Circadian Disruption and Quality of Life in Gynecologic Cancer. Talk was given at the American Psychosomatic Society 72nd Annual Scientific Conference, San Francisco, CA.

Sephton, S.E., Cash, E., Chagpar, A., Spiegel, D., Rebholz, W.N., Bayley, R.C., Zimmaro, L.A., and Dhabhar, F.S. (2014). Biological Correlates of Marital Status in Recently Diagnosed Breast Cancer Patients. Talk was given at the American Psychosomatic Society 72nd Annual Scientific Conference, San Francisco, CA.

Zimmaro, L.A., Cash, E., Dedert, E., Rebholz, W.N., Bayley, R.C., Salmon, P., Sephton, S.E. (2014). Distress, Coping and Support in Lung Cancer: Gender Differences and 
Associations with Psychopathology. Poster presented at the American Psychosomatic Society 72nd Annual Scientific Conference, San Francisco, CA.

Bayley, R.C., Rebholz, W.N., Salmon, P., Sephton, S.E. (2013). Perceived Distress, Coping and Health Behaviors in Lung Cancer Patients. Poster presented at the American Psychosomatic Society $71^{\text {st }}$ Annual Scientific Conference, Miami, FL.

\section{Manuscripts in Progress}

Bayley-Veloso, R., Szabo, Y., Ellsworth, M., Sephton, S.E., \& Salmon, P.G. The role of self-compassion following traumatic stress exposure.

Vines, L.M., Bayley-Veloso, R. \& Salmon, P. Embrace the suck: Creating receptivity to mindfulness-based interventions within military culture.

\section{Programs, and Workshops}

2015.05.11. Bayley-Veloso, R. \& Szabo, Y. Trauma Processing and Courageous Next

Steps. Half-day workshop as part of the Sisters Healing Sisters Retreat with Athena's Sisters Women's Military Group. Life Adventure Center, Versailles, Ky.

2015.04.11. Bayley-Veloso, R., Szabo, Y., Altman, J., McDonough, S. Identity and SelfCompassion for Veterans. Workshop for Athena's Sisters Women's Military Group.

2015.11.16 Fleagle, L., Bayley-Veloso, R., Cook, J. CBT for the Medically Ill. Grand Rounds Presentation for the University of Louisville Psychiatry Residency Program.

2015.08.19 Salmon, P.G., Bayley-Veloso, R., Warnecke, A. Stress, College and Mindfulness. Workshop for the University of Louisville Cardinal Covenant, College of Arts and Sciences.

2015.05.11 Bayley-Veloso, R., Richards, A., Knight, H., Rebholz, W.N., Szabo, Y. SelfEmpowerment and Self-Compassion for Veterans. Workshop for Athena's Sisters Women's Military Group.

2015.01.30 Salmon, P.G., Bayley-Veloso, R. Introduction to Mindfulness. Workshop for the University of Louisville Cardinal Covenant, College of Arts and Sciences.

2014.11.01 Salmon, P.G., Altman, J., Bayley-Veloso, R., Cash, E., Ellsworth, M., Phillips, K., Rebholz, W., Sephton, S., and Zimmaro, L. A Day of Mindfulness, Workshop for the University of Louisville Alumni Association, College of Arts and Sciences.

2013.01.24 Bayley, R. Principles and Applications of Mindfulness in Health and Mindful Movement, Workshop for the Department of Psychology, Bellarmine University.

2013.06.13 Bayley, R. Principals and Applications of Mindfulness for Youth. Workshop for the Center of Mental Health Disparities, University of Louisville. 
Research Experience

\section{Project Coordinator: Effects of Stress in Ovarian Cancer Outcomes}

June 2015-June 2016

Manage regulatory issues through the Internal Review board for this study on the effects of stress on cancer outcomes. Studied mechanisms that predict survival and well-being for patients with cancer.

Directed by: Sandra Sephton, Ph.D.

\section{Project Coordinator: Mindfulness-Based Stress Reduction for Parkinson's Disease Patients and Caregivers}

October 2013-June 2016

Manage regulatory issues through the Internal Review Board for this study on the effects of a Mindfulness-Based Stress Reduction program on health related quality of life for patients and caregivers.

Directed by: Paul Salmon, Ph.D.

\section{Graduate Research Assistant: Understanding the Prognostic Significance of Circadian Disruption in Lung Cancer and Piloting an Intervention}

January 2013-Present

Co-wrote a $\$ 150,000$ grant that was funded by the Kentucky Lung Cancer Research

Board. Studied the mechanisms by which psychosocial factors affect tumor progression and ameliorating stress-illness effects with a mindfulness-based intervention. Assisted with study design, created the database and syntax for analyses, data collection, preparing subject materials and IRB compliance. Mentored a team of undergraduate students in charge of data entry and working on undergraduate honors theses. Dissertation data was utilized from this sample.

Directed by: Sandra Sephton, Ph.D.

\section{Graduate Research Assistant: Mindfulness and Working Memory Capacity in the Context of Acute Stress}

August 2012-June 2015

Researched background information on the International Affective Picture System, and the association between Working Memory Capacity and Mindfulness. Assisted with filing Institutional Review Board Paperwork, data collection, preparing subject materials and IRB compliance. Interviewed, selected and mentored undergraduate research assistants. Analyzed data and assisted on projects for publication.

Directed by: Paul Salmon, Ph.D.

Dissertation by: Lauren Vines, Ph.D.

\section{Graduate Research Assistant: iPod-Based Coping Skills for Newly Diagnosed Breast Cancer Patients}

July 2012-August 2013

Studied the effectiveness of iPod-based coping skills intervention. Co-created and organized a mindfulness-based drop-in group for participants. Taught participants

mindfulness-based breathing exercises, gentle yoga and how to incorporate mindfulness 
in their everyday lives while coping with cancer. Created a Mindfulness-Group advertisement to be distributed to the clinics.

Directed by: Sandra Sephton, Ph.D.

\section{Professional Membership and Credentials}

American Psychological Association

Present

American Psychosomatic Society

Psi Chi International Honor Society in Psychology

Certified Yoga Instructor (Yoga Alliance RYT/200 level)

Association for Psychological Science

2014

\section{Teaching Experience}

\section{Graduate Teaching Assistant}

University of Louisville Department of Psychological and Brain Sciences

July 2014 - May 2016

Courses Assisted: Psychology 693: Interviewing Skills Practicum; Psychology 680:

Cognitive Assessment

\section{Professional Development}

\section{Safe Zone Training}

University of Louisville LGBTQ Center

October 28, 2015 and November 4, 2015

Attended a two-day training on the LGBTQ community. Learned common terminology, how to be an effective ally and how to provide a safe space for LGBTQ clients.

\section{Operation Immersion}

Wendall H. Ford Regional Training Center, Kentucky Army National Guard May 19, 2015 - May 22, 2015

Attended a four-day intensive training on military culture for civilians in mental health. Key themes of training included basic training, mobilization, deployment and demobilization. Attended seminars with mental health professionals, which focused on introduction to military culture, trauma informed care, combat/deployment stress, and suicide prevention using ACE protocol. Participated in physical fitness training, field training, combat simulations, and urban warfare exercises under the direction of soldiers in the Kentucky Army National Guard.

\section{Honors and Awards}

\begin{tabular}{lll}
\hline 2016 Spring & $\begin{array}{l}\text { Department of the Army Certificate of Appreciation for Exemplary } \\
\text { Service and Excellent Leadership. } \\
2015 \text { Spring }\end{array}$ \\
& $\begin{array}{l}\text { Research recognized at the American Psychosomatic Society } 73^{\text {rd }} \\
\text { Annual Conference by the Program Committee as being a topic of } \\
\text { clinical interest and featured in a narrated poster tour }\end{array}$ \\
$2012-2014$ & $\begin{array}{l}\text { University of Louisville Graduate Student Fellowship Award } \\
2012 \text { Spring }\end{array} \quad$ UIC Chancellor's Student Service and Leadership Award
\end{tabular}




\section{References}

Catherine Dodson, Ph.D.

VA North Texas Healthcare System, Dallas, TX

Supervisory Psychologist

Email: Mary.Dodson2@va.gov

Heejin Kim, Psy.D., ABBP Neuropsychology

VA North Texas Healthcare System, Dallas TX

Supervisory Neuropsychologist

Email: Heejin.Kim@va.gov

Elizabeth Cash, Ph.D.

University of Louisville School of Medicine, Louisville, KY

Supervisory Psychologist

Phone: (502) 561-7268

Email: liz.cash@louisville.edu

Sandra E. Sephton, Ph.D.

University of Louisville, Louisville, KY

Dissertation Chair and Co-Mentor

Phone: (502) 852-1166

Email: sephton@louisville.edu 